\title{
Some Bayesian generalizations of the integer-valued autoregressive model
}

Helton Graziadei de Carvalho

\author{
DOCTORAL THESIS \\ PRESENTED TO THE \\ INSTITUTO DE \\ Matemática e Estatística \\ OF THE \\ UNIVERSIDADE OF SÃO PAULO \\ FOR ATTAINMENT OF \\ THE TITLE OF \\ Doutor EM CIÊNCIAS
}

Program: Statistics

Advisor: Prof. Dr. Hedibert Freitas Lopes

During the development of this work, the author received financial support from CAPES and FAPESP, Fundação de Amparo à Pesquisa do Estado de São Paulo, process number 2017/10096-6

São Paulo, March of 2020 


\section{Some Bayesian generalizations of the integer-valued autoregressive model}

This version of the thesis contains the corrections and modifications suggested by the Judging Commitee during the defense of the original version of this work, held on $17 / 02 / 2020$. A copy of the original version is available in the Institute of Mathematics and Statistics of the University of São Paulo.

Judging Committee:

- Prof. Dr. Hedibert Freitas Lopes (advisor) - Insper

- Prof. Dr. Rinaldo Artes - Insper

- Prof. Dr. Luís Gustavo Esteves - IME-USP

- Prof. Dr. Rafael Izbicki - UFSCar

- Prof. Dr. Ricardo Sandes Ehlers - ICMC-USP 
The opinions, hypotheses, conclusions and recommendations expressed in this material are the responsibility of the authors and do not necessarily reflect the views of FAPESP. 


\section{Acknowledgements}

This thesis would not be possible without the guidance of my supervisor Professor Hedibert Lopes. I am thankful for his patient guidance, encouragement and advice he has provided throughout my doctorate. I am also extremely grateful to Professor Paulo Marques for his incommensurate support and advice.

As a visiting student at Bocconi University, I had the privilege to be hosted by Professor Igor Pruenster which gave me all the necessary support during my one-year period in Milan. Our discussions about Bayesian nonparametrics, statistics and life will be forever in my memory. Also, I thank Professor Antonio Lijoi for his interest and support on my research.

My friends from the Departament of Statistics of IME-USP had a substantial role during the whole period of the doctorate program, especially those always available for discussions with beer. To Luisa Gonçalves for all her love, patience and care. Ultimately, I thank my parents for their love but also for giving me strength to chase my dreams and always support me to move on. To my brother, for all his help and to always believe in myself.

I thank CAPES and FAPESP, Fundação de Amparo à Pesquisa do Estado de São Paulo, process number 2017/10096-6, for the financial support. 


\section{Abstract}

CARVALHO, H. G. Some Bayesian generalizations of the integer-valued autoregressive model. 2020. 65 p. Tese (Doutorado) - Instituto de Matemática e Estatística, Universidade de São Paulo, São Paulo, 2020.

In this thesis, we develop Bayesian generalized models for analyzing time series of counts. In our first proposal, we use a finite mixture to define the marginal distribution of the innovation process, in order to potentially account for overdispersion in the time series. Our second contribution uses a Dirichlet process at the distribution of the time-varying innovation rates, which are softly clustered through time. Finally, we examine issues of prior sensitivity in a semi-parametric extended model in which the distribution of the innovation rates follows a Pitman-Yor process. A graphical criterion to choose the Pitman-Yor base measure hyperparameters is proposed, showing explicitly that the Pitman-Yor discount parameter and the concentration parameter can interact with the chosen base measure to yield robust inferential results. The posterior distribution of the models parameters is obtained through data-augmentation schemes which allows us to obtain tractable full conditional distributions. The prediction performance of the proposed models are put to test in the analysis of two real data sets, with favorable results.

Keywords: INAR(1), Finite mixture, Dirichlet process, Pitman-Yor process. 


\section{Resumo}

CARVALHO, H. G. Algumas generalizações bayesianas do modelo autorregressivo de valores inteiros.. 2020. 65 f. Tese (Doutorado) - Instituto de Matemática e Estatística, Universidade de São Paulo, São Paulo, 2020.

Nesta tese, desenvolvemos generalizações bayesianas para analisar séries temporais de contagem. Primeiramente, modelamos a distribuição marginal do processo de inovação através de um modelo de mistura finita, de modo a acomodar sobredispersão na série temporal. Em nossa segunda contribuição, utilizamos um processo Dirichlet na distribuição das taxas de inovação, que são clusterizadas temporalmente. Finalmente, exploramos questões de sensibilidade da distribuição a priori em um terceiro modelo em que a distribuição das taxas de inovação segue um processo de Pitman-Yor. Propomos um critério gráfico para escolher os hiperparâmetros da medida base do process, mostrando explicitamente que o parâmetro de desconto e o parâmetro de concentração podem interagera com a medida base escolhida para produzir resultados inferenciais robustos. As distribuições a posterior dos parâmetros dos modelos são obtidas por meio da técnica de dados aumentados, o que viabiliza a obtenção de distribuições condicionais completas facilmente tratáveis. A performance preditiva são avaliadas em dois conjuntos de dados reais, com resultados favoráveis.

Palavras-chave: INAR(1), Misturas finitas, Processo Dirichlet Processo de Pitman-Yor. 


\section{Contents}

List of Abbreviations $\quad$ xi

List of Figures $\quad$ xiii

List of Tables $\quad$ Xv

1 Introduction $\quad 1$

1.1 Contributions . . . . . . . . . . . . . . . . . . 2

1.2 Organization of the Thesis . . . . . . . . . . . . . . 3

2 A Bayesian integer-valued autoregressive model with adaptive overdispersion 5

2.1 Adaptive INAR(1) model . . . . . . . . . . . . . . . . . 5

2.2 Data augmentation . . . . . . . . . . . . . . . . . 8

2.3 Full conditional distributions . . . . . . . . . . . . . . . . . . . 9

2.4 Bayesian forecasting . . . . . . . . . . . . . . . . 11

2.5 Simulation Study . . . . . . . . . . . . . . . . . . . . . . 12

3 Semiparametric modeling of INAR processes $\quad 15$

3.1 Generalized INAR(1) model . . . . . . . . . . . . . . . . 15

3.2 Data augmentation . . . . . . . . . . . . . . . . . . . 16

3.3 The Dirichlet process . . . . . . . . . . . . . . . . . . . . . . . 18

3.4 DP-INAR(1) model . . . . . . . . . . . . . . . . . 19

3.5 Choice of prior parameters . . . . . . . . . . . . . . 23

3.6 Bayesian forecasting . . . . . . . . . . . . . . . . . . . 24

3.7 Burglary data . . . . . . . . . . . . . . . . . . . 27

4 A higher order semi-parametric model for time series of counts 37

4.1 Generalizing the autoregressive order . . . . . . . . . . . . . 37

4.2 Pitman-Yor process . . . . . . . . . . . . . . . . . 40

$4.3 \quad \mathrm{PY}-\operatorname{INAR}(p)$ model . . . . . . . . . . . . . . . . . . 41

4.4 Prior sensitivity . . . . . . . . . . . . . . . . . . . . . 44

4.5 Bayesian forecasting . . . . . . . . . . . . . . . . 51 
4.6 Earthquake count data . . . . . . . . . . . . . . . . . 52

$\begin{array}{lll}5 & \text { Conclusions } & 57\end{array}$

5.1 Final considerations . . . . . . . . . . . . . . . . . . 57

5.2 Suggestions for future research . . . . . . . . . . . . . . . 58

A BayesINAR: An $R$ package for Bayesian modeling time series of counts 59

$\begin{array}{ll}\text { References } & 63\end{array}$ 


\section{List of Abbreviations}

INAR Integer-valued autoregressive model

DP Dirichlet Process

PY Pitman-Yor process

iid independent and identically distributed 


\section{List of Figures}

2.1 Simulated time series from the AdINAR(1) model, exhibiting the behavior of the model for two extreme values of the mixture weight parameter $w$. . . . 7

2.2 The data augmented AdINAR(1) model. . . . . . . . . . . . . . . . . 10

3.1 The data augmented DP-INAR(1) model. . . . . . . . . . . . . . . . . . 21

3.2 Cross-validation scheme for one-step-ahead predictions. For each line, the black dots indicate the training set. Predictions are made for the target epoch marked with an $\times \ldots \ldots \ldots \ldots \ldots$. . . . . . . . . . . 26

3.3 Monthly burglary events for patrol area 58. . . . . . . . . . . . . . 27

3.4 Marginal posterior distributions of the $\operatorname{AdINAR}(1)$ model parameters $\alpha, \theta, \lambda$, and $w$ for patrol area 58. . . . . . . . . . . . . . . . . . . . . . 29

3.5 Contour plot of the Kullback-Leibler divergence associated with the optimization of the base measure hyperparameters for patrol area 58. . . . . . . . .

3.6 Marginal posterior distributions of the DP-INAR(1) model parameters $\alpha, \lambda_{3}$, $\lambda_{18}$, and $\lambda_{96}$ for patrol area 58. . . . . . . . . . . . . . . . 31

3.7 DP-INAR(1) model prior and posterior distributions for the number of clusters $K$, in gray and black respectively, for patrol area 58. . . . . . . . . . . .

3.8 Monthly burglary events for patrol area 58 (solid line) and the Bayes estimates of $\lambda_{t}, E\left(\lambda_{t} \mid y\right)$, (dashed line) under the DP-INAR(1) model. . . . . . . . .

3.9 Markov chains associated with the AdINAR(1) model marginal posterior distributions of parameters $\alpha, \theta, \lambda$, and $w$ for patrol area 58 . The gray rectangles indicate the burn-in periods. . . . . . . . . . . . . . . . .

3.10 Markov chains associated with the DP-INAR(1) model marginal posterior distributions of parameters $\alpha, \lambda_{3}, \lambda_{18}$, and $\lambda_{96}$ for patrol area 58. The gray rectangles indicate the burn-in periods. . . . . . . . . . .

4.1 Prior distributions of $K$ in the Pitman-Yor process, with $\sigma \in\{0,0.25,0.5,0.75\}$, $n=50$ and $\tau$ fixed such that $E(K)=25 \ldots \ldots \ldots \ldots$

4.2 Formation of the elbows for $\sigma=0.5$ (left) and $\sigma=0.75$ (right). The red dotted lines indicate the chosen values of $\lambda_{\max } \ldots \ldots \ldots$ 
4.3 Posterior distributions of the number of clusters $K$ for the simulated time series with $\sigma=0$ and $k_{0}=4,10,16,30$. The red dotted lines indicate the value of $k_{0} \ldots \ldots \ldots \ldots \ldots \ldots \ldots \ldots \ldots \ldots \ldots \ldots \ldots \ldots$

4.4 Posterior distributions of the number of clusters $K$ for the simulated time series with $\sigma=0.25$ and $k_{0}=4,10,16,30$. The red dotted lines indicate the value of $k_{0}$.

4.5 Posterior distributions of the number of clusters $K$ for the simulated time series with $\sigma=0.5$ and $k_{0}=4,10,16,30$. The red dotted lines indicate the value of $k_{0}$.

4.6 Posterior distributions of the number of clusters $K$ for the simulated time series with $\sigma=0.75$ and $k_{0}=4,10,16,30$. The red dotted lines indicate the value of $k_{0} \ldots \ldots \ldots \ldots \ldots \ldots \ldots$

4.7 Swallow tails obtained by multidimensional scaling. The left and right figures show the assigned and simulated cluster labels, respectively . . . . . . . . . . 51

4.8 Worldwide earthquake events from 1900 to 2018 (per year). . . . . . . . . . 53

4.9 Marginal posterior distributions of the PY-INAR(3) model parameters $\alpha_{1}, \alpha_{2}$ and $\alpha_{3}$ for the earthquake data set. . . . . . . . . . . . 53

4.10 Elbow for the earthquake data set with $\sigma=0.75$ and $k_{0}=30 \ldots \ldots$. . . . 54

4.11 Markov chains associated with the PY-INAR(3) model marginal posterior distributions of parameters $\alpha_{1}, \alpha_{2}, \alpha_{3}$ for the earthquake data set. The gray rectangles indicate the burn-in periods. . . . . . . . . . . . . . . . . . . . 54 


\section{List of Tables}

2.1 Means of the Monte Carlo errors for the 100 simulated time series from the $\operatorname{AdINAR}(1)$ with parameters for the $\left(\alpha_{0}, \theta_{0}, \lambda_{0}, w_{0}\right)=(0.10,0.15,5.66,0.9) \ldots 14$

2.2 Means of the Monte Carlo errors for the 100 simulated time series from the $\operatorname{AdINAR}(1)$ with parameters for the $\left(\alpha_{0}, \theta_{0}, \lambda_{0}, w_{0}\right)=(0.10,0.15,5.66,0.5)$.

2.3 Means of the Monte Carlo errors for the 100 simulated time series from the $\operatorname{AdINAR}(1)$ with parameters for the $\left(\alpha_{0}, \theta_{0}, \lambda_{0}, w_{0}\right)=(0.10,0.15,5.66,0.1)$.

3.1 Mean absolute deviations for the out-of-sample predictions of the $\operatorname{INAR}(1)$, AdINAR(1) and DP-INAR(1) models. The last two columns show the relative variations for the $\operatorname{AdINAR}(1)$ and DP-INAR(1) models with respect to the INAR(1) model (lower is better). For each patrol area, the best mean absolute deviation is written in bold face. . . . . . . . . . . . . . . . 36

4.1 Confusion matrix for the cluster assignments. . . . . . . . . . . . . . . 46

4.2 Out-of-sample MAE's for the $\operatorname{INAR}(p)$ and the PY-INAR $(p)$ models, with orders $p=1,2$, and 3 . The last column shows the relative variations of the MAE's for the PY-INAR $(p)$ models with respect to the corresponding MAE's for the $\operatorname{INAR}(p)$ models. . . . . . . . . . . . . . . . . 55 


\section{Chapter 1}

\section{Introduction}

Time series of counts arise in a multiplicity of phenomena, ranging from econometrics and finance to ecology and meteorology. The development of models for this kind of data has attracted significant attention over the past few years, mainly motivated by the first-order integer-valued autoregressive (INAR) model, proposed in the seminal papers of McKenzie [1985] and Al-Osh and Alzaid [1988]. This model has a simple structure, natural interpretation and advantages over the ordinary continuous autoregressive (AR) models, especially in "low" count scenarios in which the normal approximation may be imprecise.

Essentially, the INAR(1) model defines a time-homogeneous discrete Markovian process in which the counts at one epoch are a random sum of two components. The first component comprises a fraction of the counts at the previous epoch, which is called binomial thinning operator in the discrete time series literature [Weiß, 2018]. The main motivation for using an operator of this sort is that the usual $\operatorname{AR}(1)$ recursion, $Y_{t}=\phi Y_{t-1}+\epsilon_{t}$, cannot be applied to count time series, since the multiplication parcel does not necessarily preserve the discreteness of the counts. Also, the second component is the counts associated with an independent innovation process. In their first proposal, McKenzie [1985] and Al-Osh and Alzaid [1988] assumes that the innovations are independent and identically distributed Poisson random variables with constant innovation rate. However, a large body of research has been dedicated to modifications and generalizations of this model. For instance, Freeland [1998] develops higher order generalizations of the INAR(1) process. Weiß [2008] shows the thinning operators that are commonly used in time series of counts. Bourguignon and Weiß [2017] introduces generalized thinning operators to induce overdispersion, equidispersion and underdispersion in in the marginal distributions. Furthermore, Jazi et al. [2012] introduces a INAR(1) model with Geometric-distributed innovations. Under the Bayesian perspective,

Silva et al. [2005] and Silva et al. [2009] develops Metropolis-Hastings algorithms as a means 
to approximate the posterior distribution of the INAR(1) model parameters. For a concise review on the state-of-the-art autoregressive models for time series of counts, see Weiß [2018] and Davis et al. [2016].

\subsection{Contributions}

The main purpose of this thesis is to develop Bayesian generalizations of the INAR(1) model in order to improve predictive performance. In our first proposal, the adaptive integervalued autoregressive model (AdINAR(1) model hereafter) we make use of a finite mixture to define the marginal distributions of the innovation process. The motivation for this AdINAR(1) model is the possibility to account for overdispersed time series. Our second contribution is a hierarchical extension of the INAR(1) model, implemented with the help of a Dirichlet process [Ferguson, 1973] placed at the top of the model hierarchy. In this DPINAR(1) model, the innovation rates may vary through time, and in their modeling we benefit from the clustering properties of the Dirichlet process. In other words, this flexible model accommodates multimodal patterns on the innovation process, without previously specifying the number of modes. We show a proposition, by using moment generating functions, which allows us to obtain the predictive distribution of future steps of the process and, consequently, obtain pointwise predictions. In our third contribution, we extend the DP-INAR(1) for the higher-order autoregressive model, the INAR(p) model, with a finite Dirichlet prior on the autoregressive thinning vector. This prior is more general than the proposal of Neal and Kypraios [2015] since it accounts dependencies on the autoregressive parameters. In this hierarchy, we place a Pitman-Yor process on the innovation rates distribution. The sensitivity of the parameters is extensively investigated. In this context, we propose a graphical criterion to choose the hyperparameters of the base measure. Also, dataaugmentation schemes are explored in all models as a means to obtain an approximation of the posterior distribution of parameters, which are substantially simpler than the adaptive Metropolis-Hastings algorithm proposed in Silva et al. [2005]. We develop an R package, the BayesINAR, as a tool to learn the parameters of the proposed models as well as forecasting future steps of the discrete time series. 


\subsection{Organization of the Thesis}

In Chapter 2, we define the AdINAR(1) model, in order to analyze possibly overdispersed time series of counts. However, since parametric model can be inadequate to describe realistic datasets [Sudderth, 2006], we propose, in Chapter 3, the DP-INAR(1) model which places a Dirichlet Process at the top of hierarchy whose main feature is its capability to learn a latent pattern of heterogeneity in the distribution of the process innovation rates. We apply both models in a real time series of crimes in Pittsburgh in Section 3.7, showing that the proposed models outperform the INAR(1) model for most of patrol areas. Nevertheless, it has been highlighted that Dirichlet Processes heavily depends on the specification of the hyperparameters [Canale and Prünster, 2017, Dorazio, 2009]. As a consequence, in Chapter 4, we propose a higher order INAR model in which a Pitman-Yor process on the innovation rates distribution is placed at the top of the model hierarchy. Furthermore, we assess the forecasting performance of the models in two real data sets at the end of Chapters 3 and 4 . In Chapter 5, we discuss the main conclusions of this work with possible directions of future research. In the Appendix, we show some guidelines for using an $\mathrm{R}$ package with functions for training and prediction from the proposed models. 


\section{Chapter 2}

\section{A Bayesian integer-valued autoregressive model with adaptive overdispersion}

In this chapter, we generalize the Poisson integer-valued autoregressive model, specifying a Poisson-Geometric mixture distribution, with unknown mixture weight parameter, for the process innovations. This mixture distribution allows the model to account for overdispersion in a time series of counts. Full conditional distributions of the model parameters are derived in simple closed forms through a data augmentation scheme, allowing us to forecast future counts in a thoroughly probabilistic way.

\subsection{Adaptive INAR(1) model}

We define the Adaptive INAR(1) model (AdINAR(1) model for short) as a generalization of the original INAR(1) model introduced in Al-Osh and Alzaid [1988] and McKenzie [1985].

For an integer-valued time series $\left\{Y_{t}\right\}_{t \geq 1}$, let the innovations $\left\{Z_{t}\right\}_{t \geq 2}$, given parameters $0<\theta \leq 1, \lambda>0$ and $0 \leq w \leq 1$, be a sequence of conditionally independent and identically distributed $w \times \operatorname{Geometric}(\theta)+(1-w) \times \operatorname{Poisson}(\lambda)$ random variables. Given a parameter $0 \leq \alpha \leq 1$, let $\left\{B_{i}(t): i \geq 0, t \geq 2\right\}$ be a family of conditionally independent and identically distributed Bernoulli $(\alpha)$ random variables. Also, given all the parameters, we assume that the innovations $\left\{Z_{t}\right\}_{t \geq 2}$ and the family $\left\{B_{i}(t): i \geq 0, t \geq 2\right\}$ are conditionally independent. The AdINAR(1) model is defined by the same functional relation of the INAR(1) process [Al-Osh and Alzaid, 1988, Weiß, 2018]:

$$
\underbrace{Y_{t}}_{\text {Count at time } t}=\underbrace{\alpha \circ Y_{t-1}}_{\text {Survivors from } t-1}+\underbrace{Z_{t}}_{\text {Innovation at time } t}
$$


for $t \geq 2$, in which $\circ$ denotes the binomial thinning operator, defined by $\alpha \circ Y_{t-1}=$ $\sum_{i=1}^{Y_{t-1}} B_{i}(t)$, if $Y_{t-1}>0$, and $\alpha \circ Y_{t-1}=0$, if $Y_{t-1}=0$.

Informally, in terms of an epidemic analogy, at epoch $t$, the number of cases $Y_{t}$ is the sum of the new cases $Z_{t}$ and the cases that matured from the previous epoch $t-1$. Essentially, the binomial thinning operator removes a random number of the $Y_{t-1}$ cases that were present at the previous epoch. Due to the form of the mixture distribution of the innovations $Z_{t}$, if $w=0$ with probability one, we recover the original INAR(1) model with Poisson innovations.

Figure 2.1 shows two time series simulated from the AdINAR(1) model with mixture weight parameters $w=0.1$ and $w=0.9$, while keeping the other parameters fixed at $(\alpha, \theta, \lambda)=(0.10,0.15,5.66)$. The first series has mean count equal to 6 and variance 6.6 , while the second series, which gives more weight to the geometric component of the innovations, is overdispersed, with mean count equal to 6.2 and variance 33.

Formally, it is possible to analyze the role of the weight mixture $w$. Let $I(w)=\frac{\operatorname{Var}\left(Z_{t} \mid \theta, \lambda, w\right)}{E\left(Z_{t} \mid \theta, \lambda, w\right)}$ be the overdispersion index of the innovations. It is straightforward to show that the function $I(w)$ attains its maximum value at the point 1 if, and only if, $\mu_{g}^{2}-3 \mu_{g} \mu_{p}-\mu_{p}^{2}>0$, in which $\mu_{g}=\frac{1-\theta}{\theta}$ and $\mu_{p}=\lambda$ are mean of the Geometric and Poisson distributions, respectively. Therefore, under this condition, $I(w)$ is an increasing function for $w \in[0,1]$, and the level of overdispersion increases with the weight mixture $w$. As a consequence, the distribution of the innovations in the AdINAR(1) model accomodates the overdispersion in an adaptive manner.

We now derive the likelihood function of the $\operatorname{AdINAR}(1)$ model. Let $y=\left(y_{1}, \ldots, y_{T}\right)$ denote the values of an observed time series. For simplicity, we assume that $Y_{1}=y_{1}$ with probability one. Since the process $\left\{Y_{t}\right\}_{t \geq 1}$ is Markovian, the joint distribution of $Y_{1}, \ldots, Y_{T}$, given the parameters, can be factored as

$$
\operatorname{Pr}\left\{Y_{1}=y_{1}, \ldots, Y_{T}=y_{T} \mid \alpha, \theta, \lambda, w\right\}=\prod_{t=2}^{T} \operatorname{Pr}\left\{Y_{t}=y_{t} \mid Y_{t-1}=y_{t-1}, \alpha, \theta, \lambda, w\right\}
$$

Since, with probability one, $\alpha \circ Y_{t-1} \leq Y_{t-1}$ and $Z_{t} \geq 0$, by the law of total probability and the definition of the AdINAR(1) model we have that 
$w=0.1$

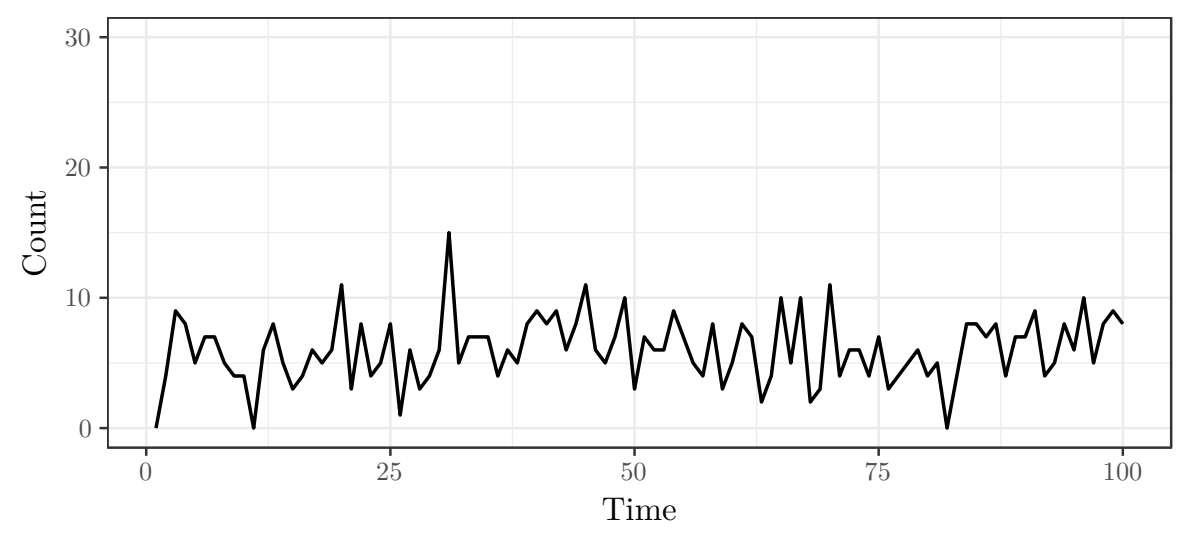

$w=0.9$

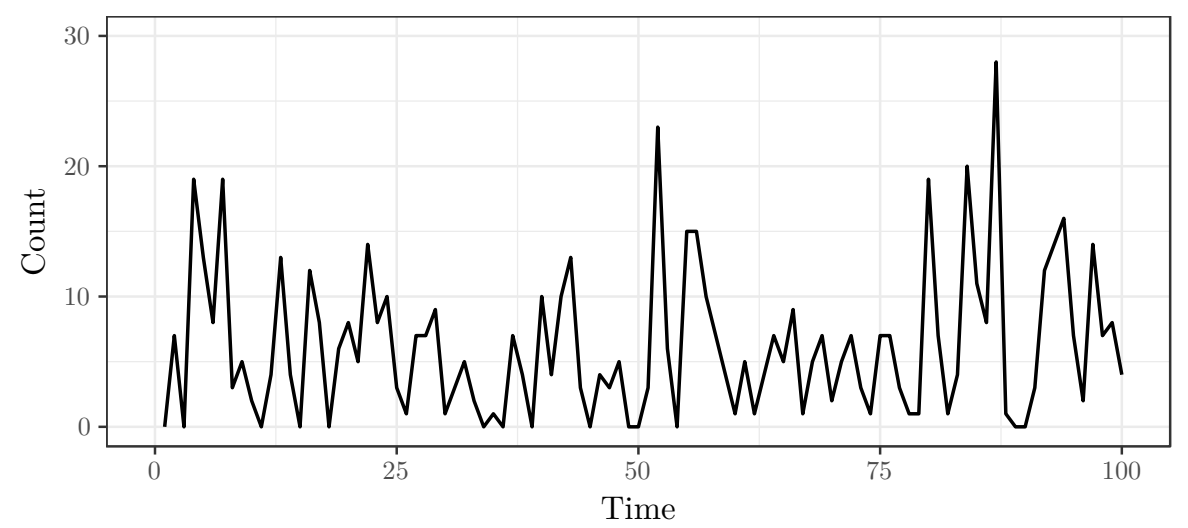

Figure 2.1: Simulated time series from the AdINAR(1) model, exhibiting the behavior of the model for two extreme values of the mixture weight parameter $w$.

$$
\begin{aligned}
\operatorname{Pr}\left\{Y_{t}=y_{t} \mid Y_{t-1}=y_{t-1}, \alpha, \theta, \lambda, w\right\}=\operatorname{Pr}\left\{\alpha \circ Y_{t-1}+Z_{t}=y_{t} \mid Y_{t-1}=y_{t-1}, \alpha, \theta, \lambda, w\right\} \\
=\operatorname{Pr}\left\{\sum_{i=1}^{Y_{t-1}} B_{i}(t)+Z_{t}=y_{t} \mid Y_{t-1}=y_{t-1}, \alpha, \theta, \lambda, w\right\} \\
=\sum_{m_{t}=0}^{\min \left\{y_{t}, y_{t-1}\right\}} \operatorname{Pr}\left\{\sum_{i=1}^{y_{t-1}} B_{i}(t)=m_{t}, Z_{t}=y_{t}-m_{t} \mid \alpha, \theta, \lambda, w\right\} \\
=\sum_{m_{t}=0}^{\min \left\{y_{t}, y_{t-1}\right\}} \operatorname{Pr}\left\{\sum_{i=1}^{y_{t-1}} B_{i}(t)=m_{t} \mid \alpha\right\} \operatorname{Pr}\left\{Z_{t}=y_{t}-m_{t} \mid \theta, \lambda, w\right\} .
\end{aligned}
$$

Hence, the AdINAR(1) model likelihood function is given by

$$
\begin{aligned}
& L_{y}(\alpha, \theta, \lambda, w)= \\
& \quad \prod_{t=2}^{T} \sum_{m_{t}=0}^{\min \left\{y_{t-1}, y_{t}\right\}}\left(\begin{array}{c}
y_{t-1} \\
m_{t}
\end{array}\right) \alpha^{m_{t}}(1-\alpha)^{y_{t-1}-m_{t}}\left(w \times \theta(1-\theta)^{y_{t}-m_{t}}+(1-w) \times \frac{e^{-\lambda} \lambda^{y_{t}-m_{t}}}{\left(y_{t}-m_{t}\right) !}\right) .
\end{aligned}
$$


In the next section, we show how the introduction of certain latent (unobservable) random variables allows us to specify the AdINAR(1) model in terms of a set of conditional distributions. This alternative representation leads to a factorization of the model's joint distribution which is of key importance to our Monte Carlo simulations.

\subsection{Data augmentation}

In the AdINAR(1) model, suppose that, in addition to the values of the counts $Y_{1}, \ldots, Y_{T}$, we could observe the values of the maturations $M_{t}=\alpha \circ Y_{t-1}$, as well as the values of a set of mixture component indicators $U_{t} \in\{0,1\}$, for $t=2, \ldots, T$. The $M_{t}$ 's would inform us the number of cases that matured from the previous epoch, breaking down $Y_{t}$ into two parcels (maturations plus innovations), while the $U_{t}$ 's would tell us from which component of the mixture the value of the $Z_{t}$ 's were "generated" in one realization of the process.

We postulate that

$$
\left(U_{t} \mid w\right) \sim \operatorname{Bernoulli}(w)
$$

and

$$
\left(M_{t} \mid \alpha, Y_{t-1}=y_{t-1}\right) \sim \operatorname{Binomial}\left(y_{t-1}, \alpha\right)
$$

Furthermore, we assume that

$$
\operatorname{Pr}\left\{Y_{t}=y_{t} \mid M_{t}=m_{t}, U_{t}=u_{t}, \theta, \lambda\right\}= \begin{cases}\theta(1-\theta)^{y_{t}-m_{t}} \mathbb{I}_{\left\{m_{t}, m_{t}+1, \ldots\right\}}\left(y_{t}\right) & \text { if } u_{t}=1 \\ \frac{e^{-\lambda} \lambda^{y_{t}-m_{t}}}{\left(y_{t}-m_{t}\right) !} \mathbb{I}_{\left\{m_{t}, m_{t}+1, \ldots\right\}}\left(y_{t}\right) & \text { if } u_{t}=0\end{cases}
$$

in which $\mathbb{I}_{A}$ denotes the indicator function of the set $A$, defined by $\mathbb{I}_{A}(x)=1$, if $x \in A$, and $\mathbb{I}_{A}(x)=0$, if $x \notin A$. 
Using the law of total probability and the product rule, we have that

$$
\begin{aligned}
& \operatorname{Pr}\left\{Y_{t}=y_{t} \mid Y_{t-1}=y_{t-1}, \alpha, \theta, \lambda, w\right\} \\
& =\sum_{m_{t}=0}^{y_{t-1}} \sum_{u_{t} \in\{0,1\}} \operatorname{Pr}\left\{Y_{t}=y_{t}, M_{t}=m_{t}, U_{t}=u_{t} \mid Y_{t-1}=y_{t-1}, \alpha, \theta, \lambda, w\right\} \\
& =\sum_{m_{t}=0}^{y_{t-1}} \sum_{u_{t} \in\{0,1\}}\left(\operatorname{Pr}\left\{M_{t}=m_{t} \mid Y_{t-1}=y_{t-1}, \alpha\right\}\right. \\
& \left.\times \operatorname{Pr}\left\{Y_{t}=y_{t} \mid M_{t}=m_{t}, U_{t}=u_{t}, \theta, \lambda\right\} \operatorname{Pr}\left\{U_{t}=u_{t} \mid w\right\}\right) .
\end{aligned}
$$

Since

$$
\begin{aligned}
\mathbb{I}_{\left\{m_{t}, m_{t+1}, \ldots\right\}}\left(y_{t}\right) \times \mathbb{I}_{\left\{0,1, \ldots, y_{t-1}\right\}}\left(m_{t}\right) & =\mathbb{I}_{\left\{0,1, \ldots, y_{t}\right\}}\left(m_{t}\right) \times \mathbb{I}_{\left\{0,1, \ldots, y_{t-1}\right\}}\left(m_{t}\right) \\
& =\mathbb{I}_{\left\{0,1, \ldots, \min \left\{y_{t}, y_{t-1}\right\}\right\}}\left(m_{t}\right),
\end{aligned}
$$

using the Markovian property $(*)$, we come to the conclusion that this data augmented model [Tanner and Wong, 1987, Van Dyk and Meng, 2001] induces the same AdINAR(1) model likelihood function discussed in the previous section. A graphical representation of the data augmented AdINAR(1) model is given in Figure 2.2. In the graph, absence of an arrow connecting two random objects means that they are conditionally independent given their parents. The rectangle (plate) denotes replication of the graph elements (see Jordan [2004]). The shaded nodes represent the observable quantities. For convenience, this plate notation is slightly abused for the extreme epoch $t=2$.

In the following section, we take advantage of this alternative representation of the AdINAR(1) model by the data augmentation scheme to derive simple closed forms for the model parameters and latent variables full conditional distributions, after the respective prior distributions have been specified.

\subsection{Full conditional distributions}

For convenience, we adopt a simplified notation in the following derivations, using the same letters $p$ and $\pi$ to denote different probability functions or densities, with distinctions made clear from context. 


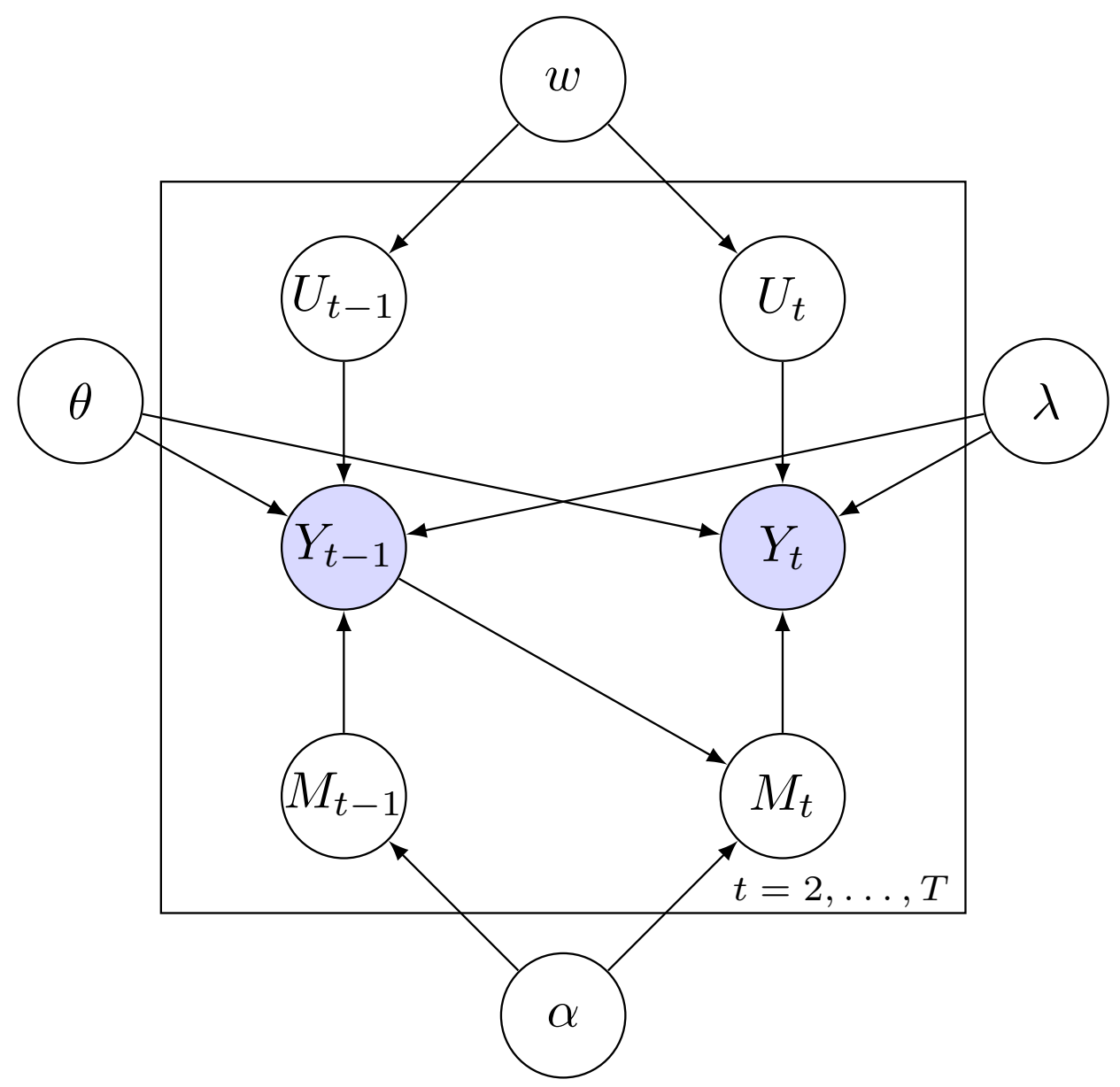

Figure 2.2: The data augmented AdINAR(1) model.

Let the prior distributions be

$$
\begin{array}{rlrl}
\alpha & \sim \operatorname{Beta}\left(a_{\alpha}, b_{\alpha}\right), & & \sim \operatorname{Gamma}\left(a_{\lambda}, b_{\lambda}\right), \\
\theta & \sim \operatorname{Beta}\left(a_{\theta}, b_{\theta}\right), & w \sim \operatorname{Beta}\left(a_{w}, b_{w}\right) .
\end{array}
$$

Define $m=\left(m_{2}, \ldots, m_{T}\right)$ and $u=\left(u_{2}, \ldots, u_{T}\right)$. From the graph in Figure 2.2, the joint distribution of the data augmented $\operatorname{AdINAR}(1)$ model factors as

$$
p(y, m, u, \alpha, \theta, \lambda, w)=\left(\prod_{t=2}^{T} p\left(y_{t} \mid m_{t}, u_{t}, \theta, \lambda\right) p\left(m_{t} \mid y_{t-1}, \alpha\right)\right) \pi(\alpha) \pi(\theta) \pi(\lambda) \pi(w)
$$

The following full conditional distributions are derived by inspection of this factorization.

$$
(\alpha \mid \text { all others }) \sim \operatorname{Beta}\left(a_{\alpha}+\sum_{t=2}^{T} m_{t}, b_{\alpha}+\sum_{t=2}^{T}\left(y_{t-1}-m_{t}\right)\right)
$$


$(\theta \mid$ all others $) \sim \operatorname{Beta}\left(a_{\theta}+\sum_{t=2}^{T} u_{t}, b_{\theta}+\sum_{t=2}^{T}\left(y_{t}-m_{t}\right) \mathbb{I}_{\{1\}}\left(u_{t}\right)\right)$

$(\lambda \mid$ all others $) \sim \operatorname{Gamma}\left(a_{\lambda}+\sum_{t=2}^{T}\left(y_{t}-m_{t}\right) \mathbb{I}_{\{0\}}\left(u_{t}\right), b_{\lambda}+(T-1)-\sum_{t=2}^{T} u_{t}\right)$
$(w \mid$ all others $) \sim \operatorname{Beta}\left(a_{w}+\sum_{t=2}^{T} u_{t}, b_{w}+(T-1)-\sum_{t=2}^{T} u_{t}\right)$

$\operatorname{Pr}\left\{U_{t}=1 \mid\right.$ all others $\} \propto w \theta(1-\theta)^{y_{t}-m_{t}}$

$\operatorname{Pr}\left\{U_{t}=0 \mid\right.$ all others $\} \propto(1-w) \frac{e^{-\lambda} \lambda^{y_{t}-m_{T}}}{\left(y_{t}-m_{t}\right) !}$,

for $t=2, \ldots, T$, in which $\propto$ denotes proportionality up to a proper normalization factor.

$$
\begin{aligned}
\operatorname{Pr}\left\{M_{t}\right. & \left.=m_{t} \mid \text { all others }\right\} \\
& \propto \begin{cases}\frac{1}{\left(y_{t-1}-m_{t}\right) ! m_{t} !}\left(\frac{\alpha}{(1-\theta)(1-\alpha)}\right)^{m_{t}} \mathbb{I}_{\left\{0,1, \ldots, \min \left\{y_{t}, y_{t-1}\right\}\right\}}\left(m_{t}\right) & \text { if } u_{t}=1 \\
\frac{1}{\left(y_{t}-m_{t}\right) !\left(y_{t-1}-m_{t}\right) ! m_{t} !}\left(\frac{\alpha}{\lambda(1-\alpha)}\right)^{m_{t}} \mathbb{I}_{\left\{0,1, \ldots, \min \left\{y_{t}, y_{t-1}\right\}\right\}}\left(m_{t}\right) & \text { if } u_{t}=0\end{cases}
\end{aligned}
$$

for $t=2, \ldots, T$.

Using these full conditional distributions, we code a Gibbs sampling [Gamerman and Lopes, 2006, Robert and Casella, 2013] scheme of the posterior distribution.

In the next section, we show how to use a sample from the posterior distribution to do fully probabilistic forecasts of future counts through the posterior predictive distribution.

\subsection{Bayesian forecasting}

Discarding the values of the latent variables, the Gibbs sampling scheme described in the previous section yields us, marginally, a sample $\left\{\alpha^{(n)}, \theta^{(n)}, \lambda^{(n)}, w^{(n)}\right\}_{n=1}^{N}$ from the posterior distribution of the model parameters. Uncertainty about future counts is represented by the 
$h$-steps-ahead posterior predictive distribution

$$
Y_{T+h} \mid Y_{1}=y_{1}, \ldots, Y_{T}=y_{T}
$$

for some target $h \geq 1$. In particular, a pointwise forecast is obtained as a suitable summary of this posterior predictive distribution.

To get a Monte Carlo approximation of the $h$-steps-ahead posterior predictive distribution, we use the AdINAR(1) model definition to propagate the process to the future sequentially, generating synthetic counts

$$
\begin{aligned}
y_{T+1}^{(n)} & =\alpha^{(n)} \circ y_{T}+z_{T+1}^{(n)}, \\
& \vdots \\
y_{T+h}^{(n)} & =\alpha^{(n)} \circ y_{T+h-1}^{(n)}+z_{T+h}^{(n)},
\end{aligned}
$$

for $n=1, \ldots, N$, in which the synthetic innovations $z_{T+1}^{(n)}, \ldots, z_{T+h}^{(n)}$ are drawn independently from a Geometric $\left(\theta^{(n)}\right)$ distribution, with probability $w^{(n)}$, or from a $\operatorname{Poisson}\left(\lambda^{(n)}\right)$ distribution, with probability $1-w^{(n)}$.

From the sample $\left\{y_{T+h}^{(n)}\right\}_{n=1}^{N}$, we approximate the $h$-steps-ahead posterior probability function by the respective empirical averages

$$
p\left(y_{T+h} \mid y_{1}, \ldots, y_{T}\right) \approx \frac{1}{N} \sum_{n=1}^{N} \mathbb{I}_{\left\{y_{T+h}\right\}}\left(y_{T+h}^{(n)}\right),
$$

for $y_{T+h} \geq 0$.

As a pointwise forecast $\hat{y}_{T+h}$, since we are dealing with discrete observations, we compute a generalized median of the $h$-steps-ahead posterior predictive distribution, defined by

$$
\hat{y}_{T+h}=\arg \min _{y_{T+h} \geq 0}\left|0.5-\sum_{r=0}^{y_{T+h}} p\left(r \mid y_{1}, \ldots, y_{T}\right)\right|
$$

\subsection{Simulation Study}

In this section, we carry out a simulation study to illustrate that the Gibbs Sampler algorithm described in Section 2.2 entails consistent estimates. We fix three configurations for the AdINAR(1) parameters in the simulation. The first scenario has parameters: 
$\left(\alpha_{0}, \theta_{0}, \lambda_{0}, w_{0}\right)=(0.10,0.15,5.66,0.1)$, while the second and third scenarios has the same parameters except that $w_{0}=0.50$ and $w_{0}=0.90$, respectively. We simulate time series with lengths $\{100,200,500,1000,5000\}$, and, for a specific configuration, we simulate 100 replications. In each replication, we calculate the marginal posterior means of the parameters and obtain the error of each estimate. For the hyperparameters, we make the choices $a_{\alpha}=1$, $b_{\alpha}=1, a_{\lambda}=1, b_{\lambda}=0.1, a_{\theta}=1, b_{\theta}=1, a_{w}=1$, and $b_{w}=1$, which correspond to reasonably flat priors. In all runs of the Gibbs samplers, we discard the first $10^{3}$ simulated values, which correspond to the burn-in period, and end up with a posterior sample of size $10^{4}$.

As a result, Tables 2.1, 2.2 and 2.3 shows the means of the Monte Carlo errors of each parameter, the difference between the posterior mean and the simulated value, of the AdINAR(1) model (standard deviations are given in parentheses). Table 2.2 shows, as expected, that the errors decrease as the time series length increases. Nevertheless, it is also important to inspect the extreme cases, $w_{0}=0.1$ and $w_{0}=0.9$, in Tables 2.1 and 2.3. Notice that the estimates of $\lambda$ are less accurate when $w$ tends to put more weight in the Geometric component, considering small and moderate sample sizes. Conversely, the estimates of $\theta$ tends to be less accurate when $w_{0}=0.1$, which favours the Poisson component in the mixture. Also, for time series whose length is greater than 500, the errors are substantially small. 


\begin{tabular}{c||ccccc}
\hline \hline$T$ & 100 & 200 & 500 & 1000 & 5000 \\
\hline \hline$\alpha$ & 0.0081 & 0.0031 & -0.0009 & 0.0029 & -0.0001 \\
$(\mathrm{sd})$ & $(0.0468)$ & $(0.0347)$ & $(0.0232)$ & $(0.0153)$ & $(0.0073)$ \\
$\theta$ & 0.0096 & -0.0003 & 0.0007 & 0.0004 & -0.0005 \\
$(\mathrm{sd})$ & $(0.0215)$ & $(0.0131)$ & $(0.0081)$ & $(0.0062)$ & $(0.0024)$ \\
$\lambda$ & 1.5777 & 0.6259 & 0.4299 & 0.3899 & -0.0155 \\
$(\mathrm{sd})$ & $(1.8416)$ & $(1.3578)$ & $(1.0203)$ & $(0.7629)$ & $(0.3732)$ \\
$w$ & -0.0581 & -0.0331 & -0.0096 & -0.0007 & 0.0006 \\
$(\mathrm{sd})$ & $(0.0734)$ & $(0.0464)$ & $(0.0460)$ & $(0.0377)$ & $(0.0191)$ \\
\hline \hline
\end{tabular}

Table 2.1: Means of the Monte Carlo errors for the 100 simulated time series from the AdINAR(1) with parameters for the $\left(\alpha_{0}, \theta_{0}, \lambda_{0}, w_{0}\right)=(0.10,0.15,5.66,0.9)$

\begin{tabular}{c||ccccc}
\hline \hline$T$ & 100 & 200 & 500 & 1000 & 5000 \\
\hline \hline$\alpha$ & -0.0162 & 0.0081 & 0.0036 & -0.0034 & -0.0001 \\
$(\mathrm{sd})$ & $(0.0334)$ & $(0.0513)$ & $(0.0346)$ & $(0.0243)$ & $(0.0092)$ \\
$\theta$ & -0.0008 & 0.0321 & 0.0014 & 0.0005 & -0.0001 \\
$(\mathrm{sd})$ & $(0.0227)$ & $(0.0417)$ & $(0.0094)$ & $(0.0062)$ & $(0.0031)$ \\
$\lambda$ & 0.3317 & 0.6722 & -0.0234 & 0.0469 & 0.0120 \\
$(\mathrm{sd})$ & $(0.72658)$ & $(0.2943)$ & $(0.2627)$ & $(0.2125)$ & $(0.0806)$ \\
$w$ & 0.0097 & 0.0286 & -0.0245 & -0.0069 & 0.0015 \\
$(\mathrm{sd})$ & $(0.0756)$ & $(0.0226)$ & $(0.0589)$ & $(0.0415)$ & $(0.0135)$ \\
\hline \hline
\end{tabular}

Table 2.2: Means of the Monte Carlo errors for the 100 simulated time series from the AdINAR(1) with parameters for the $\left(\alpha_{0}, \theta_{0}, \lambda_{0}, w_{0}\right)=(0.10,0.15,5.66,0.5)$

\begin{tabular}{c||ccccc}
\hline \hline$T$ & 100 & 200 & 500 & 1000 & 5000 \\
\hline \hline$\alpha$ & 0.0954 & -0.0124 & 0.0205 & -0.0019 & 0.0005 \\
$(\mathrm{sd})$ & $(0.0863)$ & $(0.0258)$ & $(0.0176)$ & $(0.0125)$ & $(0.0094)$ \\
$\theta$ & 0.2565 & 0.0629 & 0.0232 & 0.0103 & 0.0014 \\
$(\mathrm{sd})$ & $(0.1601)$ & $(0.1203)$ & $(0.0329)$ & $(0.0148)$ & $(0.0067)$ \\
$\lambda$ & -0.3559 & 0.1923 & -0.1335 & 0.0756 & -0.0338 \\
$(\mathrm{sd})$ & $(0.5818)$ & $(0.1856)$ & $(0.1081)$ & $(0.1217)$ & $(0.0678)$ \\
$w$ & 0.0168 & -0.0029 & -0.0072 & 0.007 & -0.0039 \\
$(\mathrm{sd})$ & $(0.0417)$ & $(0.0285)$ & $(0.0244)$ & $(0.0191)$ & $(0.0067)$ \\
\hline \hline
\end{tabular}

Table 2.3: Means of the Monte Carlo errors for the 100 simulated time series from the AdINAR(1) with parameters for the $\left(\alpha_{0}, \theta_{0}, \lambda_{0}, w_{0}\right)=(0.10,0.15,5.66,0.1)$ 


\section{Chapter 3}

\section{Semiparametric modeling of INAR}

\section{processes}

In this chapter, we develop a Bayesian hierarchical semiparametric model for phenomena related to time series of counts. The main feature of the model is its capability to learn a latent pattern of heterogeneity in the distribution of the process innovation rates, which are softly clustered through time with the help of a Dirichlet process placed at the top of the model hierarchy. In this sense, an infinite mixture model is induced on the innovation process, giving a more general structure when compared to the AdINAR model. Another property of this model is that the level of clustering is learned without specifying a priori the number of clusters of the mixture model. Furthermore, the probabilistic forecasting capabilities of the model are put to test in the analysis of crime data in Pittsburgh, with favorable results in comparison with the classical INAR(1) model.

\subsection{Generalized INAR(1) model}

We begin by generalizing the original INAR(1) model of McKenzie [1985] and Al-Osh and Alzaid [1988] as follows.

The generalized INAR(1) model is defined by the same functional relation of the AdI$\operatorname{NAR}(1)$, given in 2.1. The main difference is that the innovations $\left\{Z_{t}\right\}_{t \geq 2}$, given positive parameters $\left\{\lambda_{t}\right\}_{t \geq 2}$, be a sequence of conditionally independent Poisson $\left(\lambda_{t}\right)$ random variables. Notice that, in the homogeneous case, when all the $\lambda_{t}$ 's are assumed to be equal, we recover the original INAR $(1)$ model.

As in the $\operatorname{AdINAR}(1)$ developed in Chapter 2, this model can be interpreted as specifying a birth-and-death process, in which, at epoch $t$, the number of cases $Y_{t}$ is equal to the new 
cases $Z_{t}$ plus the cases that survived from the previous epoch; by contrast, under these assumptions, the innovations follow Poisson distributions with time-varying innovation rates.

Let $y=\left(y_{1}, \ldots, y_{T}\right)$ denote the values of an observed time series. For simplicity, we also assume here that $Y_{1}=y_{1}$ with probability one. Since the process $\left\{Y_{t}\right\}_{t \geq 1}$ is Markovian, the joint distribution of $Y_{1}, \ldots, Y_{T}$, given parameters $\alpha$ and $\lambda=\left(\lambda_{2}, \ldots, \lambda_{T}\right)$, can be factored as

$$
\operatorname{Pr}\left\{Y_{1}=y_{1}, \ldots, Y_{T}=y_{T} \mid \alpha, \lambda\right\}=\prod_{t=2}^{T} \operatorname{Pr}\left\{Y_{t}=y_{t} \mid Y_{t-1}=y_{t-1}, \alpha, \lambda_{t}\right\}
$$

Since, with probability one, $\alpha \circ Y_{t-1} \leq Y_{t-1}$ and $Z_{t} \geq 0$, by the law of total probability and the definition of the generalized $\operatorname{INAR}(1)$ model we have that

$$
\begin{aligned}
\operatorname{Pr}\left\{Y_{t}=y_{t} \mid Y_{t-1}=y_{t-1}, \alpha, \lambda_{t}\right\}=\operatorname{Pr}\left\{\alpha \circ Y_{t-1}+Z_{t}=y_{t} \mid Y_{t-1}=y_{t-1}, \alpha, \lambda_{t}\right\} \\
=\operatorname{Pr}\left\{\sum_{i=1}^{Y_{t-1}} B_{i}(t)+Z_{t}=y_{t} \mid Y_{t-1}=y_{t-1}, \alpha, \lambda_{t}\right\} \\
=\sum_{m_{t}=0}^{\min \left\{y_{t}, y_{t-1}\right\}} \operatorname{Pr}\left\{\sum_{i=1}^{y_{t-1}} B_{i}(t)=m_{t}, Z_{t}=y_{t}-m_{t} \mid \alpha, \lambda_{t}\right\} \\
=\sum_{m_{t}=0}^{\min \left\{y_{t}, y_{t-1}\right\}} \operatorname{Pr}\left\{\sum_{i=1}^{y_{t-1}} B_{i}(t)=m_{t} \mid \alpha\right\} \operatorname{Pr}\left\{Z_{t}=y_{t}-m_{t} \mid \lambda_{t}\right\} .
\end{aligned}
$$

Hence, the generalized INAR(1) model likelihood function is given by

$$
L_{y}(\alpha, \lambda)=\prod_{t=2}^{T} \sum_{m_{t}=0}^{\min \left\{y_{t-1}, y_{t}\right\}}\left(\begin{array}{c}
y_{t-1} \\
m_{t}
\end{array}\right) \alpha^{m_{t}}(1-\alpha)^{y_{t-1}-m_{t}}\left(\frac{e^{-\lambda_{t}} \lambda_{t}^{y_{t}-m_{t}}}{\left(y_{t}-m_{t}\right) !}\right) .
$$

In the next section, we show how the introduction of certain latent (unobservable) random variables allows us to specify the generalized INAR(1) model in terms of a set of conditional distributions. This alternative representation leads to a factorization of the model joint distribution which is the key element propelling our Monte Carlo simulations.

\subsection{Data augmentation}

In the generalized INAR(1) model, suppose that, in addition to the values of the counts $Y_{1}, \ldots, Y_{T}$, we could observe the values of the maturations $M_{t}=\alpha \circ Y_{t-1}$. The $M_{t}$ 's would tell us the number of cases that matured (survived) from the previous epoch, breaking down 
$Y_{t}$ into two parcels: maturations plus innovations.

This is another example of data augmentation [Tanner and Wong, 1987], in which the introduction of the unobservable maturations, with suitable conditional distributions, factors the model into more manageable pieces. Within this data augmentation scheme, we postulate that

$$
M_{t} \mid \alpha, Y_{t-1}=y_{t-1} \sim \operatorname{Binomial}\left(y_{t-1}, \alpha\right)
$$

and

$$
\operatorname{Pr}\left\{Y_{t}=y_{t} \mid M_{t}=m_{t}, \lambda_{t}\right\}=\frac{e^{-\lambda_{t}} \lambda_{t}^{y_{t}-m_{t}}}{\left(y_{t}-m_{t}\right) !} \mathbb{I}_{\left\{m_{t}, m_{t}+1, \ldots\right\}}\left(y_{t}\right)
$$

in which $\mathbb{I}_{A}$ denotes the indicator function of the set $A$, defined by $\mathbb{I}_{A}(x)=1$, if $x \in A$, and $\mathbb{I}_{A}(x)=0$, if $x \notin A$.

Using the law of total probability and the product rule, we have that

$$
\begin{aligned}
\operatorname{Pr}\left\{Y_{t}\right. & \left.=y_{t} \mid Y_{t-1}=y_{t-1}, \alpha, \lambda_{t}\right\}=\sum_{m_{t}=0}^{y_{t-1}} \operatorname{Pr}\left\{Y_{t}=y_{t}, M_{t}=m_{t} \mid Y_{t-1}=y_{t-1}, \alpha, \lambda_{t}\right\} \\
& =\sum_{m_{t}=0}^{y_{t-1}} \operatorname{Pr}\left\{Y_{t}=y_{t} \mid M_{t}=m_{t}, \lambda_{t}\right\} \times \operatorname{Pr}\left\{M_{t}=m_{t} \mid Y_{t-1}=y_{t-1}, \alpha\right\}
\end{aligned}
$$

in which, following the data augmentation scheme, we took advantage of the appropriate conditional independences.

Since

$$
\begin{aligned}
\mathbb{I}_{\left\{m_{t}, m_{t}+1, \ldots\right\}}\left(y_{t}\right) \times \mathbb{I}_{\left\{0,1, \ldots, y_{t-1}\right\}}\left(m_{t}\right) & =\mathbb{I}_{\left\{0,1, \ldots, y_{t}\right\}}\left(m_{t}\right) \times \mathbb{I}_{\left\{0,1, \ldots, y_{t-1}\right\}}\left(m_{t}\right) \\
& =\mathbb{I}_{\left\{0,1, \ldots, \min \left\{y_{t}, y_{t-1}\right\}\right\}}\left(m_{t}\right),
\end{aligned}
$$

comparing the expression above for $\operatorname{Pr}\left\{Y_{t}=y_{t} \mid Y_{t-1}=y_{t-1}, \alpha, \lambda_{t}\right\}$ with the results in the previous section, we come to the conclusion that this is a valid data augmentation scheme, since it induces the same generalized INAR(1) model likelihood function.

In the next section, we recollect the main definitions and results related to the Dirichlet Process which are necessary to build-up our semiparametric hierarchical model. The data augmentation scheme developed above will come in handy in the derivation of the full conditional distributions of the complete model. 


\subsection{The Dirichlet process}

Suppose that we represent our uncertainties about quantities assuming values in a sampling space $\mathscr{X}$, with sigma-field $\mathscr{B}$, by means of an underlying probability space $(\Omega, \mathscr{F}, \operatorname{Pr})$.

A mapping $\mathbb{G}: \mathscr{B} \times \Omega \rightarrow[0,1]$ is a random probability measure if $\mathbb{G}(\cdot, \omega)$ is a probability measure over $(\mathscr{X}, \mathscr{B})$, for every $\omega \in \Omega$, and $\mathbb{G}(B)=\mathbb{G}(B, \cdot)$ is a random variable, for each $B \in \mathscr{B}$.

Ferguson [1973] defined a random probability measure $\mathbb{G}$ descriptively as follows. Let $\beta$ be a finite nonzero measure over $(\mathscr{X}, \mathscr{B})$ and postulate that for each $\mathscr{B}$-measurable partition $\left\{B_{1}, \ldots, B_{k}\right\}$ of $\mathscr{X}$ the random vector $\left(\mathbb{G}\left(B_{1}\right), \ldots, \mathbb{G}\left(B_{k}\right)\right)$ has the ordinary Dirichlet distribution with parameters $\left(\beta\left(B_{1}\right), \ldots, \beta\left(B_{k}\right)\right)$. In this case, we say that $\mathbb{G}$ is a Dirichlet process with base measure $\beta$, and use the notation $\mathbb{G} \sim \mathrm{DP}(\beta)$. Ferguson proved that $\mathbb{G}$ is a properly defined random process in the sense of Kolmogorov's consistency theorem.

Defining the concentration parameter $\tau=\beta(\mathscr{X})$, and the base probability measure $G_{0}$ by $G_{0}(B)=\beta(B) / \beta(\mathscr{X})$, it follows from the usual properties of the Dirichlet distribution that $\mathrm{E}[\mathbb{G}(B)]=G_{0}(B)$ and $\operatorname{Var}[\mathbb{G}(B)]=G_{0}(B)\left(1-G_{0}(B)\right) /(\tau+1)$, for every $B \in \mathscr{B}$. Therefore, $\mathbb{G}$ is centered on $G_{0}$, and $\tau$ controls the concentration of $\mathbb{G}$ around $G_{0}$. In terms of the concentration parameter and the base probability measure, we write $\mathbb{G} \sim \operatorname{DP}\left(\tau G_{0}\right)$.

Inference with the Dirichlet process is tractable. In particular, Ferguson proved that the Dirichlet process is closed under sampling: if $X_{1}, \ldots, X_{n}$ are conditionally independent and identically distributed, given $\mathbb{G} \sim \operatorname{DP}\left(\tau G_{0}\right)$, such that $\operatorname{Pr}\left\{X_{i} \in B \mid \mathbb{G}=G\right\}=G(B)$, for every $B$ in $\mathscr{B}$, then

$$
\mathbb{G} \mid X_{1}=x_{1}, \ldots, X_{n}=x_{n} \sim \operatorname{DP}\left((\tau+n)\left(\frac{\tau}{\tau+n} G_{0}+\frac{1}{\tau+n} \sum_{i=1}^{n} \mathbb{I}_{B}\left(x_{i}\right)\right)\right)
$$

Notice that, using the law of total expectation, we have

$$
\begin{aligned}
\operatorname{Pr}\left\{X_{n+1} \in B \mid X_{1}, \ldots, X_{n}\right\} & =\mathrm{E}\left[\operatorname{Pr}\left\{X_{n+1} \in B \mid \mathbb{G}, X_{1}, \ldots, X_{n}\right\} \mid X_{1}, \ldots, X_{n}\right] \\
& =\mathrm{E}\left[\operatorname{Pr}\left\{X_{n+1} \in B \mid \mathbb{G}\right\} \mid X_{1}, \ldots, X_{n}\right] \\
& =\mathrm{E}\left[\mathbb{G}(B) \mid X_{1}, \ldots, X_{n}\right],
\end{aligned}
$$

almost surely, for every $B$ in $\mathscr{B}$, in which the second equality follows from the conditional 
independence of the $X_{i}$ 's. Hence, the posterior predictive distribution is

$$
\operatorname{Pr}\left\{X_{n+1} \in B \mid X_{1}=x_{1}, \ldots, X_{n}=x_{n}\right\}=\frac{\tau}{\tau+n} G_{0}(B)+\frac{1}{\tau+n} \sum_{i=1}^{n} I_{B}\left(x_{i}\right) .
$$

This expression of the posterior predictive distribution unleashes important features of the Dirichlet process, thereby showing how it can be used as a modeling tool. In particular, it defines a data generating process known as the Pólya-Blackwell-MacQueen urn [Blackwell and MacQueen, 1973]. If we imagine the sequential generation of the $X_{i}$ 's, for $i=1, \ldots, n$, we see that a value is generated anew from $G_{0}$ with probability proportional to $\tau$, or we repeat one the previously generated values with probability proportional to its multiplicity. This shows that, almost surely, realizations of a Dirichlet process $\mathbb{G}$ are discrete probability measures, maybe with denumerably infinite support, depending on the nature of $G_{0}$. Also, this data generating process associated with the Pólya-Blackwell-MacQueen urn implies that the $X_{i}$ 's are clustered, which is the main feature of the Dirichlet process that we rely on to build our semiparametric model. Antoniak [1974] derived the conditional distribution of the number of distinct $X_{i}^{\prime}$ 's, that is, the number of clusters $K$, given the concentration parameter $\tau$, as

$$
\operatorname{Pr}\{K=k \mid \tau\}=S(n, k) \tau^{k} \frac{\Gamma(\tau)}{\Gamma(\tau+n)} \mathbb{I}_{\{1,2, \ldots, n\}}(k),
$$

in which $S(n, k)$ denotes the unsigned Stirling number of the first kind.

In the next section, we place a Dirichlet process at the top of the hierarchy of the generalized INAR(1) model, completing the specification of our semiparametric model, thereby being able to represent our uncertainty about the values of the unobservable innovation rates $\lambda_{t}$ 's, given the information contained in the observed counts. In doing so, we benefit from the clustering properties of the Dirichlet process described above, identifying different regimes for the innovation rates as the process evolves through time.

\subsection{DP-INAR(1) model}

The DP-INAR(1) model completes the generalized INAR(1) model defined in Section 3.1, placing a Dirichlet process at the top of the hierarchy. Formally, we model the innovation rates $\lambda_{2}, \ldots, \lambda_{T}$, given $\mathbb{G} \sim \operatorname{DP}\left(\tau G_{0}\right)$, as conditionally independent and identically distributed, with $\operatorname{Pr}\left\{\lambda_{t} \in B \mid \mathbb{G}=G\right\}=G(B)$, for every Borel set $B$. The prior distributions for $\alpha$ and $\tau$ are $\operatorname{Beta}\left(a_{\alpha}, b_{\alpha}\right)$ and $\operatorname{Gamma}\left(a_{\tau}, b_{\tau}\right)$, respectively. The base probability measure 
$G_{0}$ is a Gamma $\left(a_{0}, b_{0}\right)$ distribution. In Section 3.5, we discuss in detail the choice of prior parameters. In summary, the hierarchical structure of the DP-INAR(1) is given by:

$$
\left\{\begin{array}{l}
Y_{t}=\alpha \circ Y_{t-1}+Z_{t} \\
\alpha \sim \operatorname{Beta}\left(a_{\alpha}, b_{\alpha}\right) \\
Z_{t} \mid \lambda_{t} \sim \operatorname{Poisson}\left(\lambda_{t}\right) \\
\lambda_{t} \mid \mathbb{G} \sim \mathbf{G} \\
\mathbb{G} \sim \operatorname{DP}\left(\tau G_{0}\right) \\
G_{0} \sim \operatorname{Gama}\left(a_{0}, b_{0}\right) \\
\tau \sim \operatorname{Gamma}\left(a_{\tau}, b_{\tau}\right),
\end{array}\right.
$$

for $t=2, \ldots, T$. Figure 3.1 displays a graphical representation of the DP-INAR(1) model (see Jordan [2004] for a witful discussion of graphical models).

Our next step is to derive the full conditional distributions for all latent variables and model parameters. For convenience, we adopt a simplified notation in the following derivations, using the same letter $p$ to denote different probability functions or densities, with distinctions made clear from the context.

Define $m=\left(m_{2}, \ldots, m_{T}\right)$, and let $\mu_{\mathbb{G}}$ denote the distribution of $\mathbb{G}$. Marginalizing $\mathbb{G}$ on the graph, we have

$$
\begin{aligned}
p(y, m, \alpha, \lambda) & =\int p(y, m, \alpha, \lambda \mid G) d \mu_{\mathbb{G}}(G) \\
& =\left\{\prod_{t=2}^{T} p\left(y_{t} \mid m_{t}, \lambda_{t}\right) p\left(m_{t} \mid y_{t-1}, \alpha\right)\right\} \times \pi(\alpha) \times \int \prod_{t=2}^{T} p\left(\lambda_{t} \mid G\right) d \mu_{\mathbb{G}}(G) .
\end{aligned}
$$

In this expression, the last integral is the joint distribution $p\left(\lambda_{2}, \ldots, \lambda_{T}\right)$, pointing out that the random vector $\left(\lambda_{2}, \ldots, \lambda_{T}\right)$ has an exchangeable distribution. Due to this distributional symmetry and the product rule, we can always make $p\left(\lambda_{2}, \ldots, \lambda_{T}\right)$ depend on a certain $\lambda_{t}$ only through $p\left(\lambda_{t} \mid \lambda_{\backslash t}\right)$, in which $\lambda_{\backslash t}$ denotes the vector $\lambda$ with the component $\lambda_{t}$ removed. Using the symbol $\propto$ to denote proportionality up to a suitable normalization factor, and the label "all others" to designate the observed counts $y$, and all the other latent variables and model parameters, with the exception of the one under consideration, we have 


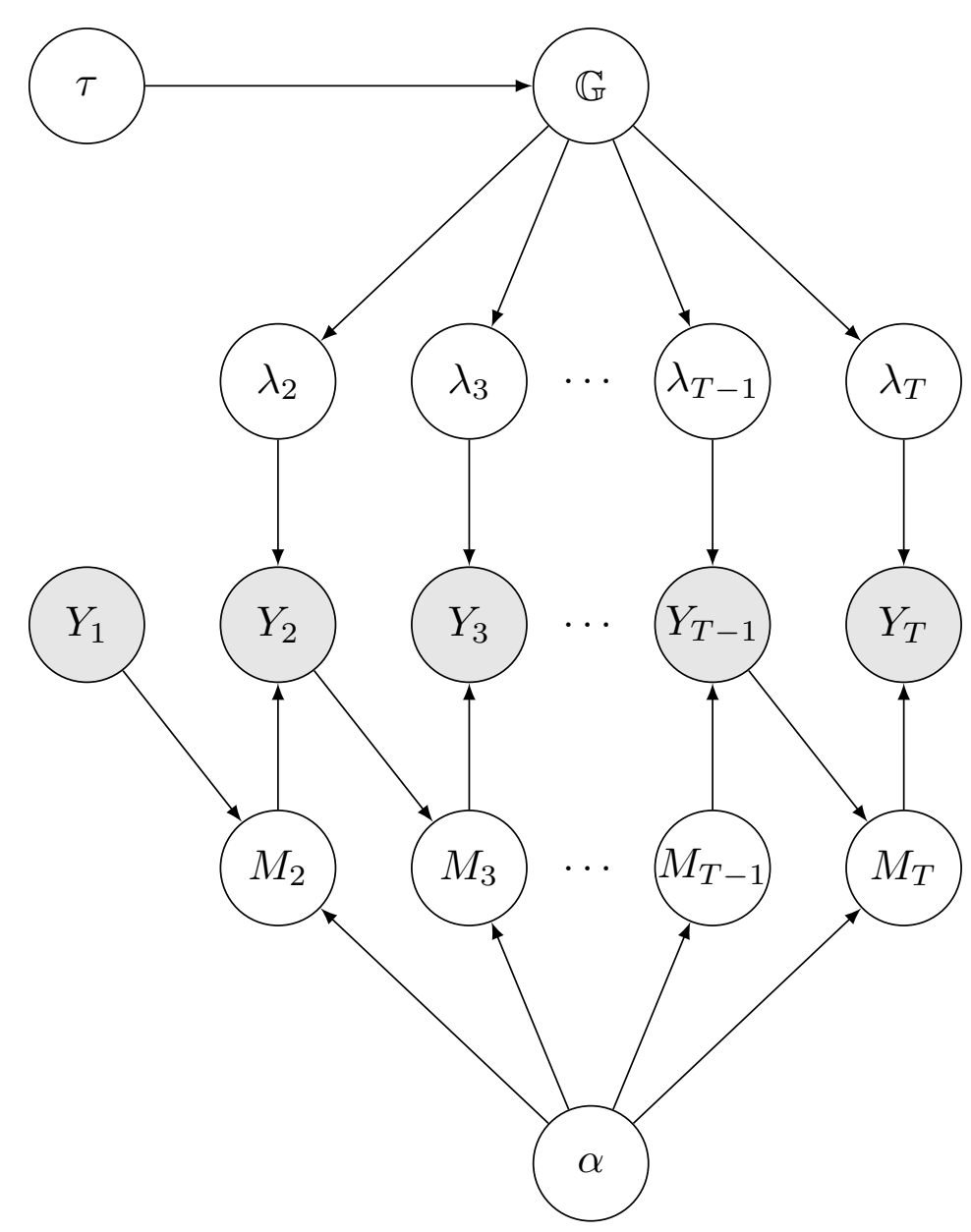

Figure 3.1: The data augmented DP-INAR(1) model.

that

$p\left(\lambda_{t} \mid\right.$ all others $) \propto p(y, m, \alpha, \lambda) \propto p\left(\lambda_{t} \mid \lambda_{\backslash t}\right) p\left(y_{t} \mid m_{t}, \lambda_{t}\right) \propto e^{-\lambda_{t}} \lambda_{t}^{y_{t}-m_{t}} p\left(\lambda_{t} \mid \lambda_{\backslash t}\right)$.

Therefore, the Pólya-Blackwell-MacQueen urn process yields the full conditional distribution of $\lambda_{t}$ as the mixture

$$
\begin{aligned}
\lambda_{t} \mid \text { all others } \sim & \frac{\tau \cdot b_{0}^{a_{0}} \cdot \Gamma\left(y_{t}-m_{t}+a_{0}\right)}{\Gamma\left(a_{0}\right)\left(b_{0}+1\right)^{y_{t}-m_{t}+a_{0}}} \times \operatorname{Gamma}\left(y_{t}-m_{t}+a_{0}, b_{0}+1\right) \\
& +\sum_{r \neq t} \lambda_{r}^{y_{t}-m_{t}} e^{-\lambda_{r}} \delta_{\left\{\lambda_{r}\right\}},
\end{aligned}
$$

in which $\delta_{\left\{\lambda_{r}\right\}}$ denotes a point mass at $\lambda_{r}$. In the former expression we suppressed the normalization constant which makes all mixture weights add up to one. 
The derivations of the full conditionals for $\alpha$ and $m_{t}$ are straightforward.

$$
\alpha \mid \text { all others } \sim \operatorname{Beta}\left(a_{\alpha}+\sum_{t=2}^{T} m_{t}, b_{\alpha}+\sum_{t=2}^{T}\left(y_{t-1}-m_{t}\right)\right) .
$$

$$
p\left(m_{t} \mid \text { all others }\right) \propto \frac{1}{m_{t} !\left(y_{t}-m_{t}\right) !\left(y_{t-1}-m_{t}\right) !}\left(\frac{\alpha}{\lambda_{t}(1-\alpha)}\right)^{m_{t}} \mathbb{I}_{\left\{0,1, \ldots, \min \left\{y_{t-1}, y_{t}\right\}\right\}}\left(m_{t}\right) .
$$

West [1992] shows how to derive the full conditional distribution of the concentration parameter $\tau$ in simple closed form, after the introduction of an auxiliary random variable $U$ which allows us to build a Gibbs sampler for $\tau$. Using this technique, we have the full conditionals

$$
U \text { all others } \sim \operatorname{Beta}(\tau+1, T-1)
$$

$$
\begin{aligned}
\tau \mid \text { all others } \sim & \frac{\Gamma\left(a_{\tau}+k\right)}{\left(b_{\tau}-\log u\right)^{a_{\tau}+k-1}} \times \operatorname{Gamma}\left(a_{\tau}+k, b_{\tau}-\log u\right) \\
& \quad+\frac{(T-1) \cdot \Gamma\left(a_{\tau}+k-1\right)}{\left(b_{\tau}-\log u\right)^{a_{\tau}+k-1}} \times \operatorname{Gamma}\left(a_{\tau}+k-1, b_{0}^{(\tau)}-\log u\right),
\end{aligned}
$$

in which we suppressed the normalization constant which makes the two mixture weights add up to one.

These full conditional distributions allow us to explore the model posterior distribution by coding a plain Gibbs sampler [Gamerman and Lopes, 2006]. Experimentation with this Gibbs sampler shows that, as pointed out by Escobar and West [Escobar and West, 1998] in a similar context, we can improve mixing by resampling simultaneously the values of all $\lambda_{t}$ 's inside the same cluster at the end of each iteration. Formally, let $\left(\lambda_{1}^{*}, \ldots, \lambda_{k}^{*}\right)$ be the $k$ unique values among $\left(\lambda_{2}, \ldots, \lambda_{T}\right)$ and define the number of occupants of cluster $j$ by $n_{j}=\sum_{t=2}^{T} \mathbb{I}_{\left\{\lambda_{j}^{*}\right\}}\left(\lambda_{t}\right)$. It follows that

$$
f\left(\lambda_{j}^{*} \mid \text { all others }\right) \propto \prod_{\left\{t: \lambda_{t}=\lambda_{j}^{*}\right\}} p\left(y_{t} \mid m_{t}, \lambda_{t}\right) g_{0}\left(\lambda_{t}\right) \propto\left(\lambda_{j}^{*}\right)^{a_{0}+\sum_{t=2}^{T}\left(y_{t}-m_{t}\right)-1 \cdot \mathbb{I}_{\lambda_{j}^{*}}\left(\lambda_{t}\right)} e^{-\lambda_{j}^{*}\left(b_{0}+n_{j}\right)}
$$

which entails that 


$$
\lambda_{j}^{*} \mid \text { all others } \sim \operatorname{Gamma}\left(a_{0}+\sum_{t=2}^{T}\left(y_{t}-m_{t}\right) \cdot \mathbb{I}_{\left\{\lambda_{j}^{*}\right\}}\left(\lambda_{t}\right), b_{0}+n_{j}\right) .
$$

for $j=1, \ldots, k$. After the $\lambda_{j}^{*}$ s are sampled from this distribution, we update the values of all $\lambda_{t}$ 's inside each cluster by the corresponding $\lambda_{j}^{*}$.

In the next section, we discuss how to choose the prior parameters for the $\operatorname{DP}-\operatorname{INAR}(1)$ model.

\subsection{Choice of prior parameters}

Extending the original scheme proposed by Dorazio [2009], we choose the parameters $a_{\tau}$ and $b_{\tau}$ of the $\tau$ prior by minimizing the Kullback-Leibler divergence between the prior distribution of the number of clusters $K$ and a uniform discrete distribution on a suitable range. Using the results in Section 3.3, the marginal probability function of $K$ can be computed as

$$
\pi(k)=\int_{0}^{\infty} \operatorname{Pr}\{K=k \mid \tau\} \pi(\tau) d \tau=\frac{b_{\tau} S(T-1, k)}{\Gamma\left(a_{\tau}\right)} I\left(a_{\tau}, b_{\tau} ; k\right),
$$

for $k=1, \ldots, T-1$, in which

$$
I\left(a_{\tau}, b_{\tau} ; k\right)=\int_{0}^{\infty} \frac{\tau^{k+a_{\tau}-1} e^{-b_{\tau} \tau} \Gamma(\tau)}{\Gamma(\tau+T-1)} d \tau
$$

Using the information available about the phenomena under consideration to make a sensible choice for the integers $k_{\min }$ and $k_{\max }$, and letting $q$ be the probability function of a uniform discrete distribution on $\left\{k_{\min }, \ldots, k_{\max }\right\}$, that is

$$
q(k)=\frac{1}{\left(k_{\max }-k_{\min }+1\right)} \mathbb{I}_{\left\{k_{\min }, \ldots, k_{\max }\right\}}(k),
$$

we find, by numerical integration and optimization, the values of $a_{\tau}$ and $b_{\tau}$ that minimize the Kullback-Leibler divergence

$$
\begin{aligned}
\mathrm{KL}[\pi & \| q]=\sum_{k=k_{\min }}^{k_{\max }} q(k) \log \left(\frac{q(k)}{\pi(k)}\right) \\
& =(\text { constant })+\log \Gamma\left(a_{\tau}\right)-a_{\tau} \log b_{\tau}-\frac{1}{\left(k_{\max }-k_{\min }+1\right)} \sum_{k=k_{\min }}^{k_{\max }} \log I\left(a_{\tau}, b_{\tau} ; k\right) .
\end{aligned}
$$

We choose the parameters $a_{0}$ and $b_{0}$ of the base probability density $g_{0}$ in a similar 
fashion, minimizing the Kullback-Leibler divergence between $g_{0}$ and a uniform distribution on a suitable range $\left[0, \lambda_{\max }\right]$, in which $\lambda_{\max }$ is chosen by taking into consideration the available information on the studied phenomena. Letting $h$ be a uniform density on $\left[0, \lambda_{\max }\right]$, that is

$$
h(\lambda)=\left(\frac{1}{\lambda_{\max }}\right) \mathbb{I}_{\left[0, \lambda_{\max }\right]}(\lambda)
$$

we find, by numerical optimization, the values of $a_{0}$ and $b_{0}$ that minimize the KullbackLeibler divergence

$$
\begin{aligned}
\mathrm{KL} & {\left[g_{0} \| h\right]=\int_{0}^{\lambda_{\max }}\left(\frac{1}{\lambda_{\max }}\right) \log \left(\frac{1 / \lambda_{\max }}{g_{0}(\lambda)}\right) d \lambda } \\
& =-\log \lambda_{\max }-a_{0} \log b_{0}+\log \Gamma\left(a_{0}\right)-\left(a_{0}-1\right)\left(\log \lambda_{\max }-1\right)+\frac{b_{0} \lambda_{\max }}{2}
\end{aligned}
$$

Choosing the parameters for the $\alpha$ prior is more straightforward, with $a_{\alpha}=b_{\alpha}=1$ being a natural choice.

\subsection{Bayesian forecasting}

The Gibbs sampler described in Section 3.4 yields, marginally, a sample $\left\{\alpha^{(n)}, \lambda^{(n)}\right\}_{n=1}^{N}$ from the posterior distribution. Note that, for $n=1, \ldots, N$, we can obtain the number of clusters $k^{(n)}$ as the number of distinct entries in the vector $\lambda^{(n)}=\left(\lambda_{1}^{(n)}, \ldots, \lambda_{T}^{(n)}\right)$. Uncertainty about future counts is represented by the $h$-steps-ahead posterior predictive distribution

$$
Y_{T+h} \mid Y_{1}=y_{1}, \ldots, Y_{T}=y_{T}
$$

for some target $h \geq 1$. In particular, a pointwise forecast is obtained as a suitable summary of this posterior predictive distribution.

Using the law of total probability, the product rule, and simplifying the conditional independences in the model, we can write the posterior predictive probability function as

$$
\begin{aligned}
p\left(y_{T+h} \mid y_{1}, \ldots, y_{T}\right)=\int & p\left(y_{T+h} \mid y_{T}, \alpha, \lambda_{T+1}, \ldots, \lambda_{T+h}\right) \\
& \times \prod_{i=1}^{h} p\left(\lambda_{T+i} \mid \lambda_{2}, \ldots, \lambda_{T+i-1}\right) \\
& \times p\left(\alpha, \lambda_{2}, \ldots, \lambda_{T} \mid y_{1}, \ldots, y_{T}\right) d \alpha d \lambda_{2} \ldots d \lambda_{T+h} .
\end{aligned}
$$

A nice property of the DP-INAR(1) model is that we can derive a simple analytical 
expression for the first factor in the integrand above.

Proposition 3.6.1. The probability function of $Y_{t+h}$, given $Y_{t}=y_{t}, \alpha$, and $\left(\lambda_{t+1}, \ldots, \lambda_{t+h}\right)$, can be writen as the convolution of a $\operatorname{Bin}\left(y_{t}, \alpha^{h}\right)$ distribution and a Poisson $\left(\mu_{h}\right)$ distribution,

$$
p\left(y_{t+h} \mid y_{t}, \alpha, \lambda_{t+1}, \ldots, \lambda_{t+h}\right)=\sum_{m=0}^{\min \left\{y_{t}, y_{t+h}\right\}}\left(\begin{array}{c}
y_{t} \\
m
\end{array}\right)\left(\alpha^{h}\right)^{m}\left(1-\alpha^{h}\right)^{y_{t}-m}\left(\frac{\mu_{h}^{y_{t+h}-m} e^{-\mu_{h}}}{\left(y_{t+h}-m\right) !}\right)
$$

in which

$$
\mu_{h}=\sum_{i=1}^{h} \alpha^{h-i} \lambda_{t+i}
$$

Proof. We prove the result by induction. For $h=1$, using a simplified notation, the conditional moment generating function is given by

$$
M_{Y_{t+1} \mid Y_{t}}(s)=\mathrm{E}\left[e^{s Y_{t+1}} \mid Y_{t}\right]=\mathrm{E}\left[e^{s\left(\alpha \circ Y_{t}+Z_{t+1}\right)} \mid Y_{t}\right]=\mathrm{E}\left[e^{s\left(\sum_{i=1}^{Y_{t}} B_{i}(t)+Z_{t+1}\right)} \mid Y_{t}\right]
$$

But since $\left\{Z_{t}\right\}_{t \geq 2}$ is a sequence of conditionally independent random variables, which is also conditionally independent of $\left\{B_{i}(t): i \geq 0, t \geq 2\right\}$, we have that

$$
M_{Y_{t+1} \mid Y_{t}}(s)=\mathrm{E}\left[e^{s \sum_{i=1}^{Y_{t}} B_{i}(t)} \mid Y_{t}\right] \mathrm{E}\left[e^{s Z_{t+1}}\right]=\left(\alpha e^{s}+(1-\alpha)\right)^{Y_{t}} \exp \left(\lambda_{t+1}\left(e^{s}-1\right)\right),
$$

which is the product of the generating functions of a $\operatorname{Binomial}\left(Y_{t}, \alpha\right)$ random variable and a $\operatorname{Poisson}\left(\lambda_{t+1}\right)$ random variable. Now, suppose the result holds for an arbitrary $h \geq 2$. Then,

$$
\begin{aligned}
M_{Y_{t+h+1} \mid Y_{t}}(s) & =\mathrm{E}\left[e^{s Y_{t+h+1}} \mid Y_{t}\right]=\mathrm{E}\left[\mathrm{E}\left[e^{s Y_{t+h+1}} \mid Y_{t+h}\right] \mid Y_{t}\right] \\
& =\mathrm{E}\left[e^{u Y_{t+h}} \mid Y_{t}\right] \exp \left(\lambda_{t+h+1}\left(e^{s}-1\right)\right)
\end{aligned}
$$

in which we defined $e^{u}=\alpha e^{s}+(1-\alpha)$. Consequently, from the induction hypothesis, we have that

$$
\begin{aligned}
M_{Y_{t+h+1} \mid Y_{t}}(s)= & \left(\alpha^{h} e^{u}+\left(1-\alpha^{h}\right)\right)^{Y_{t}} \exp \left(\mu_{h}\left(e^{u}-1\right)\right) \exp \left(\lambda_{t+h+1}\left(e^{s}-1\right)\right) \\
= & \left(\alpha^{h}\left(\alpha e^{s}+(1-\alpha)\right)+\left(1-\alpha^{h}\right)\right)^{Y_{t}} \exp \left(\mu_{h}\left(\left(\alpha e^{s}+(1-\alpha)\right)-1\right)\right) \\
& \times \exp \left(\lambda_{t+h+1}\left(e^{s}-1\right)\right) \\
= & \left(\alpha^{h+1} e^{s}+\left(1-\alpha^{h+1}\right)\right)^{Y_{t}} \exp \left(\mu_{h+1}\left(e^{s}-1\right)\right),
\end{aligned}
$$

in which $\mu_{h+1}=\alpha \mu_{h}+\lambda_{t+h+1}$. Hence, the result holds for $h+1$, completing the proof. 


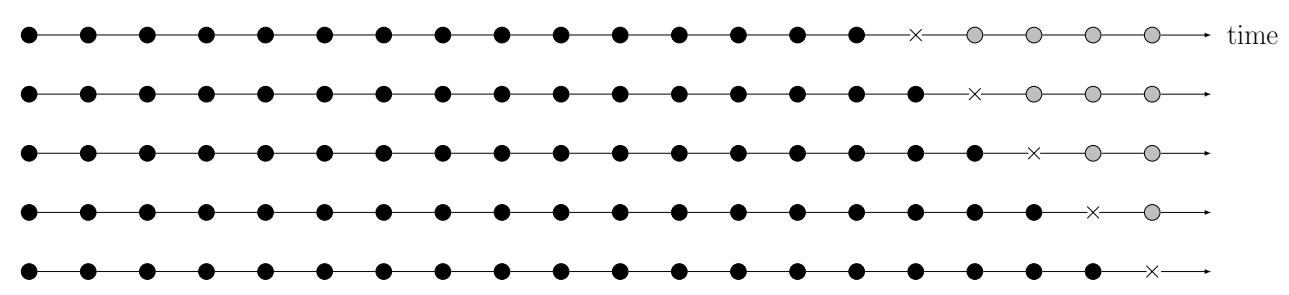

Figure 3.2: Cross-validation scheme for one-step-ahead predictions. For each line, the black dots indicate the training set. Predictions are made for the target epoch marked with an $\times$.

Using the Pólya-Blackwell-MacQueen urn process repeatedly, for $n=1 \ldots, N$, we draw a sample $\left\{\lambda_{T+1}^{(n)}, \ldots, \lambda_{T+h}^{(n)}\right\}_{n=1}^{N}$ from $\prod_{i=1}^{h} p\left(\lambda_{T+i} \mid \lambda_{2}, \ldots, \lambda_{T+i-1}\right)$ sequentially as follows:

$$
\begin{aligned}
\lambda_{T+1}^{(n)} & \sim \frac{\tau}{\tau+T} G_{0}+\frac{1}{\tau+T} \sum_{t=2}^{T} \delta_{\left\{\lambda_{t}^{(n)}\right\}} ; \\
\lambda_{T+2}^{(n)} & \sim \frac{\tau}{\tau+T+1} G_{0}+\frac{1}{\tau+T+1} \sum_{t=2}^{T+1} \delta_{\left\{\lambda_{t}^{(n)}\right\}} ; \\
& \vdots \\
\lambda_{T+h}^{(n)} & \sim \frac{\tau}{\tau+T+h-1} G_{0}+\frac{1}{\tau+T+h-1} \sum_{t=2}^{T+h-1} \delta_{\left\{\lambda_{t}^{(n)}\right\}} .
\end{aligned}
$$

Combining all these elements, we approximate the integral representation of the $h$-stepsahead posterior predictive probability function by the Monte Carlo average

$$
p\left(y_{T+h} \mid y_{1}, \ldots, y_{T}\right) \approx \frac{1}{N} \sum_{n=1}^{N} p\left(y_{T+h} \mid y_{T}, \alpha^{(n)}, \lambda_{T+1}^{(n)}, \ldots, \lambda_{T+h}^{(n)}\right)
$$

for $y_{T+h} \geq 0$.

As a pointwise forecast $\hat{y}_{T+h}$, we compute the generalized median of the $h$-steps-ahead posterior predictive distribution as in the $\operatorname{AdINAR}(1)$ model, that is,

$$
\hat{y}_{T+h}=\arg \min _{y_{T+h} \geq 0}\left|0.5-\sum_{r=0}^{y_{T+h}} p\left(r \mid y_{1}, \ldots, y_{T}\right)\right|
$$

We use a form of cross-validation to evaluate the forecasting performance of the model. For an observed time series $y_{1}, \ldots, y_{T}$, we pick some $T^{*}<T$, and treat the counts $y_{T^{*}}, \ldots, y_{T}$ as a holdout (test) sample. For $t \geq T^{*}$, we train the model conditioning only on the values $y_{1}, \ldots, y_{t-1}$ and making an $h$-steps-ahead prediction $\hat{y}_{t+h}$. To score the forecast performance, we average the median deviations $\left|\hat{y}_{t+h}-y_{t+h}\right|$ over all predictions. This cross-validation procedure is depicted in Figure 3.2. 
In the next section, we assess the forecasting performance of the DP-INAR(1) and AdINAR(1) models, analyzing monthly time series of burglary occurrences in Pittsburgh, USA.

\subsection{Burglary data}

In this section, we analyze monthly time series of burglary events in Pittsburgh, USA, from January 1990 to December 2001. The whole dataset can be found at: https://github.com/heltongraziadei/BayesINAR/blob/master/data/Pittsburgh.rda. In this dataset, each time series has a length of 144 months and corresponds to a certain patrol area, comprising a total of 36 time series.

Figure 3.3 presents the time series for patrol area 58, which displays substantial time heterogeneity and variation in the monthly counts of burglary events. In what follows, we use patrol area 58 to exemplify the training procedures for both the $\operatorname{AdINAR(1)}$ and the DP-INAR(1) models. This patrol area presents substantial overdispersion in the monthly counts of burglary events, with mean 10.4 and variance 31.1 .

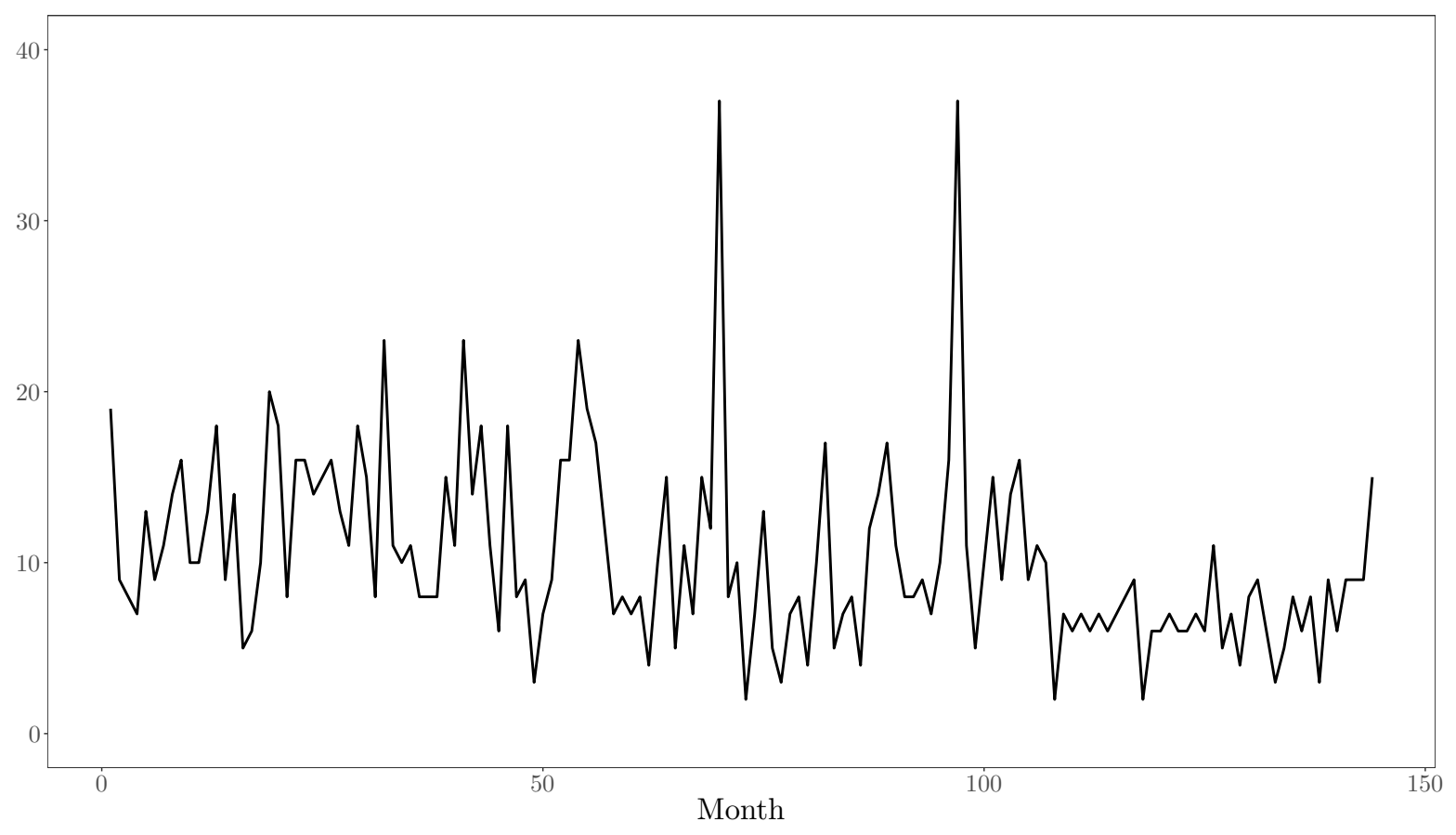

Figure 3.3: Monthly burglary events for patrol area 58.

In all runs of the Gibbs samplers, we discard the first $10^{3}$ simulated values, which correspond to the burn-in period, and end up with a posterior sample of size $10^{4}$.

For the AdINAR(1) model hyperparameters, we make the choices $a_{\alpha}=1, b_{\alpha}=1, a_{\lambda}=1$, $b_{\lambda}=0.1, a_{\theta}=1, b_{\theta}=1, a_{w}=1$, and $b_{w}=1$, which correspond to reasonably flat priors. 
Figure 3.4 displays the marginal posterior distributions of the AdINAR(1) model parameters. The posterior distribution of the thinning parameter $\alpha$ is fairly concentrated, with posterior mean 0.31, showing that the autoregressive component is not negligible for this patrol area. The posterior mean of $\lambda$ is 6.78 , while the posterior mean of $\theta$ is 0.12 . Also, the posterior distribution of $w$, with posterior mean 0.38 , shows that the geometric component of the mixture has less weight for this patrol area.

For the DP-INAR(1) model, we specify the hyperparameters as follows. To determine $a_{\tau}$ and $b_{\tau}$, the optimization procedure described in Section 3.5, with $k_{\min }=1$ and $k_{\max }=143$, yields $a_{\tau}=0.519$ and $b_{\tau}=0.003$. Note that these values of $k_{\min }$ and $k_{\max }$ correspond, within our scheme, to the most spread choice for the prior distribution of the number of clusters $K$. With regard to the base measure, Figure 3.5 displays the contour plot of the corresponding Kullback-Leibler divergence $\operatorname{KL}\left[g_{0} \| h\right]$. The minimum is attained at $a_{0}=1.778$ and $b_{0}=$ 0.096. Finally, we choose a uniform prior for the thinning parameter $\alpha$, making $a_{\alpha}=1$ and $b_{\alpha}=1$.

The DP-INAR(1) marginal posterior distributions of the parameters $\alpha, \lambda_{3}, \lambda_{18}$, and $\lambda_{96}$ are displayed in Figure 3.6. For this patrol area, the posterior mean of the thinning parameter $\alpha$ is 0.19 . The posterior means of $\lambda_{3}, \lambda_{18}$ and $\lambda_{96}$ are equal to 6.50, 13.61 and 32.01 , respectively, showing that different regimes of innovation rates were captured in the learning process. Figure 3.7 shows both the prior and posterior distributions of the number of clusters $K$. While the prior distribution is reasonably flat in the range 1 to 143, the posterior distribution is concentrated around 7, the posterior mode. Also, Figure 3.8 shows the posterior means of $\lambda_{t}$ as well as the observed counts in patrol area 58. Notice that the estimates of the innovation rates follow, in general, the same pattern as the counts.

The Markov chains in Figures 3.9 and 3.10 indicate that proper mixing is achieved by the Gibbs samplers for both models.

With regard to the forecasting performance within this dataset, Table 3.1 presents the mean absolute deviations of the out-of-sample predictions for the INAR(1), AdINAR(1), and DP-INAR(1), in the 36 patrol areas. In this table, the mean absolute deviations are computed predicting the values of the last 44 months of each time series, using the predictive cross-validation procedure described in Section 3.6.

The results in Table 3.1 show that the AdINAR(1) and the DP-INAR(1) models outperform the INAR(1) model in $75 \%$ of the patrol areas. From the last two columns of the table, we see that the AdINAR(1) model and the DP-INAR(1) model produce substantial relative gains in the mean absolute deviations, with the exception of five areas in which the 

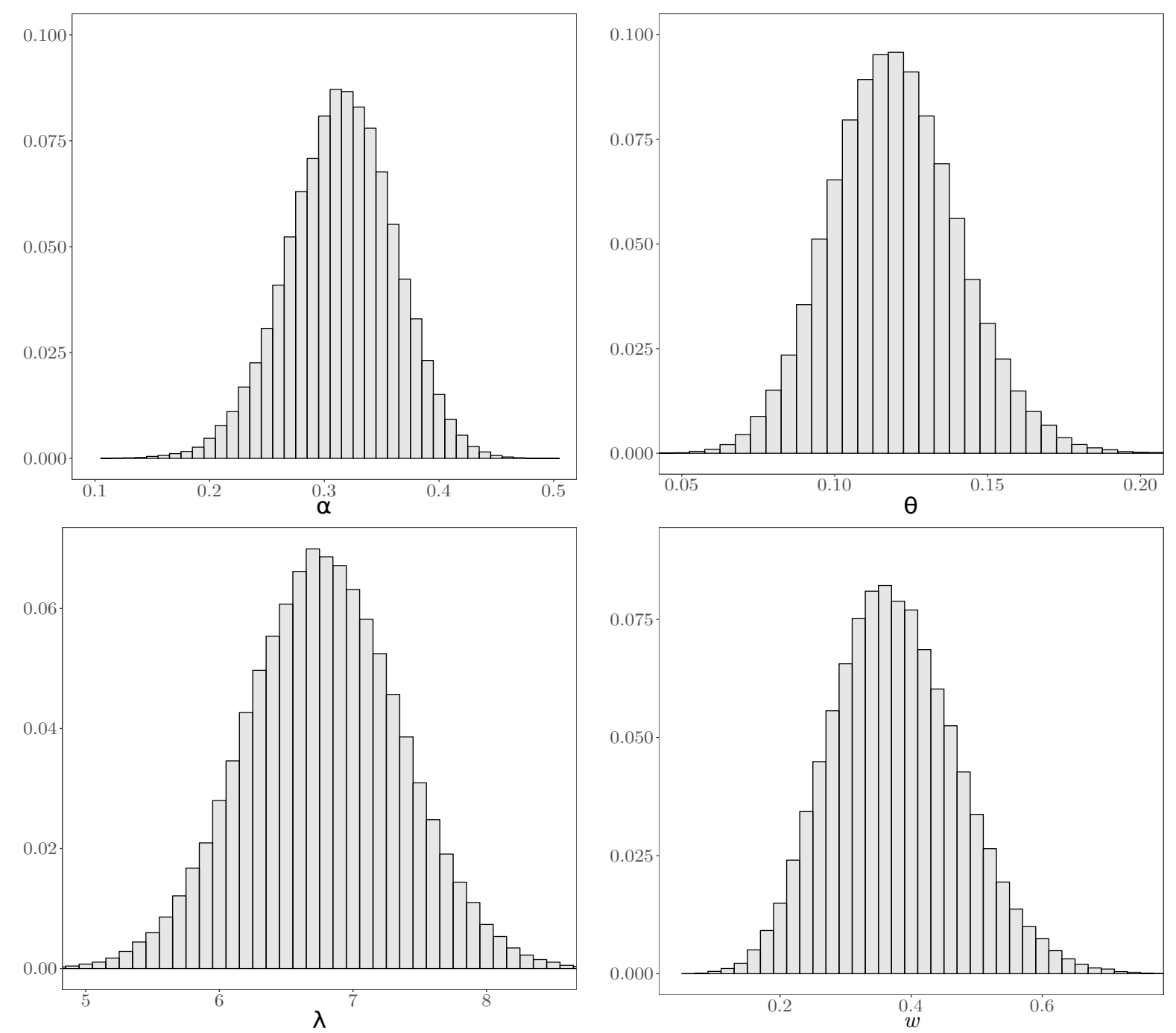

Figure 3.4: Marginal posterior distributions of the AdINAR(1) model parameters $\alpha, \theta, \lambda$, and $w$ for patrol area 58.

INAR(1) performs better, but with smaller relative gains. 


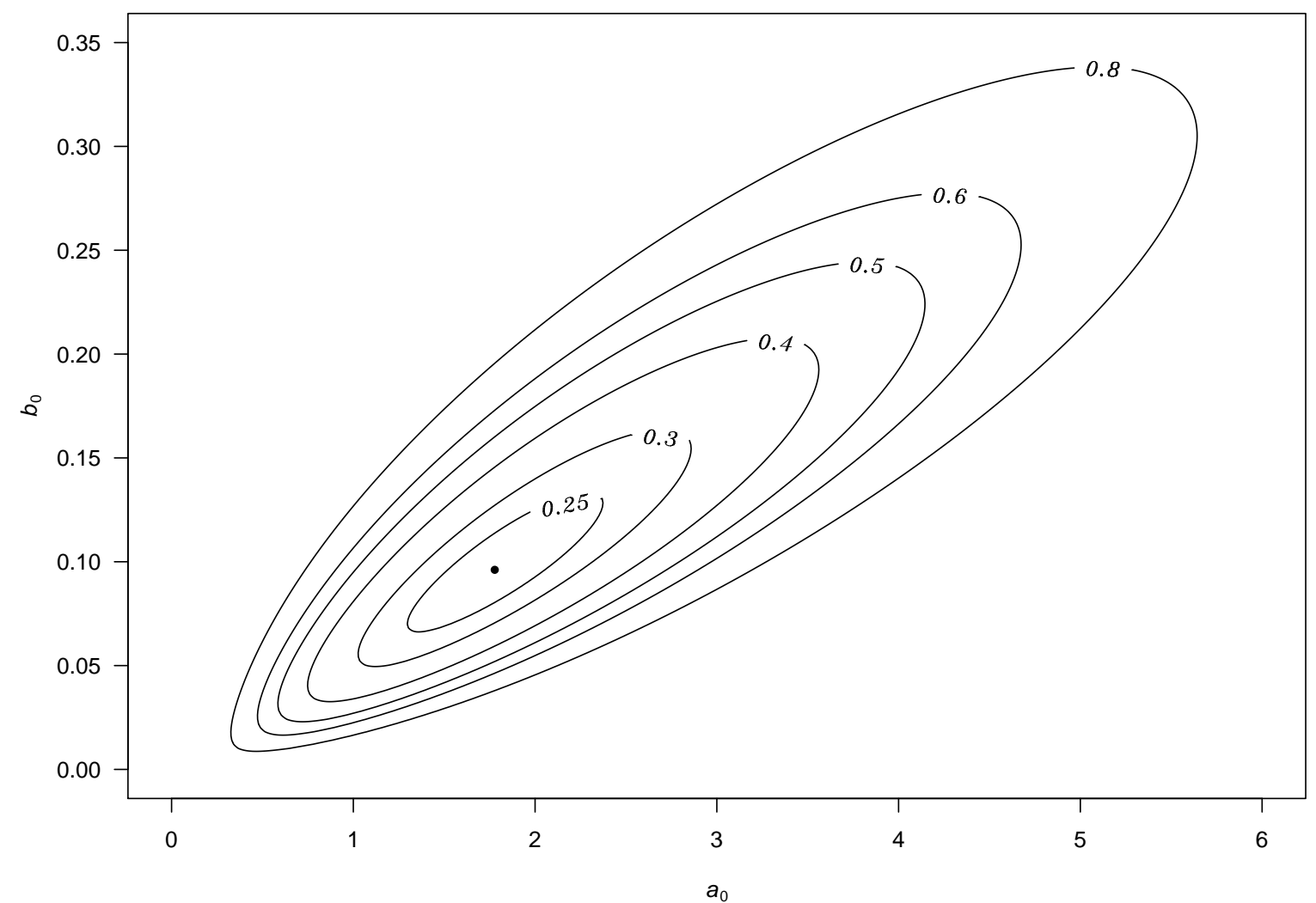

Figure 3.5: Contour plot of the Kullback-Leibler divergence associated with the optimization of the base measure hyperparameters for patrol area 58. 

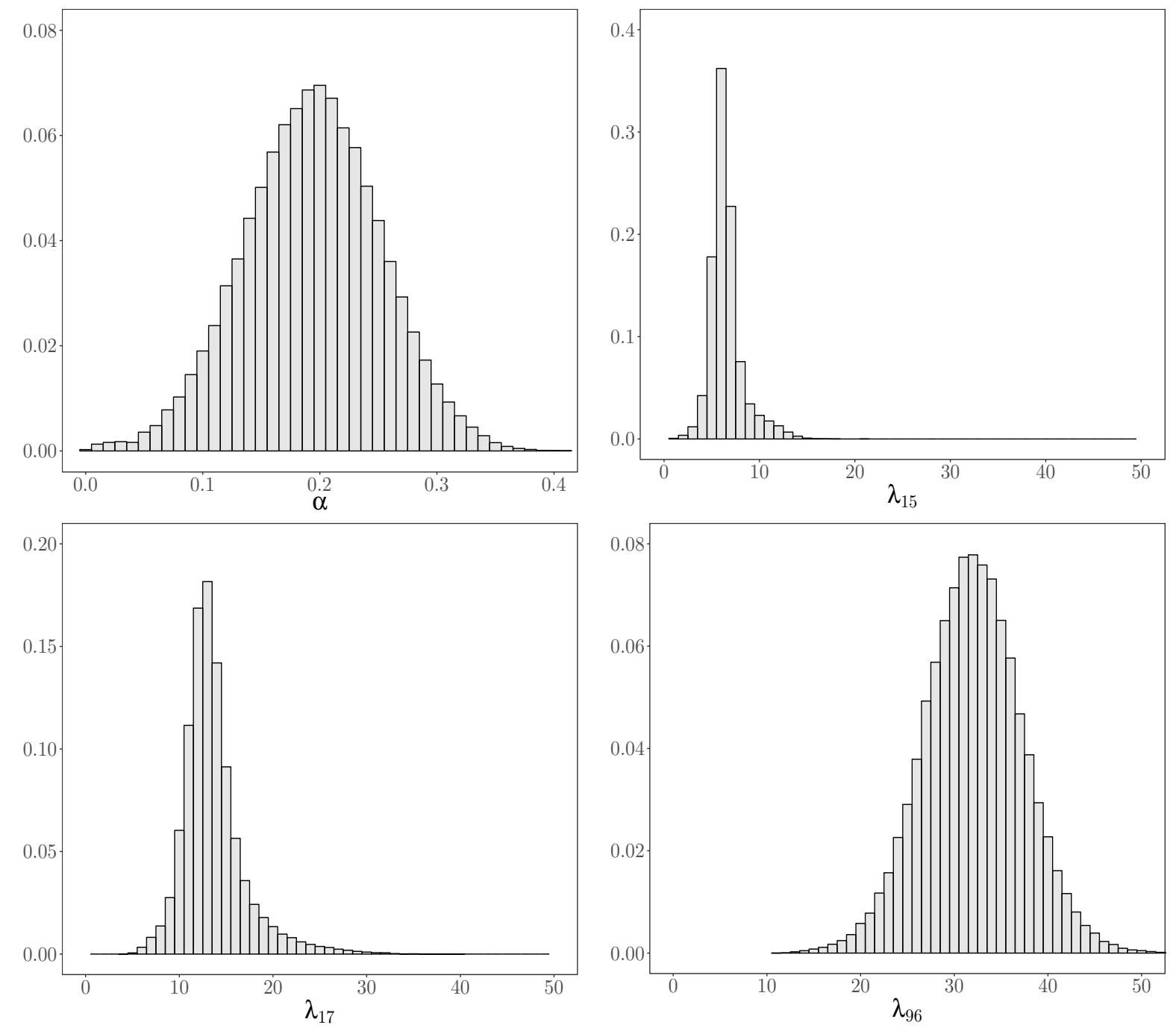

Figure 3.6: Marginal posterior distributions of the DP-INAR(1) model parameters $\alpha, \lambda_{3}, \lambda_{18}$, and $\lambda_{96}$ for patrol area 58. 


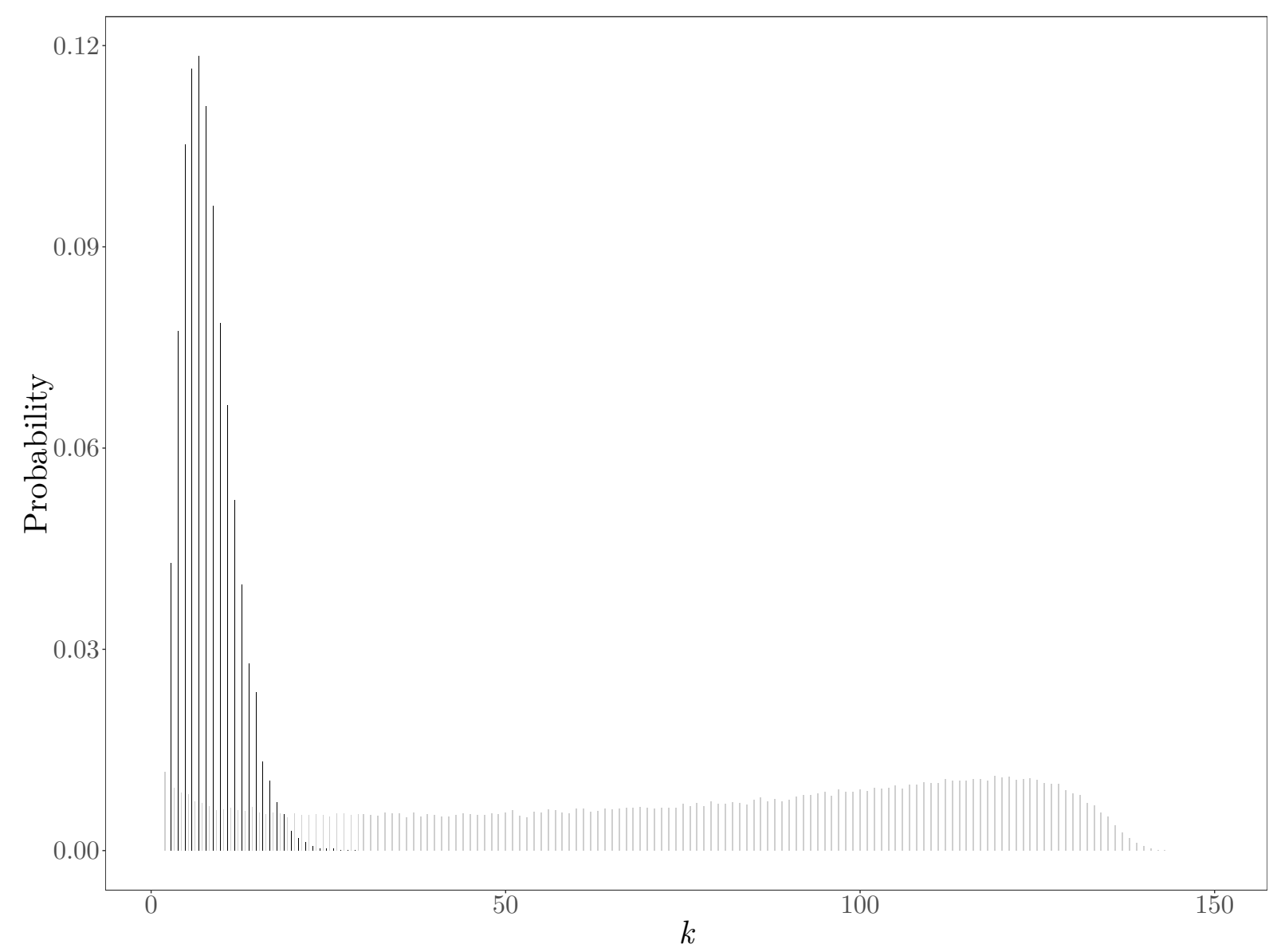

Figure 3.7: DP-INAR(1) model prior and posterior distributions for the number of clusters $K$, in gray and black respectively, for patrol area 58 . 


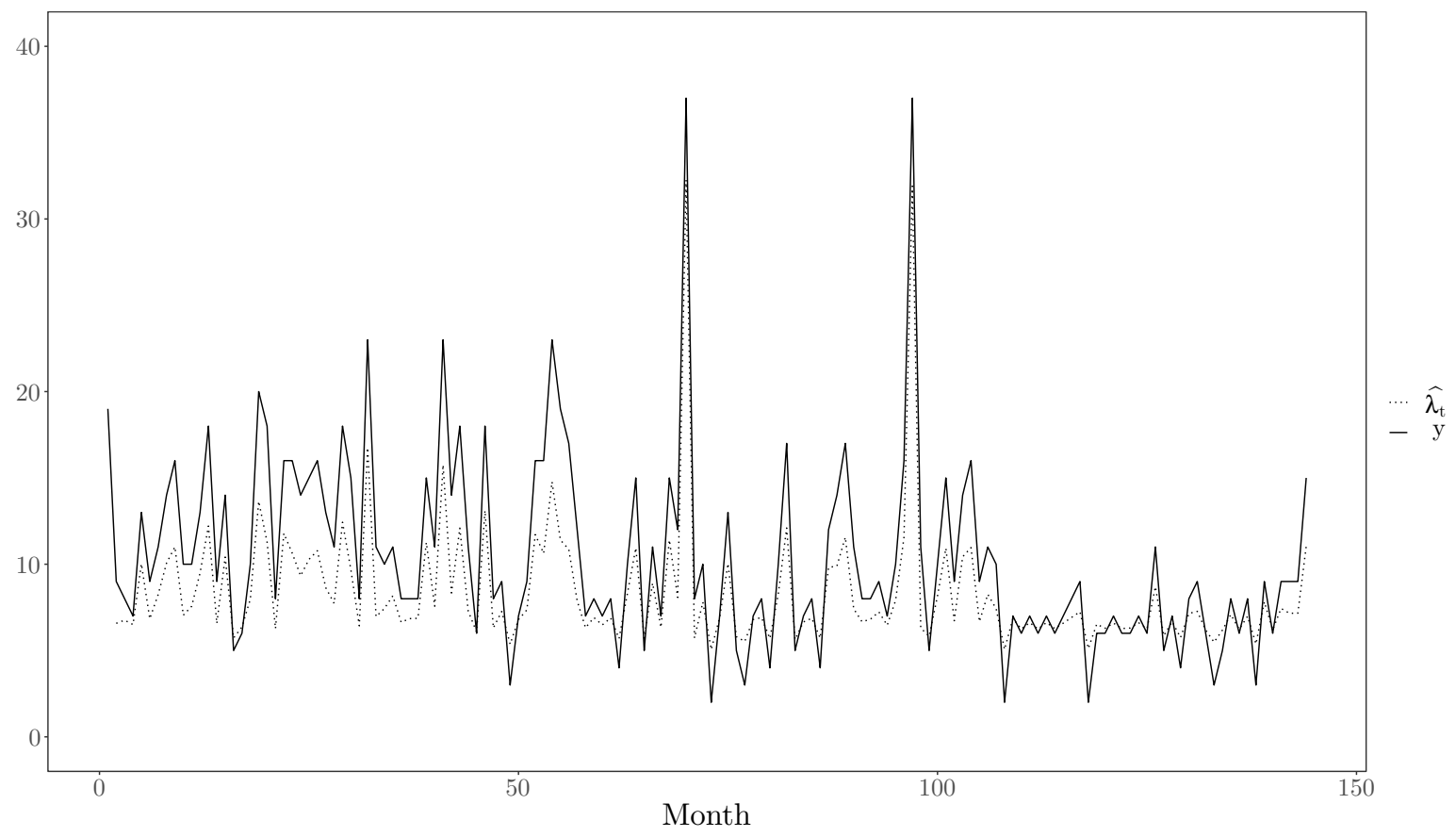

Figure 3.8: Monthly burglary events for patrol area 58 (solid line) and the Bayes estimates of $\lambda_{t}$, $E\left(\lambda_{t} \mid y\right)$, (dashed line) under the DP-INAR(1) model. 

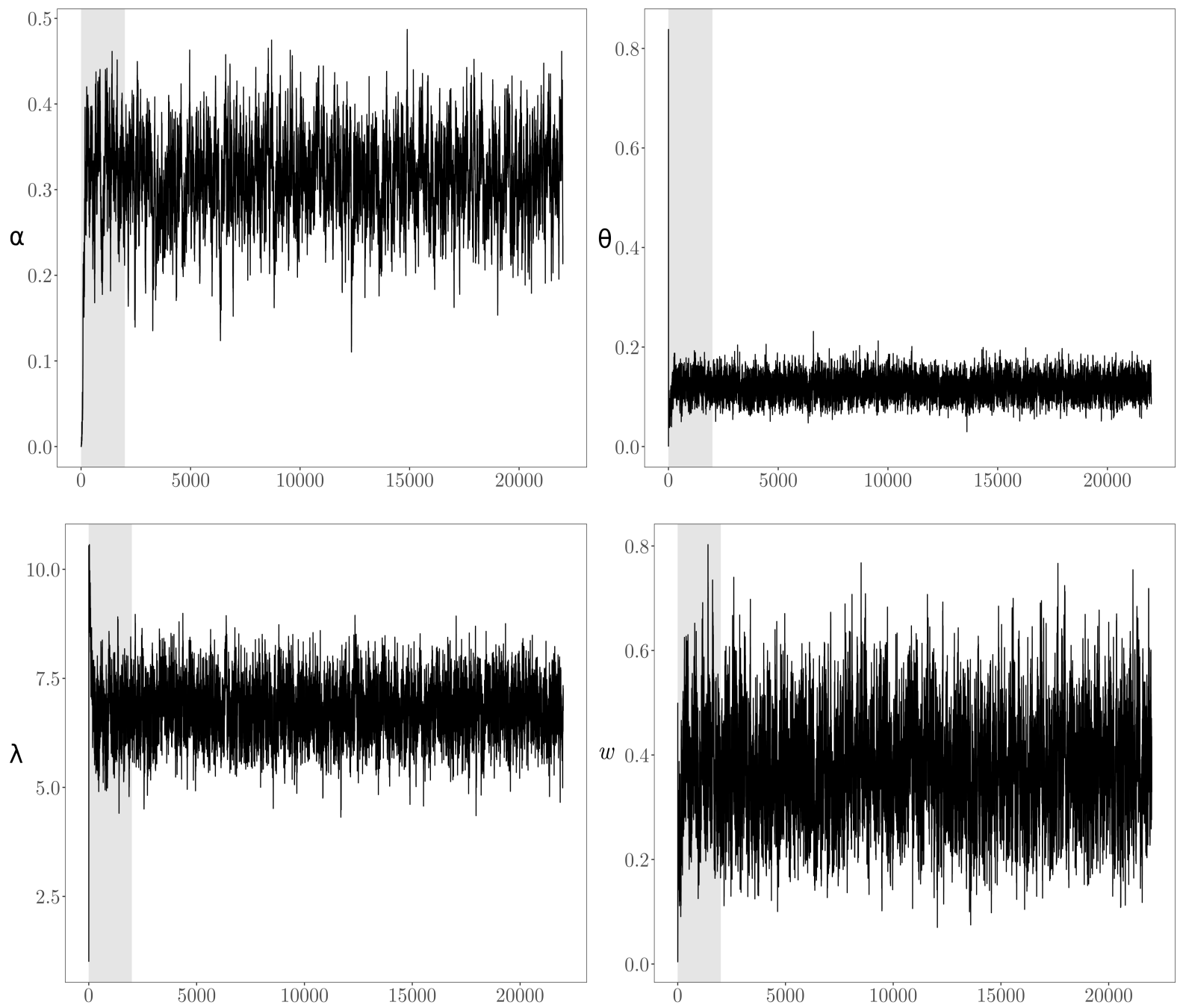

Figure 3.9: Markov chains associated with the AdINAR(1) model marginal posterior distributions of parameters $\alpha, \theta, \lambda$, and $w$ for patrol area 58. The gray rectangles indicate the burn-in periods. 

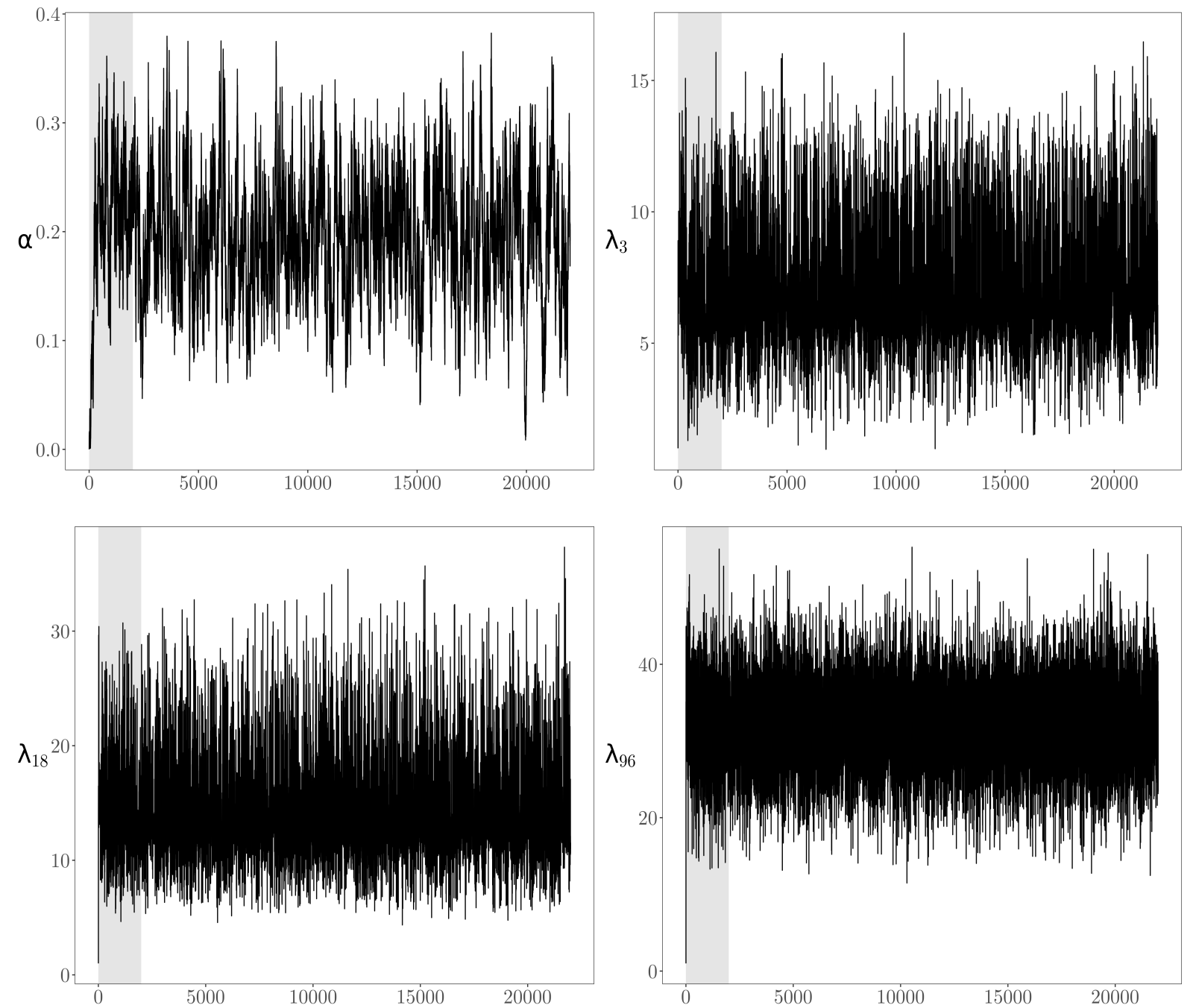

Figure 3.10: Markov chains associated with the DP-INAR(1) model marginal posterior distributions of parameters $\alpha, \lambda_{3}, \lambda_{18}$, and $\lambda_{96}$ for patrol area 58. The gray rectangles indicate the burn-in periods. 
Table 3.1: Mean absolute deviations for the out-of-sample predictions of the INAR(1), AdINAR(1) and DP-INAR(1) models. The last two columns show the relative variations for the AdINAR(1) and $D P-I N A R(1)$ models with respect to the INAR(1) model (lower is better). For each patrol area, the best mean absolute deviation is written in bold face.

\begin{tabular}{|c|c|c|c|c|c|}
\hline Patrol Area & INAR(1) & $\operatorname{AdINAR}(1)$ & DP-INAR(1) & $\Delta_{\text {AdINAR(1) }}$ & $\Delta_{\text {DP-INAR(1) }}$ \\
\hline 11 & $\begin{array}{l}1.209 \\
\end{array}$ & 1.209 & 1.209 & 0.000 & 0.000 \\
\hline 12 & 3.907 & 3.349 & 3.512 & -0.143 & -0.101 \\
\hline 13 & 2.674 & 2.628 & 2.698 & -0.017 & 0.009 \\
\hline 14 & 2.581 & 2.488 & 2.535 & -0.036 & -0.018 \\
\hline 15 & 2.791 & 2.721 & 2.721 & -0.025 & -0.025 \\
\hline 16 & 2.093 & 1.930 & 2.000 & -0.078 & -0.044 \\
\hline 17 & 2.279 & 2.233 & 2.256 & -0.020 & -0.010 \\
\hline 21 & 1.186 & 1.140 & 1.140 & -0.039 & -0.039 \\
\hline 22 & 2.279 & 2.116 & 2.116 & -0.071 & -0.071 \\
\hline 23 & 3.302 & 3.256 & 3.209 & -0.014 & -0.028 \\
\hline 24 & 1.651 & 1.465 & 1.535 & -0.113 & -0.070 \\
\hline 25 & 1.302 & 1.395 & 1.233 & 0.071 & -0.054 \\
\hline 26 & 2.023 & 1.209 & 1.512 & -0.402 & -0.253 \\
\hline 27 & 1.349 & 1.186 & 1.186 & -0.121 & -0.121 \\
\hline 28 & 0.814 & 0.860 & 0.837 & 0.057 & 0.029 \\
\hline 29 & 2.767 & 2.744 & 2.767 & -0.008 & 0.000 \\
\hline 31 & 3.488 & 3.698 & 3.442 & 0.060 & -0.013 \\
\hline 32 & 3.442 & 3.442 & 3.488 & 0.000 & 0.014 \\
\hline 33 & 1.930 & 1.721 & 1.814 & -0.108 & -0.060 \\
\hline 34 & 3.581 & 3.535 & 3.674 & -0.013 & 0.026 \\
\hline 41 & 2.372 & 2.349 & 2.395 & -0.010 & 0.010 \\
\hline 42 & 3.302 & 3.209 & 3.302 & -0.028 & 0.000 \\
\hline 43 & 2.163 & 2.093 & 2.186 & -0.032 & 0.011 \\
\hline 44 & 1.837 & 1.721 & 1.791 & -0.063 & -0.025 \\
\hline 45 & 2.395 & 2.326 & 2.395 & -0.029 & 0.000 \\
\hline 46 & 2.744 & 2.744 & 2.628 & 0.000 & -0.042 \\
\hline 47 & 2.302 & 2.465 & 2.256 & 0.071 & -0.020 \\
\hline 51 & 2.860 & 3.093 & 2.930 & 0.081 & 0.024 \\
\hline 52 & 3.814 & 3.977 & 3.930 & 0.043 & 0.030 \\
\hline 53 & 2.837 & 2.930 & 2.884 & 0.033 & 0.016 \\
\hline 54 & 2.884 & 2.558 & 2.535 & -0.113 & -0.121 \\
\hline 55 & 4.512 & 5.419 & 4.884 & 0.201 & 0.082 \\
\hline 56 & 2.093 & 1.884 & 1.930 & -0.100 & -0.078 \\
\hline 57 & 1.977 & 2.047 & 1.977 & 0.035 & 0.000 \\
\hline 58 & 2.977 & 2.372 & 2.512 & -0.203 & -0.156 \\
\hline
\end{tabular}




\section{Chapter 4}

\section{A higher order semi-parametric model for time series of counts}

In this chapter, we examine issues of prior sensitivity in a proposed semi-parametric generalization of the INAR model, in which the innovation rates are clustered with the help of a Pitman-Yor process, a generalization of the Dirichlet process, placed at the top of the model hierarchy. First, we extend the first-order autoregressive structure to a general order, which is a Bayesian counterpart to the $\operatorname{INAR}(p)$ model, firstly proposed by Du and Li [1991]. Second, we propose a graphical criterion that guides the specification of the hyperparameters of the Pitman-Yor process base measure. We show how the discount and concentration parameters interact with the chosen base measure to yield a gain in terms of the robustness of the inferential results. The forecasting performance of the model is exemplified in the analysis of a time series of worldwide earthquake events, for which the new model outperforms the original $\operatorname{INAR}(p)$ model.

\subsection{Generalizing the autoregressive order}

We begin by generalizing the original $\operatorname{INAR}(p)$ model of $\mathrm{Du}$ and Li [1991] as follows.

Let $\left\{Y_{t}\right\}_{t \geq 1}$ be an integer-valued time series, and, for some integer $p \geq 1$, let the innovations $\left\{Z_{t}\right\}_{t \geq p+1}$, given positive parameters $\left\{\lambda_{t}\right\}_{t \geq p+1}$, be a sequence of conditionally independent Poisson $\left(\lambda_{t}\right)$ random variables. For a given vector of parameters $\alpha=\left(\alpha_{1}, \ldots, \alpha_{p}\right) \in$ $[0,1]^{p}$, let $\mathscr{F}_{i}=\left\{B_{i j}(t): j \geq 0, t \geq 2\right\}$ be a family of conditionally independent and identically distributed Bernoulli $\left(\alpha_{i}\right)$ random variables. For $i \neq k$, suppose that $\mathscr{F}_{i}$ and $\mathscr{F}_{k}$ are conditionally independent, given $\alpha$. Furthermore, given all the parameters, assume that the innovations $\left\{Z_{t}\right\}_{t \geq p+1}$ and the families $\mathscr{F}_{1}, \ldots, \mathscr{F}_{p}$ are conditionally independent. The 
generalized $\operatorname{INAR}(p)$ model is defined by the functional relation

$$
Y_{t}=\alpha_{1} \circ Y_{t-1}+\cdots+\alpha_{p} \circ Y_{t-p}+Z_{t},
$$

for $t \geq p+1$, in which $\circ$ denotes the binomial thinning operator, defined by $\alpha_{i} \circ Y_{t-i}=$ $\sum_{j=1}^{Y_{t-i}} B_{i j}(t)$, if $Y_{t-i}>0$, and $\alpha_{i} \circ Y_{t-i}=0$, if $Y_{t-i}=0$. In the homogeneous case, when all the $\lambda_{t}$ 's are assumed to be equal, we recover the original $\operatorname{INAR}(p)$ model.

When $p=1$, this model can be interpreted as specifying a birth-and-death process, in which, at epoch $t$, the number of cases $Y_{t}$ is equal to the new cases $Z_{t}$ plus the cases that survived from the previous epoch; the role of the binomial thinning operator being to remove a random number of the $Y_{t-1}$ cases present at the previous epoch $t-1$ (see Dion et al. [1995] for an interpretation of the order $p$ case as a birth-and-death process with immigration).

Let $y=\left(y_{1}, \ldots, y_{T}\right)$ denote the values of an observed time series. For simplicity, we assume that $Y_{1}=y_{1}, \ldots, Y_{p}=y_{p}$ with probability one. The joint distribution of $Y_{1}, \ldots, Y_{T}$, given parameters $\alpha$ and $\lambda=\left(\lambda_{p+1}, \ldots, \lambda_{T}\right)$, can be factored as

$$
\operatorname{Pr}\left\{Y_{1}=y_{1}, \ldots, Y_{T}=y_{T} \mid \alpha, \lambda\right\}=\prod_{t=p+1}^{T} \operatorname{Pr}\left\{Y_{t}=y_{t} \mid Y_{t-1}=y_{t-1}, \ldots, Y_{t-p}=y_{t-p}, \alpha, \lambda_{t}\right\}
$$

Since, with probability one, $\alpha_{i} \circ Y_{t-i} \leq Y_{t-i}$ and $Z_{t} \geq 0$, the likelihood function of the generalized $\operatorname{INAR}(p)$ model is given by

$$
\begin{array}{r}
L_{y}(\alpha, \lambda)=\prod_{t=p+1}^{T} \sum_{m_{1, t}=0}^{\min \left\{y_{t}, y_{t-1}\right\}} \cdots \sum_{m_{p, t}=0}^{\min \left\{y_{t}-\sum_{j=1}^{p-1} m_{j, t}, y_{t-p}\right\}}\left(\prod_{i=1}^{p}\left(\begin{array}{c}
y_{t-i} \\
m_{i, t}
\end{array}\right) \alpha_{i}^{m_{i, t}}\left(1-\alpha_{i}\right)^{y_{t-i}-m_{i, t}}\right) \times \\
\left(\frac{e^{-\lambda_{t}} \lambda_{t}^{y_{t}-\sum_{j=1}^{p} m_{j, t}}}{\left(y_{t}-\sum_{j=1}^{p} m_{j, t}\right) !}\right) .
\end{array}
$$

For some epoch $t$ and $i=1, \ldots, p$, suppose that we could observe the values of the latent maturations $M_{i, t}$. Postulate that

$$
M_{i, t} \mid Y_{t-i}=y_{t-i}, \alpha_{i} \sim \operatorname{Binomial}\left(y_{t-i}, \alpha_{i}\right),
$$


so that the conditional probability function of $M_{i, t}$ is given by

$$
\begin{aligned}
p\left(m_{i, t} \mid y_{t-i}, \alpha_{i}\right) & =\operatorname{Pr}\left\{M_{i, t}=m_{i, t} \mid Y_{t-i}=y_{t-i}, \alpha_{i}\right\} \\
& =\left(\begin{array}{c}
y_{t-i} \\
m_{i, t}
\end{array}\right) \alpha_{i}^{m_{i, t}}\left(1-\alpha_{i}\right)^{y_{t-i}-m_{i, t}} \mathbb{I}_{\left\{0, \ldots, y_{t-i}\right\}}\left(m_{i, t}\right) .
\end{aligned}
$$

Furthermore, suppose that

$$
\begin{aligned}
p\left(y_{t} \mid m_{1, t}, \ldots, m_{p, t}, \lambda_{t}\right) & =\operatorname{Pr}\left\{Y_{t}=y_{t} \mid M_{1, t}=m_{1, t}, \ldots, M_{p, t}=m_{p, t}, \lambda_{t}\right\} \\
& =\frac{e^{-\lambda_{t}} \lambda_{t}^{y_{t}-\sum_{j=1}^{p} m_{j, t}}}{\left(y_{t}-\sum_{j=1}^{p} m_{j, t}\right) !} \mathbb{I}_{\left\{\sum_{j=1}^{p} m_{j, t}, \sum_{j=1}^{p} m_{j, t}+1, \ldots\right\}}\left(y_{t}\right) .
\end{aligned}
$$

Using the law of total probability and the product rule, we have that

$$
\begin{aligned}
p\left(y_{t} \mid y_{t-1}, \ldots, y_{t-p}, \alpha, \lambda_{t}\right) & =\sum_{m_{1, t}=0}^{y_{t-1}} \ldots \sum_{m_{p, t}=0}^{y_{t-p}} p\left(y_{t}, m_{1, t}, \ldots, m_{p, t} \mid y_{t-1}, \ldots, y_{t-p}, \alpha, \lambda_{t}\right) \\
& =\sum_{m_{1, t}=0}^{y_{t-1}} \cdots \sum_{m_{p, t}=0}^{y_{t-p}} p\left(y_{t} \mid m_{1, t}, \ldots, m_{p, t}, \lambda_{t}\right) \times \prod_{i=1}^{p} p\left(m_{i, t} \mid y_{t-i}, \alpha_{i}\right) .
\end{aligned}
$$

Since

$$
\begin{aligned}
\mathbb{I}_{\left\{\sum_{j=1}^{p} m_{j, t}, \sum_{j=1}^{p} m_{j, t}+1, \ldots\right\}}\left(y_{t}\right) & =\mathbb{I}_{\left\{0, \ldots, y_{t}\right\}}\left(\sum_{j=1}^{p} m_{j, t}\right) \\
& =\mathbb{I}_{\left\{0, \ldots, y_{t}\right\}}\left(m_{1, t}\right) \times \cdots \times \mathbb{I}_{\left\{0, \ldots, y_{t}-\sum_{j=1}^{p-1} m_{j, t}\right\}}\left(m_{p, t}\right)
\end{aligned}
$$

and

$$
\mathbb{I}_{\left\{\sum_{j=1}^{p} m_{j, t}, \sum_{j=1}^{p} m_{j, t}+1, \ldots\right\}}\left(y_{t}\right) \times \mathbb{I}_{\left\{0, \ldots, y_{t-i}\right\}}\left(m_{i, t}\right)=\mathbb{I}_{\left\{0,1, \ldots, \min \left\{y_{t}-\sum_{j \neq i} m_{j, t}, y_{t-i}\right\}\right\}}\left(m_{i, t}\right)
$$

we recover the original likelihood of the generalized $\operatorname{INAR}(p)$, showing that the introduction of the latent maturations $M_{i, t}$ with the specified distributions is a valid data augmentation scheme (see Tanner and Wong [1987], Van Dyk and Meng [2001] for a general discussion of data augmentation techniques).

In the next section, we review the needed definitions and properties of the Pitman-Yor process. 


\subsection{Pitman-Yor process}

Let the random probability measure $\mathbb{G} \sim \operatorname{DP}\left(\tau, G_{0}\right)$ be a Dirichlet process [Ferguson, 1973, Hjort et al., 2010, Schervish, 1995] with concentration parameter $\tau$ and base measure $G_{0}$. If the random variables $X_{1}, \ldots, X_{n}$, given $\mathbb{G}=G$, are conditionally independent and identically distributed as $G$, then it follows that

$$
\operatorname{Pr}\left\{X_{n+1} \in B \mid X_{1}=x_{1}, \ldots, X_{n}=x_{n}\right\}=\frac{\tau}{\tau+n} G_{0}(B)+\frac{1}{\tau+n} \sum_{i=1}^{n} I_{B}\left(x_{i}\right),
$$

for every Borel set $B$. If we imagine the sequential generation of the $X_{i}$ 's, for $i=1, \ldots, n$, the former expression shows that a value is generated anew from $G_{0}$ with probability proportional to $\tau$, or we repeat one the previously generated values with probability proportional to its multiplicity. Therefore, almost surely, realizations of a Dirichlet process are discrete probability measures, perhaps with denumerable infinite support, depending on the nature of $G_{0}$. Also, this data generating process, known as the Pólya-Blackwell-MacQueen urn, implies that the $X_{i}$ 's are "softly clustered", in the sense that in one realization of the process the elements of a subset of the $X_{i}$ 's may have exactly the same value.

The Pitman-Yor process [Pitman et al., 2002] is a generalization of the Dirichlet process which results in a model with added flexibility. Essentially, the Pitman-Yor process modifies the expression of the probability associated with the Pólya-Blackwell-MacQueen urn introducing a new parameter so that the posterior predictive probability becomes

$$
\operatorname{Pr}\left\{X_{n+1} \in B \mid X_{1}=x_{1}, \ldots, X_{n}=x_{n}\right\}=\frac{\tau+k \sigma}{\tau+n} G_{0}(B)+\frac{1}{\tau+n} \sum_{i=1}^{n}\left(1-\frac{\sigma}{\nu_{i}}\right) I_{B}\left(x_{i}\right),
$$

in which $0 \leq \sigma<1$ is the discount parameter, $\tau>-\sigma, k$ is the number of distinct elements in $\left\{X_{1}, \ldots, X_{n}\right\}$, and $\nu_{i}$ is the number of elements in $\left\{X_{1}, \ldots, X_{n}\right\}$ which are equal to $X_{i}$, for $i=1, \ldots, n$. It is well known that $\mathrm{E}[\mathbb{G}(B)]=G_{0}(B)$ and

$$
\operatorname{Var}[\mathbb{G}(B)]=\left(\frac{1-\sigma}{\tau+1}\right) G_{0}(B)\left(1-G_{0}(B)\right)
$$

for every Borel set $B$. Hence, $\mathbb{G}$ is centered on the base probability measure $G_{0}$, while $\tau$ and $\sigma$ control the concentration of $\mathbb{G}$ around $G_{0}$. We use the notation $\mathbb{G} \sim \operatorname{PY}\left(\tau, \sigma, G_{0}\right)$. When $\sigma=0$, we recover the Dirichlet process as a special case.

Pitman et al. [2002] derived the conditional distribution of the number of clusters $K$ 


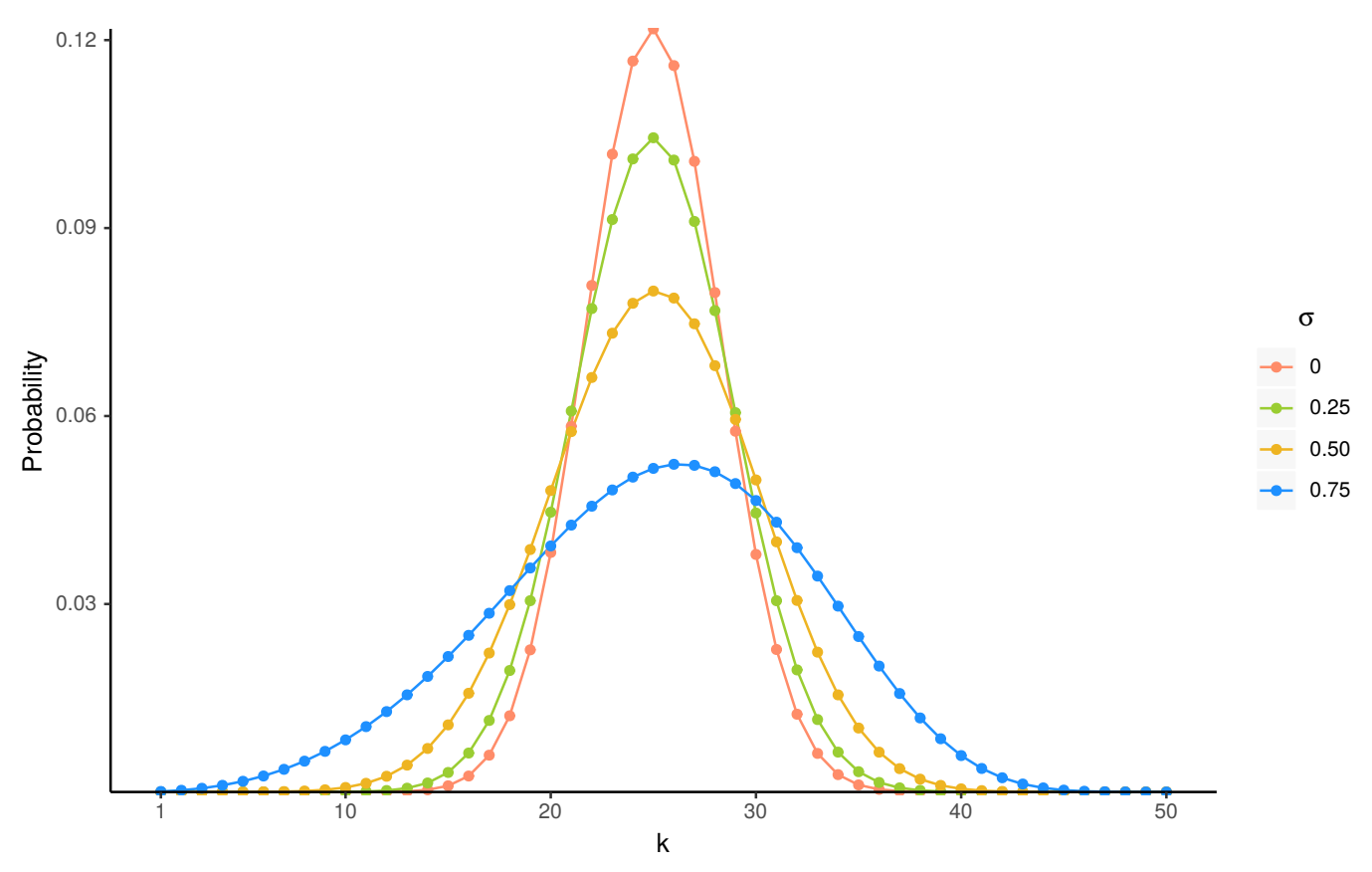

Figure 4.1: Prior distributions of $K$ in the Pitman-Yor process, with $\sigma \in\{0,0.25,0.5,0.75\}, n=50$ and $\tau$ fixed such that $E(K)=25$.

(the number of distinct $X_{i}^{\prime}$ 's), given both the concentration parameter $\tau$ and the discount parameter $\sigma$, as

$$
\operatorname{Pr}\{K=k \mid \tau, \sigma\}=\frac{\prod_{i=1}^{k-1}(\tau+i \sigma)}{\sigma^{k} \times(\tau+1)_{n-1}} \times \mathscr{C}(n, k ; \sigma),
$$

in which $(x)_{n}=\Gamma(x+n) / \Gamma(x)$ is the rising factorial and $\mathscr{C}(n, k ; \sigma)$ is the generalized factorial coefficient [Lijoi et al., 2007]. Figure 4.1 displays these distributions for $\sigma \in\{0,0.25,0.5,0.75\}$, $n=50$, and $\tau$ fixed such that $E(K)=25$. Notice that as $\sigma$ gets larger, the process induces less informative prior distributions for $K$.

In the next section, we use a Pitman-Yor process to model the distribution of the innovation rates in the generalized $\operatorname{INAR}(p)$ model.

\subsection{PY-INAR $(p)$ model}

The PY-INAR $(p)$ model is as a hierarchical extension of the generalized $\operatorname{INAR}(p)$ model defined in Section 3.1. Given a random measure $\mathbb{G} \sim \operatorname{PY}\left(\tau, \sigma, G_{0}\right)$, in which $G_{0}$ is a $\operatorname{Gamma}\left(a_{0}, b_{0}\right)$ distribution, let the innovation rates $\lambda_{p+1}, \ldots, \lambda_{T}$ be conditionally independent and identically distributed with distribution $\operatorname{Pr}\left\{\lambda_{t} \in B \mid \mathbb{G}=G\right\}=G(B)$.

To complete the PY-INAR $(p)$ model, we need to specify the form of the prior distribution for the vector of thinning parameters $\alpha=\left(\alpha_{1}, \ldots, \alpha_{p}\right)$. By comparison with stan- 
dard results from the theory of the $\operatorname{AR}(p)$ model [Hamilton, 1994]. Du and Li [1991] found that in the $\operatorname{INAR}(p)$ model the constraint $\sum_{i=1}^{p} \alpha_{i}<1$ must be fulfilled to guarantee the non-explosiveness of the process. In their Bayesian analysis of the $\operatorname{INAR}(p)$ model, Neal and Kypraios [2015] considered independent beta distributions for the $\alpha_{i}{ }^{\prime}$ s. Unfortunately, this choice is problematic. For example, in the particular case when the $\alpha_{i}$ 's have independent uniform distributions, it is possible to show that $\operatorname{Pr}\left\{\sum_{i=1}^{p} \alpha_{i}<1\right\}=1 / p$ !, implying that we would be concentrating most of the prior mass on the explosive region even for moderate values of the model order $p$ (e.g.: this probability is 0.008 when $p=5$ ). We circumvent this problem using a prior distribution for $\alpha$ that places all of its mass on the nonexplosive region and still allows us to derive the full conditional distributions of the $\alpha_{i}$ 's in simple closed form. Specifically, we take the prior distribution of $\alpha$ to be a Dirichlet distribution with hyperparameters $\left(a_{1}, \ldots, a_{p} ; a_{p+1}\right)$, and corresponding density

$$
\pi(\alpha)=\frac{\Gamma\left(\sum_{i=1}^{p+1} a_{i}\right)}{\prod_{i=1}^{p+1} \Gamma\left(a_{i}\right)} \prod_{i=1}^{p+1} \alpha_{i}^{a_{i}-1}
$$

in which $a_{i}>0$, for $i=1, \ldots, p+1$, and $\alpha_{p+1}=1-\sum_{i=1}^{p} \alpha_{i}$.

Let $m=\left\{m_{i, t}: i=1, \ldots, p, t=p+1, \ldots, T\right\}$ denote the set of all maturations, and let $\mu_{\mathbb{G}}$ be the distribution of $\mathbb{G}$. Our strategy to derive the full conditional distributions of the model parameters and latent variables is to consider the marginal distribution

$$
\begin{aligned}
p(y, m, \alpha, \lambda)= & \int p(y, m, \alpha, \lambda \mid G) d \mu_{\mathbb{G}}(G) \\
= & \left\{\prod_{t=p+1}^{T} p\left(y_{t} \mid m_{1, t}, \ldots, m_{p, t}, \lambda_{t}\right) \prod_{i=1}^{p} p\left(m_{i, t} \mid y_{t-i}, \alpha_{i}\right)\right\} \\
& \times \pi(\alpha) \times \int \prod_{t=p+1}^{T} p\left(\lambda_{t} \mid G\right) d \mu_{\mathbb{G}}(G) .
\end{aligned}
$$

From this expression, using the results in Section 4.2, the derivation of the full conditional distributions is straightforward. In the following expressions, the symbol $\propto$ denotes proportionality up to a suitable normalization factor, and the label "all others" designate the observed counts $y$ and all the other latent variables and model parameters, with the exception of the one under consideration.

Let $\lambda_{\backslash t}$ denote the set $\left\{\lambda_{p+1}, \ldots, \lambda_{T}\right\}$ with the element $\lambda_{t}$ removed. Then, for $t=p+$ 
$1, \ldots, T$, we have

$\lambda_{t} \mid$ all others $\sim w_{t} \times \operatorname{Gamma}\left(a_{0}+y_{t}-\sum_{i=1}^{p} m_{i, t}, b_{0}+1\right)+\sum_{r \neq t}\left(1-\frac{\sigma}{\nu_{r}}\right) \lambda_{r}^{y_{t}-\sum_{i=1}^{p} m_{i, t}} e^{-\lambda_{r}} \delta_{\left\{\lambda_{r}\right\}}$, in which the weight

$$
w_{t}=\frac{\left(\tau+k_{\backslash t} \sigma\right) \times b_{0}^{a_{0}} \times \Gamma\left(a_{0}+y_{t}-\sum_{i=1}^{p} m_{i, t}\right)}{\Gamma\left(a_{0}\right) \times\left(b_{0}+1\right)^{a_{0}+y_{t}-\sum_{i=1}^{p} m_{i, t}}},
$$

$\nu_{r}$ is the number of elements in $\lambda_{\backslash t}$ that are equal to $\lambda_{r}$, and $k_{\backslash t}$ is the number of distinct elements in $\lambda_{\backslash t}$. In this mixture, we suppressed the normalization constant that makes all weights add up to one.

Making the choice $a_{p+1}=1$, we have

$$
\alpha_{i} \mid \text { all others } \sim \operatorname{TBeta}\left(a_{i}+\sum_{t=p+1}^{T} m_{i, t}, 1+\sum_{t=p+1}^{T}\left(y_{t-i}-m_{i, t}\right) ; 1-\sum_{j \neq i} \alpha_{j}\right)
$$

for $i=1, \ldots, p$, in which TBeta denotes the right truncated Beta distribution with support $\left(0,1-\sum_{j \neq i}^{p} \alpha_{j}\right)$.

For the latent maturations, we find

$$
\begin{aligned}
p\left(m_{i, t} \mid \text { all others }\right) \propto & \frac{1}{\left(m_{i, t}\right) !\left(y_{t}-\sum_{j=1}^{p} m_{j, t}\right) !\left(y_{t-i}-m_{i, t}\right) !}\left(\frac{\alpha_{i}}{\lambda_{t}\left(1-\alpha_{i}\right)}\right)^{m_{i, t}} \\
& \times \mathbb{I}_{\left\{0,1, \ldots, \min \left\{y_{t}-\sum_{j \neq i} m_{j, t}, y_{t-i}\right\}\right\}}\left(m_{i, t}\right) .
\end{aligned}
$$

As in the DP-INAR(1) model, we explore the posterior distribution of this model, by building a Gibbs sampler [Gamerman and Lopes, 2006] using these full conditional distributions. Similarly, we can improve mixing by resampling simultaneously the values of all $\lambda_{t}$ 's inside the same cluster at the end of each iteration of the Gibbs sampler. Letting $\left(\lambda_{1}^{*}, \ldots, \lambda_{k}^{*}\right)$ be the $k$ unique values among $\left(\lambda_{p+1}, \ldots, \lambda_{T}\right)$, define the number of occupants of cluster $j$ by $n_{j}=\sum_{t=p+1}^{T} \mathbb{I}_{\left\{\lambda_{j}^{*}\right\}}\left(\lambda_{t}\right)$. It follows that

$$
\lambda_{j}^{*} \mid \text { all others } \sim \text { Gamma }\left(a_{0}+\sum_{t=p+1}^{T}\left(y_{t}-\sum_{i=1}^{p} m_{i, t}\right) \cdot \mathbb{I}_{\left\{\lambda_{j}^{*}\right\}}\left(\lambda_{t}\right), b_{0}+n_{j}\right) .
$$

for $j=1, \ldots, k$. We update the values of all $\lambda_{t}$ 's inside each cluster by the corresponding $\lambda_{j}^{*}$ using this distribution. 


\subsection{Prior sensitivity}

As it is often the case for Bayesian models with nonparametric components, a choice of the prior parameters of the PY-INAR $(p)$ model which yields robustness of the posterior distribution is nontrivial [Canale and Prünster, 2017]. The first aspect to be considered is the fact that the support of the base measure $G_{0}$ plays a crucial role in the determination of the posterior distribution of the number of clusters $K$. This can be seen directly by inspecting the form of the full conditional distributions. Recalling that $G_{0}$ is a gamma distribution with mean $a_{0} / b_{0}$ and variance $a_{0} / b_{0}^{2}$, note that in the expression of the $\lambda_{t}$ full conditional distribution the probability of generating, on each iteration of the Gibbs sampler, a value for $\lambda_{t}$ anew from $G_{0}$ is proportional to

$$
\frac{\left(\tau+k_{\backslash t} \sigma\right) \times b_{0}^{a_{0}} \times \Gamma\left(y_{t}-m_{t}+a_{0}\right)}{\Gamma\left(a_{0}\right)\left(b_{0}+1\right)^{y_{t}-m_{t}+a_{0}}} .
$$

Therefore, supposing that all the other terms are fixed, if we concentrate the support of $G_{0}$ by making $b_{0} \rightarrow \infty$, this probability decreases to zero, as follows

$$
\begin{array}{r}
\frac{\left(\tau+k_{\backslash t} \sigma\right) \times\left(b_{0}^{\left(G_{0}\right)}\right)^{a_{0}^{\left(G_{0}\right)}} \times \Gamma\left(y_{t}-m_{t}+a_{0}^{\left(G_{0}\right)}\right)}{\Gamma\left(a_{0}^{\left(G_{0}\right)}\right)\left(b_{0}^{\left(G_{0}\right)}+1\right)^{y_{t}-m_{t}+a_{0}^{(G)}}}<\frac{\left(\tau+k_{\backslash t} \sigma\right) \times \Gamma\left(y_{t}-m_{t}+a_{0}^{\left(G_{0}\right)}\right)}{\Gamma\left(a_{0}^{\left(G_{0}\right)}\right)} \\
\times\left(\frac{1}{b_{0}^{\left(G_{0}\right)}}\right)^{y_{t}-m_{t}} \rightarrow 0,
\end{array}
$$

as $b_{0}^{\left(G_{0}\right)} \rightarrow \infty$, if $y_{t}-m_{t}>0$, and for every $a_{0}^{\left(G_{0}\right)}>0, \tau>-\sigma$.

This is not problematic, because it is hardly the case that we want to impose such a drastic restriction on the support of $G_{0}$. The behavior in the other direction is more revealing, since taking $b_{0} \downarrow 0$, in order to spread the support of $G_{0}$, also makes the limit of this probability to be zero, as follows

$$
\begin{aligned}
& \frac{\left(\tau+k_{\backslash t} \sigma\right) \times\left(b_{0}^{\left(G_{0}\right)}\right)^{a_{0}^{\left(G_{0}\right)}} \times \Gamma\left(y_{t}-m_{t}+a_{0}^{\left(G_{0}\right)}\right)}{\Gamma\left(a_{0}^{\left(G_{0}\right)}\right)\left(b_{0}^{\left(G_{0}\right)}+1\right)^{y_{t}-m_{t}+a_{0}^{\left(G_{0}\right)}}}<\frac{\left(\tau+k_{\backslash t} \sigma\right) \times \Gamma\left(y_{t}-m_{t}+a_{0}^{\left(G_{0}\right)}\right)}{\Gamma\left(a_{0}^{\left(G_{0}\right)}\right)} \\
& \times\left(\frac{b_{0}^{\left(G_{0}\right)}}{b_{0}^{\left(G_{0}\right)}+1}\right)^{a_{0}^{\left(G_{0}\right)}} \rightarrow 0
\end{aligned}
$$

as $b_{0}^{\left(G_{0}\right)} \rightarrow 0$, for every $a_{0}^{\left(G_{0}\right)}>0, \tau>-\sigma, y_{t}$, and $m_{t}$. Due to this behavior, we need to 
establish a criterion to choose the hyperparameters of $G_{0}$ which avoids these extreme cases.

In our analysis, it is convenient to have a single hyperparameter regulating the support of $G_{0}$. For a given $\lambda_{\max }>0$, we find numerically the values of $a_{0}$ and $b_{0}$ which minimize the Kullback-Leibler divergence between $G_{0}$ and a uniform distribution on the interval $\left[0, \lambda_{\max }\right]$. This Kullback-Leibler divergence can be computed explicitly as

$$
-\log \lambda_{\max }-a_{0} \log b_{0}+\log \Gamma\left(a_{0}\right)-\left(a_{0}-1\right)\left(\log \lambda_{\max }-1\right)+\frac{b_{0} \lambda_{\max }}{2} .
$$

In this new parameterization, our goal is to make a sensible choice for $\lambda_{\max }$.

Our proposal to choose $\lambda_{\max }$ goes as follows. We fix some value $0 \leq \sigma<1$ for the discount parameter and choose an integer $k_{0}$ as the prior expectation of the number of clusters $K$, which, using the results at the end of Section 4.2, can be computed explicitly as

$$
\mathrm{E}[K]= \begin{cases}\tau \times(\psi(\tau+T-p)-\psi(\tau)) & \text { if } \quad \sigma=0 \\ \left((\tau+\sigma)_{T-p} /\left(\sigma \times(\tau+1)_{T-p-1}\right)\right)-\tau / \sigma & \text { if }\end{cases}
$$

in which $\psi(x)$ is the digamma function (see Pitman et al. [2002] for a derivation of this result). Next, we find the value of the concentration parameter $\tau$ by solving $\mathrm{E}[K]=k_{0} \mathrm{nu}-$ merically. After this, for each $\lambda_{\max }$ in a grid of values, we run the Gibbs sampler and compute the posterior expectation of the number of clusters $\mathrm{E}[K \mid y]$. Finally, in the corresponding graph, we look for the value of $\lambda_{\max }$ located at the "elbow" of the curve, that is, the value of $\lambda_{\max }$ at which the values of $\mathrm{E}[K \mid y]$ level off.

As an explicit example of this graphical criterion in action, we used the functional form of a first-order model with thinning parameter $\alpha=0.15$ to simulate a time series of length $T=1000$, for which the distribution of the innovations is a symmetric mixture of three Poisson distributions with parameters 1, 8, and 15. Figure 4.2 shows the formations of the elbows for two values of the discount parameter: $\sigma=0.5$ and $\sigma=0.75$.

For the simulated time series, Figures 4.3, 4.4, 4.5, and 4.6 display the behavior of the posterior distributions obtained using the elbow method for $\left(k_{0}, \sigma\right) \in\{4,10,16,30\} \times$ $\{0,0.25,0.5,0.75\}$. These figures make the relation between the choice of the value of the discount parameter $\sigma$ and the achieved robustness of the posterior distribution quite explicit: as we increase the value of the discount parameter $\sigma$, the posterior becomes insensitive to the choice of $k_{0}$. In particular, for $\sigma=0.75$, the posterior mode is always near 3 , which is the number of components used in the distribution of the innovations of the simulated time series. 

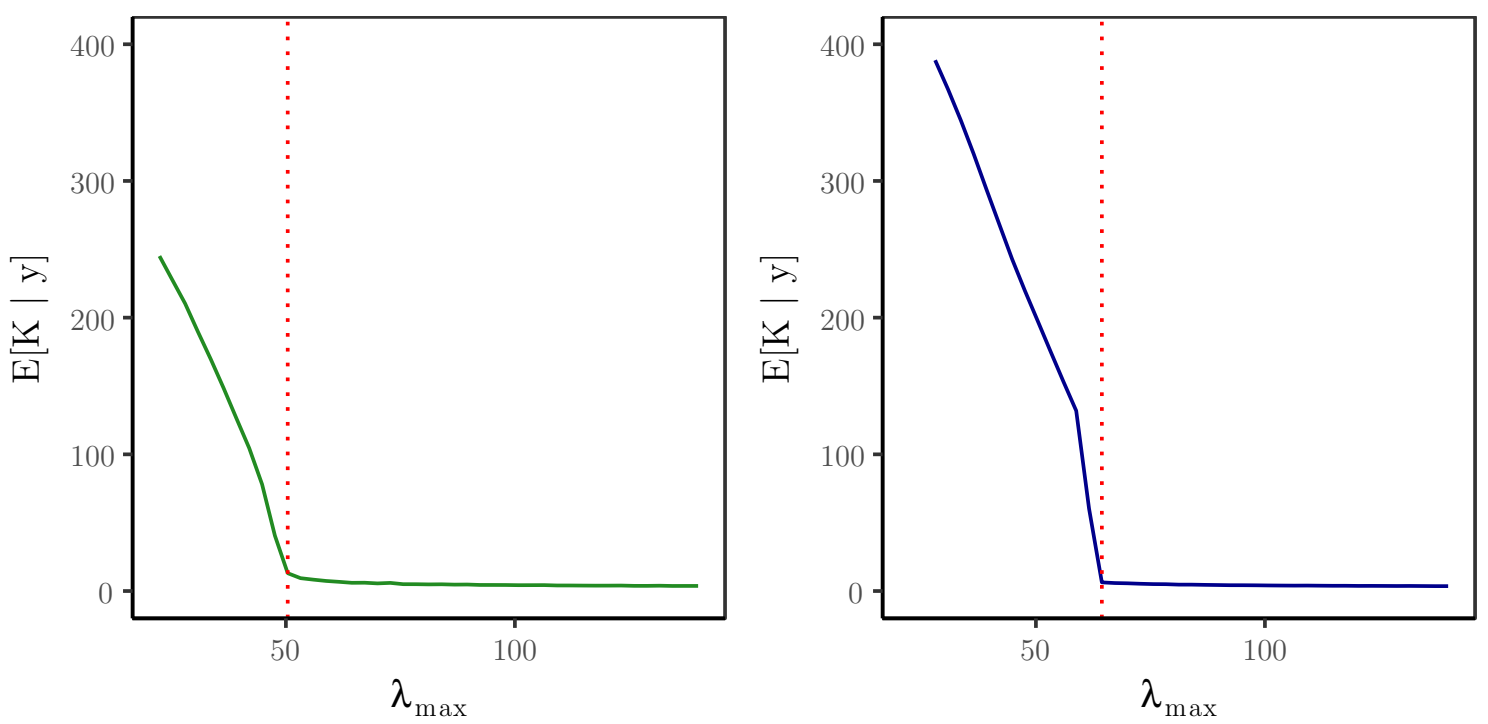

Figure 4.2: Formation of the elbows for $\sigma=0.5$ (left) and $\sigma=0.75$ (right). The red dotted lines indicate the chosen values of $\lambda_{\max }$.

\begin{tabular}{cccc}
\hline \hline & \multicolumn{3}{c}{ True } \\
\hline \hline Predicted & 1 & 2 & 3 \\
1 & 297 & 32 & 0 \\
2 & 11 & 217 & 42 \\
3 & 0 & 84 & 316 \\
\hline \hline
\end{tabular}

Table 4.1: Confusion matrix for the cluster assignments.

Once we understand the influence of the prior parameters on the robustness of the posterior distribution, an interesting question is how to get a point estimate of the distribution of clusters, in the sense that each $\lambda_{t}$, for $t=p+1, \ldots, T$, would be assigned to one of the available clusters. From the Gibbs sampler, it is easy to obtain an approximation for the probabilities $d_{r t}=\operatorname{Pr}\left\{\lambda_{r} \neq \lambda_{t} \mid y\right\}$, for $r, t=p+1, \ldots, T$. These probabilities define a dissimilarity matrix $D=\left(d_{r t}\right)$ among the innovation rates. Although $D$ is not a distance matrix, we can use it as a starting point to represent the innovation rates in a two dimensional Euclidean space using the technique of metric multidimensional scaling (see Friedman et al. [2009] for a general discussion). From this two dimensional representation, we build a dendrogram, which is appropriately cut in order to define three clusters, allowing us to assign a single cluster label to each innovation rate. Table 4.1 displays the confusion matrix of this assignment, showing that $83 \%$ of the innovations were grouped correctly in the clusters which correspond to the mixture components used to simulate the time series. A visualization of this difference between assigned and simulated clusters is given on Figure 4.7 . 
$\sigma=0$
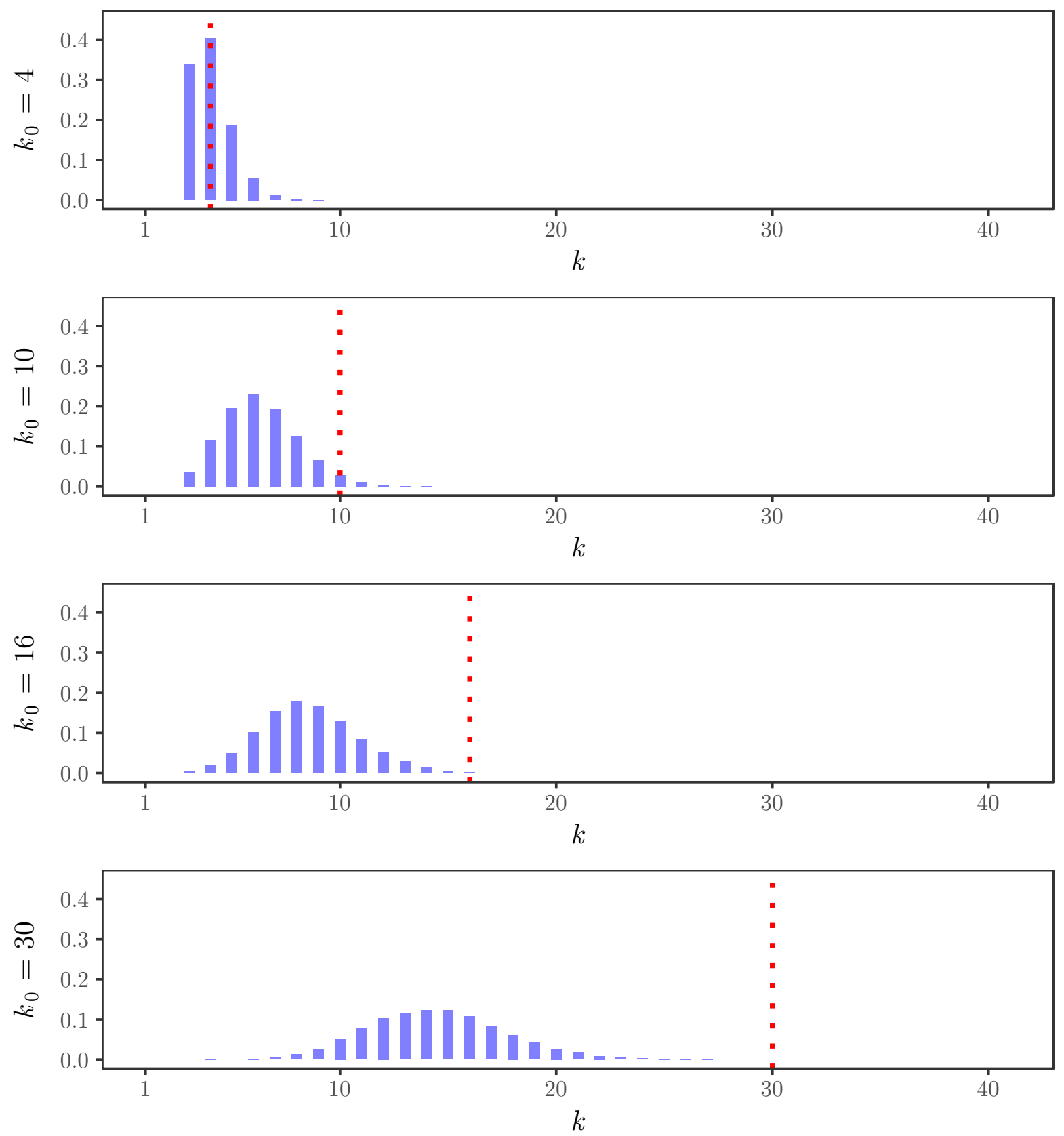

Figure 4.3: Posterior distributions of the number of clusters $K$ for the simulated time series with $\sigma=0$ and $k_{0}=4,10,16,30$. The red dotted lines indicate the value of $k_{0}$. 

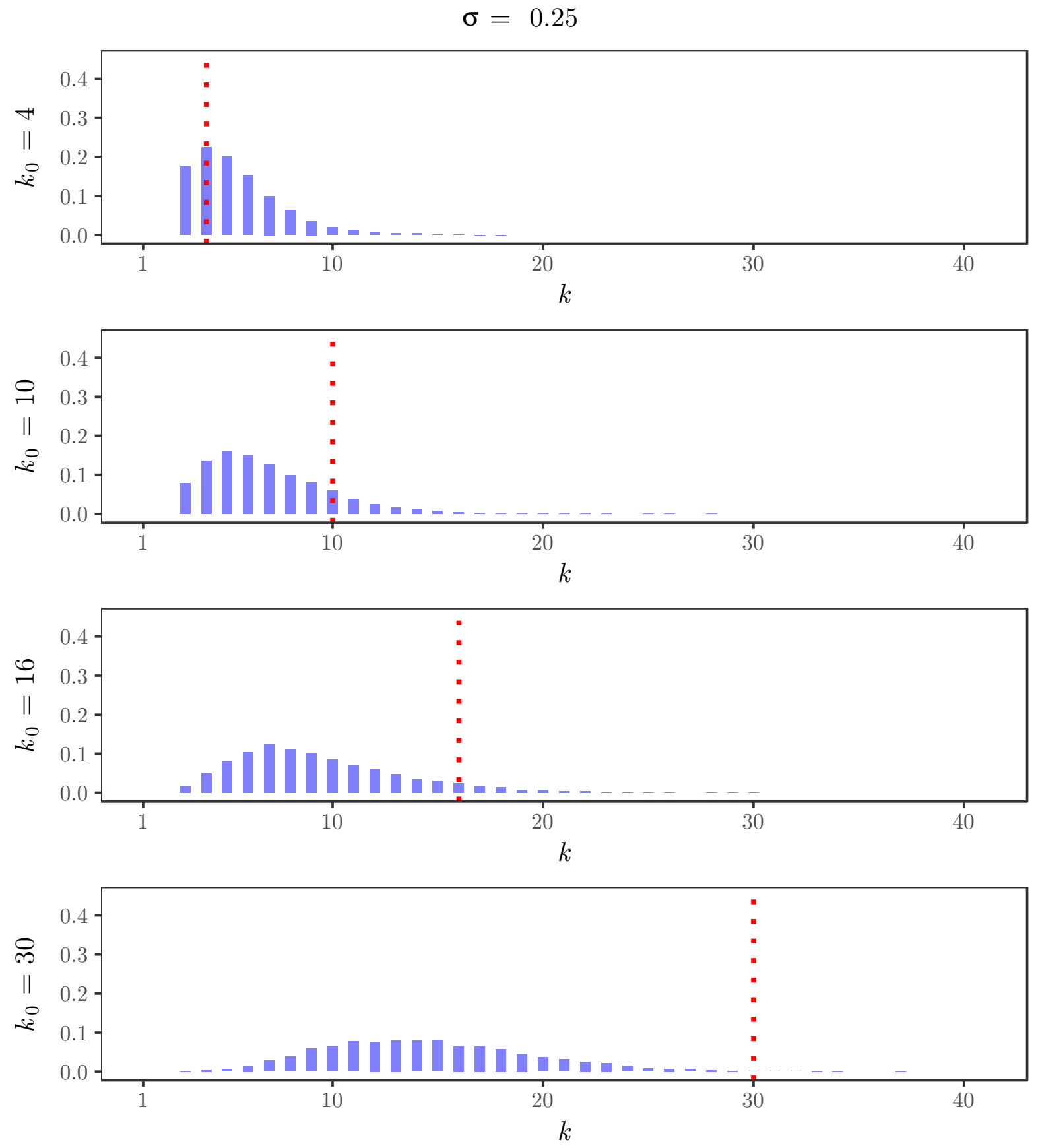

Figure 4.4: Posterior distributions of the number of clusters $K$ for the simulated time series with $\sigma=0.25$ and $k_{0}=4,10,16,30$. The red dotted lines indicate the value of $k_{0}$. 
$\sigma=0.5$
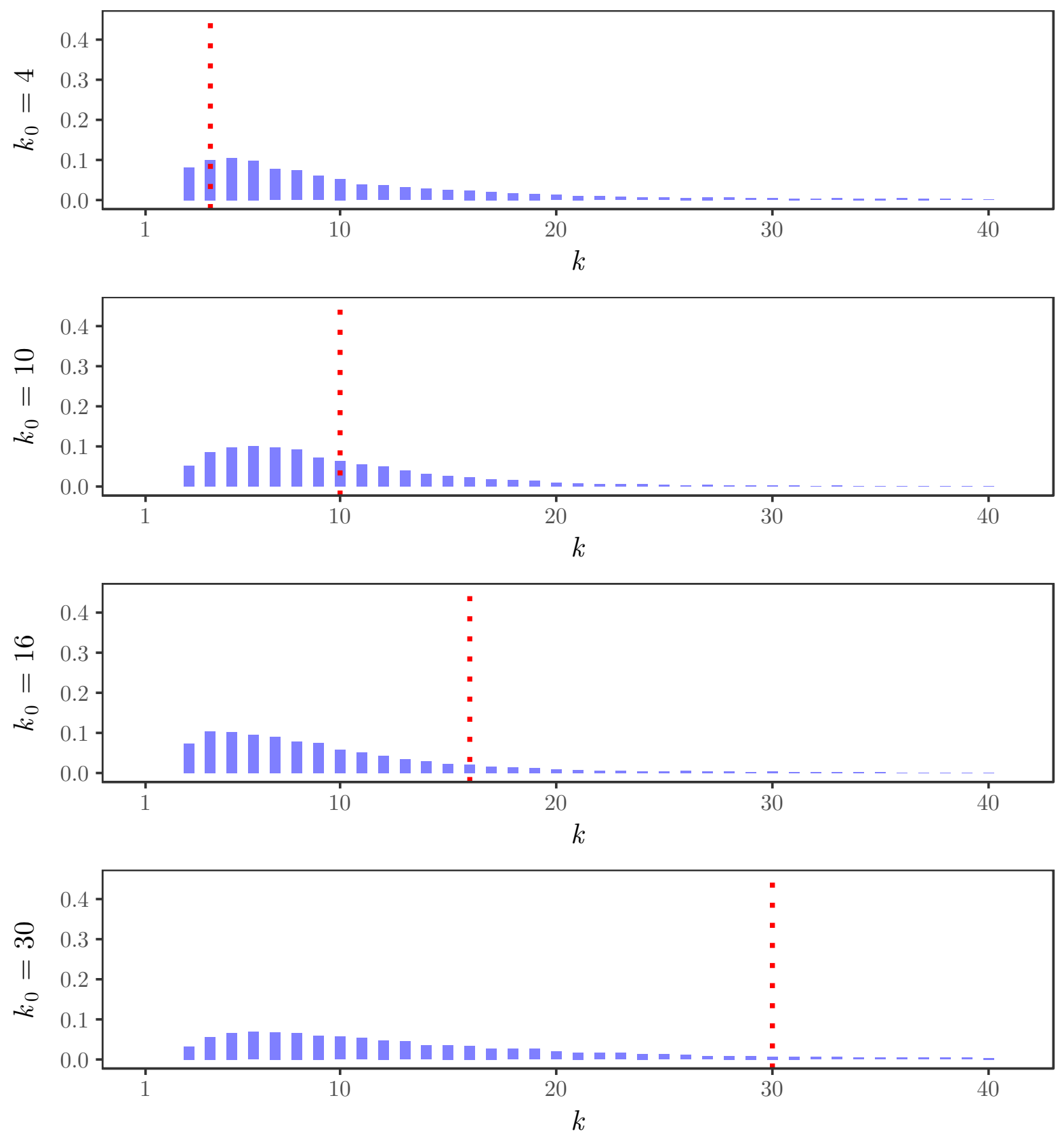

Figure 4.5: Posterior distributions of the number of clusters $K$ for the simulated time series with $\sigma=0.5$ and $k_{0}=4,10,16,30$. The red dotted lines indicate the value of $k_{0}$. 

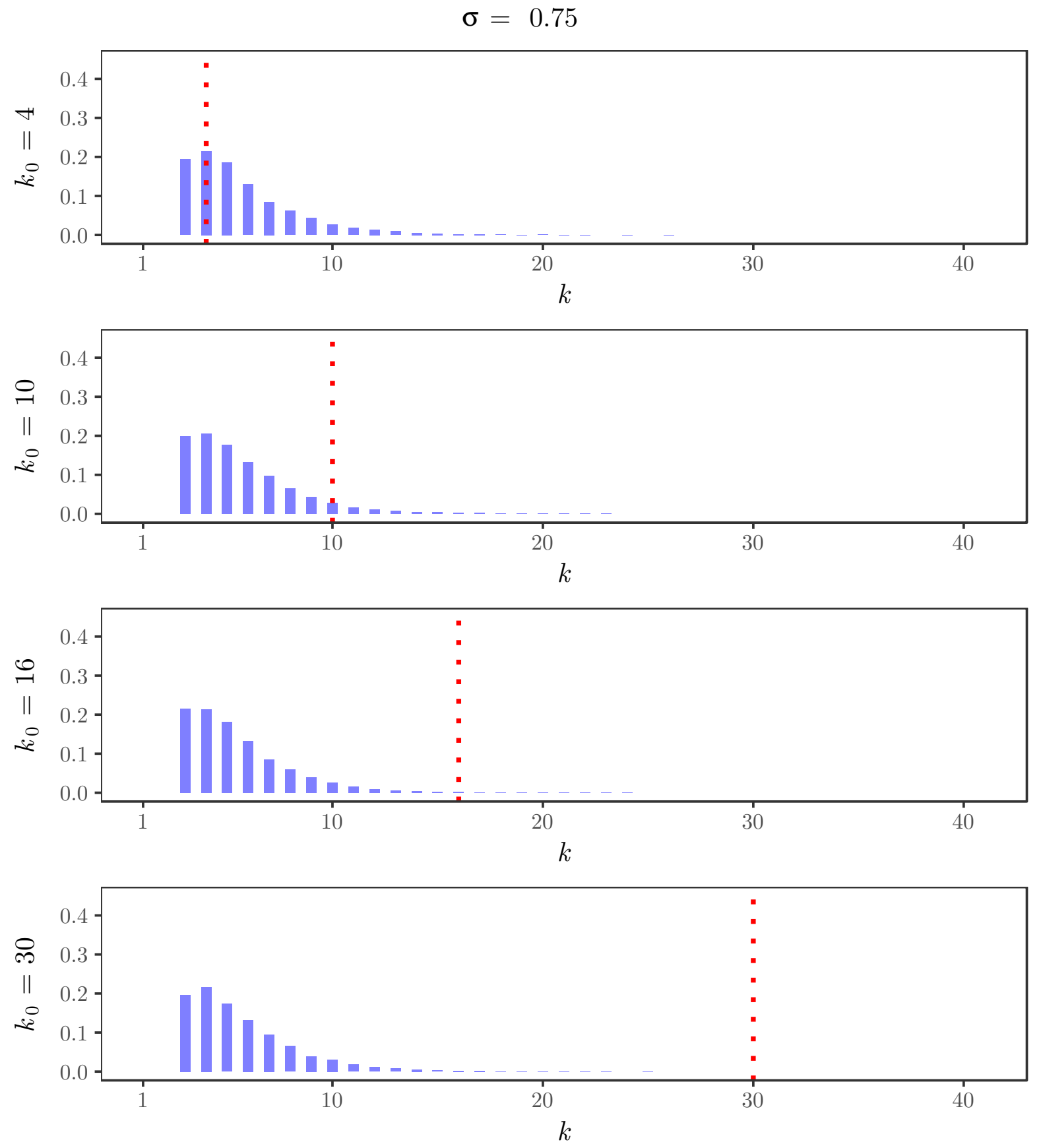

Figure 4.6: Posterior distributions of the number of clusters $K$ for the simulated time series with $\sigma=0.75$ and $k_{0}=4,10,16,30$. The red dotted lines indicate the value of $k_{0}$. 


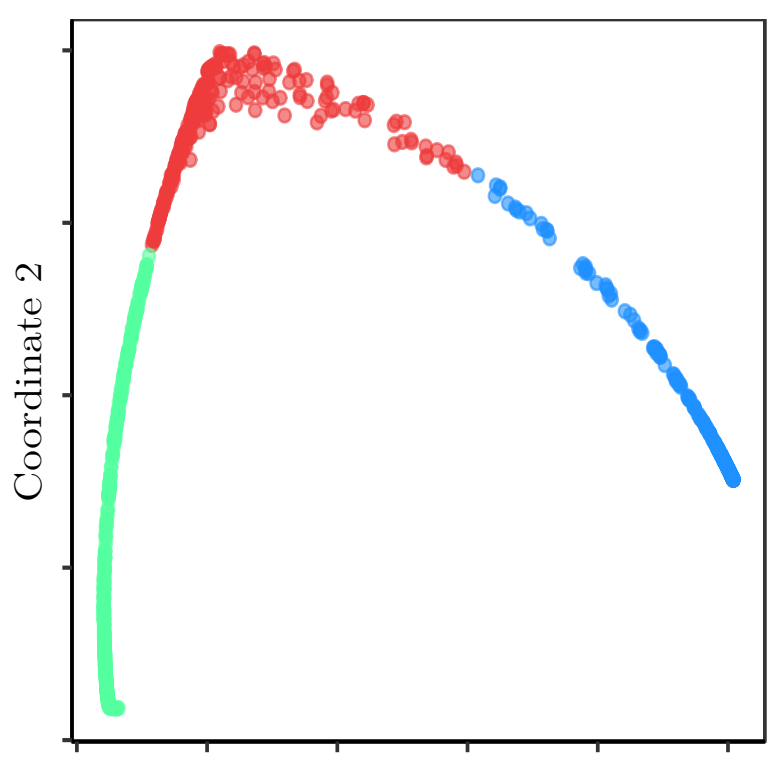

Coordinate 1

Cluster $\bullet 1 \circ 2 \circ 3$

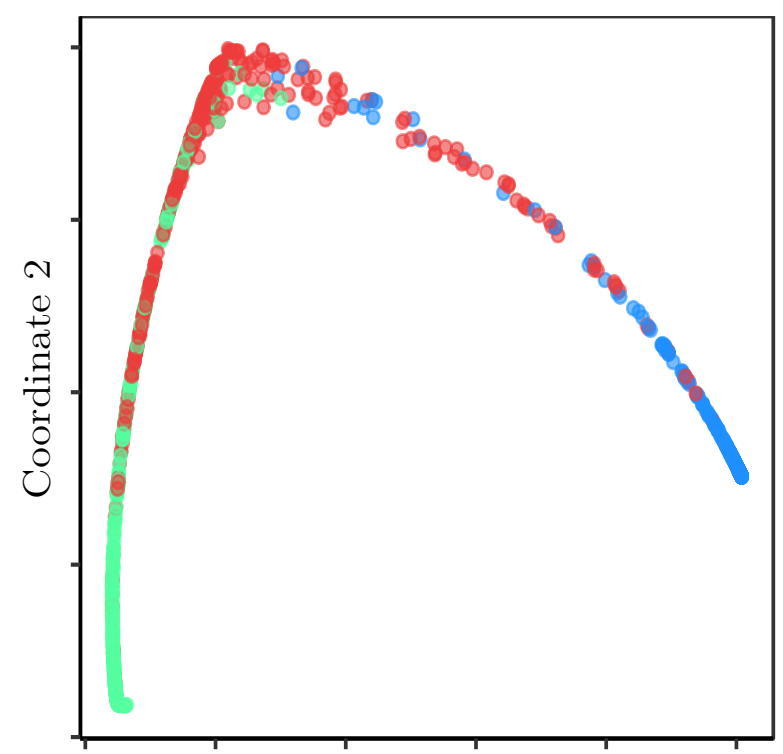

Coordinate 1

$\lambda \circ 1 \circ 8 \circ 15$

Figure 4.7: Swallow tails obtained by multidimensional scaling. The left and right figures show the assigned and simulated cluster labels, respectively

\subsection{Bayesian forecasting}

Similar to the DP-INAR(1) model, the Gibbs sampler described in the previous section yields, marginally, a sample $\left\{\alpha_{1}^{(n)}, \ldots, \alpha_{p}^{(n)}, \lambda^{(n)}\right\}_{n=1}^{N}$ from the posterior distribution. As a consequence, uncertainty about future counts is represented by the $h$-steps-ahead posterior predictive distribution

$$
Y_{T+h} \mid Y_{1}=y_{1}, \ldots, Y_{T}=y_{T},
$$

By using the law of total probability, the product rule, and simplifying the conditional independences in the model, we can write the posterior predictive probability function as

$$
\begin{aligned}
p\left(y_{T+h} \mid y_{1}, \ldots, y_{T}\right)=\int & p\left(y_{T+h} \mid y_{T}, \alpha_{1}, \ldots, \alpha_{p}, \lambda_{T+1}, \ldots, \lambda_{T+h}\right) \\
& \times \prod_{i=1}^{h} p\left(\lambda_{T+i} \mid \lambda_{2}, \ldots, \lambda_{T+i-1}\right) \\
& \times p\left(\alpha_{1}, \ldots, \alpha_{p}, \lambda_{2}, \ldots, \lambda_{T} \mid y_{1}, \ldots, y_{T}\right) d \alpha_{1} \ldots d \alpha_{p} d \lambda_{2} \ldots d \lambda_{T+h} .
\end{aligned}
$$


Notice that for $h=1$ it is possible to find the moment generating function

$$
\begin{aligned}
M_{Y_{t+1} \mid Y_{t}, \ldots Y_{t-p+1}}(s) & =\mathrm{E}\left[e^{s Y_{t+1}} \mid Y_{t}, \ldots, Y_{t-p+1}\right]=\mathrm{E}\left[e^{s\left(\alpha_{1} \circ Y_{t}+\cdots+\alpha_{p} \circ Y_{t-p+1}+Z_{t+1}\right)} \mid Y_{t}, \ldots, Y_{t-p+1}\right] \\
& =\prod_{i=1}^{p}\left(\alpha_{i} e^{s}+\left(1-\alpha_{i}\right)\right)^{Y_{t}-i+1} \exp \left(\lambda_{t+1}\left(e^{s}-1\right)\right)
\end{aligned}
$$

which is the product of the generating functions of $p$ Binomial random variables with parameters $Y_{t-i+1}$ and $\alpha_{i}, i=1, \ldots, p$ and a Poisson $\left(\lambda_{t+1}\right)$ random variables. On the other hand, since for a general $h$ the corresponding generating moment function involves a high number of convolutions, we approximate the probability function simulating the future sequentially, generating synthetic counts

$$
\begin{aligned}
y_{T+1}^{(n)} & =\alpha_{1}^{(n)} \circ y_{T}+\cdots+\alpha_{p}^{(n)} \circ y_{T-p+1}+z_{T+1}^{(n)}, \\
& \vdots \\
y_{T+h}^{(n)} & =\alpha_{1}^{(n)} \circ y_{T+h-1}^{(n)}+\cdots+\alpha_{p}^{(n)} \circ y_{T+h-p+1}+z_{T+h}^{(n)},
\end{aligned}
$$

for $n=1, \ldots, N$, where the future innovations may be obtained by the generalized PólyaBlackwell-Macqueen rule, as described in Section 3.6. After obtaining the pointwise prediction, we perform the cross-validation procedure. In the next section, we compare the performance of the PY-INAR $(p)$ model for time series of earthquake occurrences.

\subsection{Earthquake count data}

In this section, we analyze a time series of yearly worldwide earthquakes events of substantial magnitude (equal or greater than 7 points on the Richter scale) from 1900 to 2018 (http://www.usgs.gov/natural-hazards/earthquake-hazards/earthquakes).

In this section, we analyze a time series of earthquakes occurrences worldwide from 1900 to 2018 (per year). The counts corresponds to earthquakes with magnitude equal or greater than 7 points on the Richter scale. Figure 4.8 shows the time series plot of the corresponding time series which contains a substantial overdispersion, with mean 19.07 and variance 48.94. We trained the PY-INAR $(p)$ and $\operatorname{INAR}(p)$ models for $p=1,2,3$.

First, we will show the posterior results for the PY-INAR(3). Figure 4.10 shows the formation of the elbows for $\sigma=0.75$. The red dot indicates the chosen value for $\lambda_{\max }=60$.

Moreover, Figure 4.9 gives the posterior distributions of $\alpha_{1}, \alpha_{2}$ and $\alpha_{3}$, whose posterior 


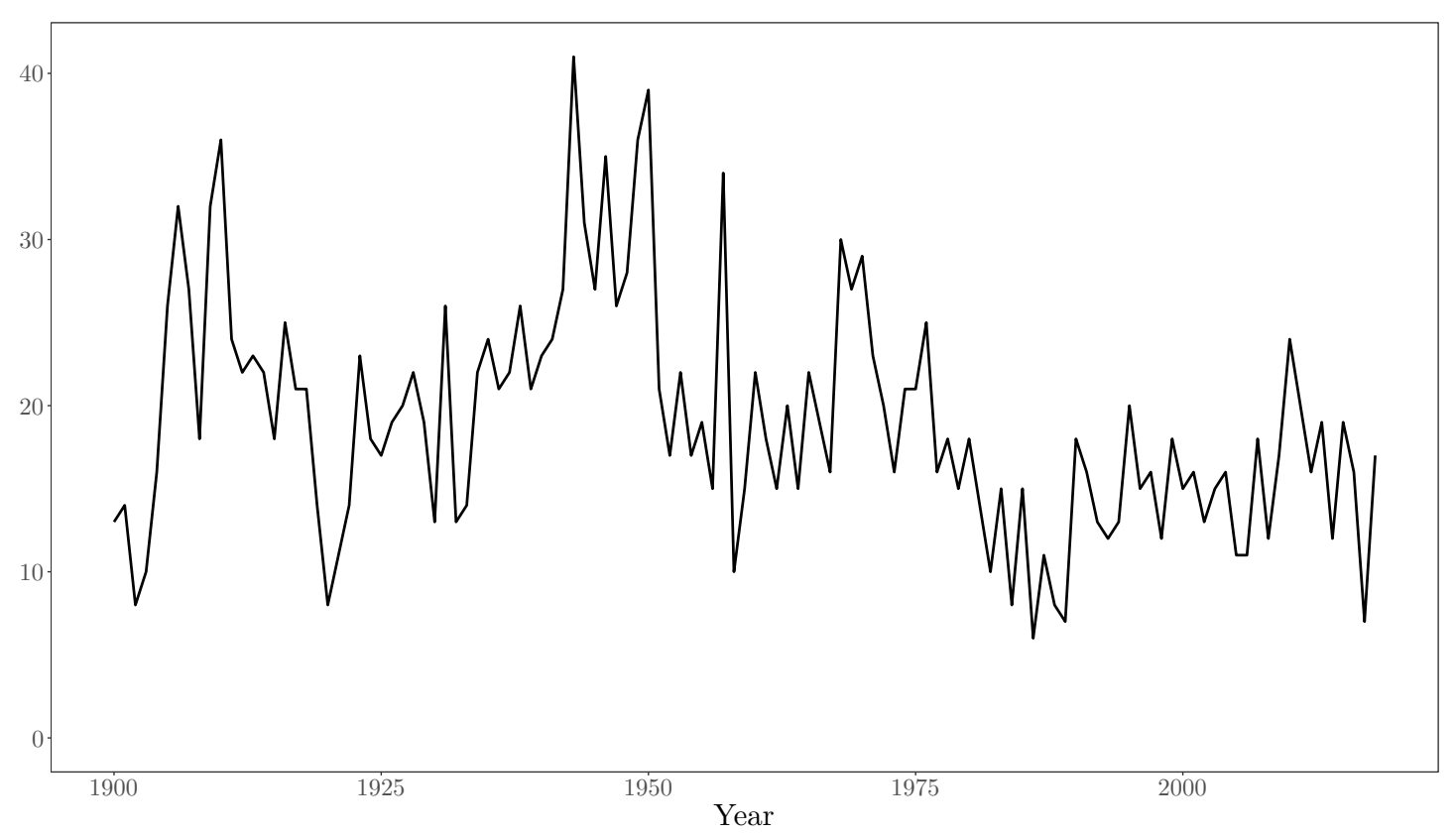

Figure 4.8: Worldwide earthquake events from 1900 to 2018 (per year).

means are equal to $0.27,0.14,0.15$, respectively. Also the chains in Figure 4.11 indicate that mixing is achieved by the proposed Gibbs sampler.
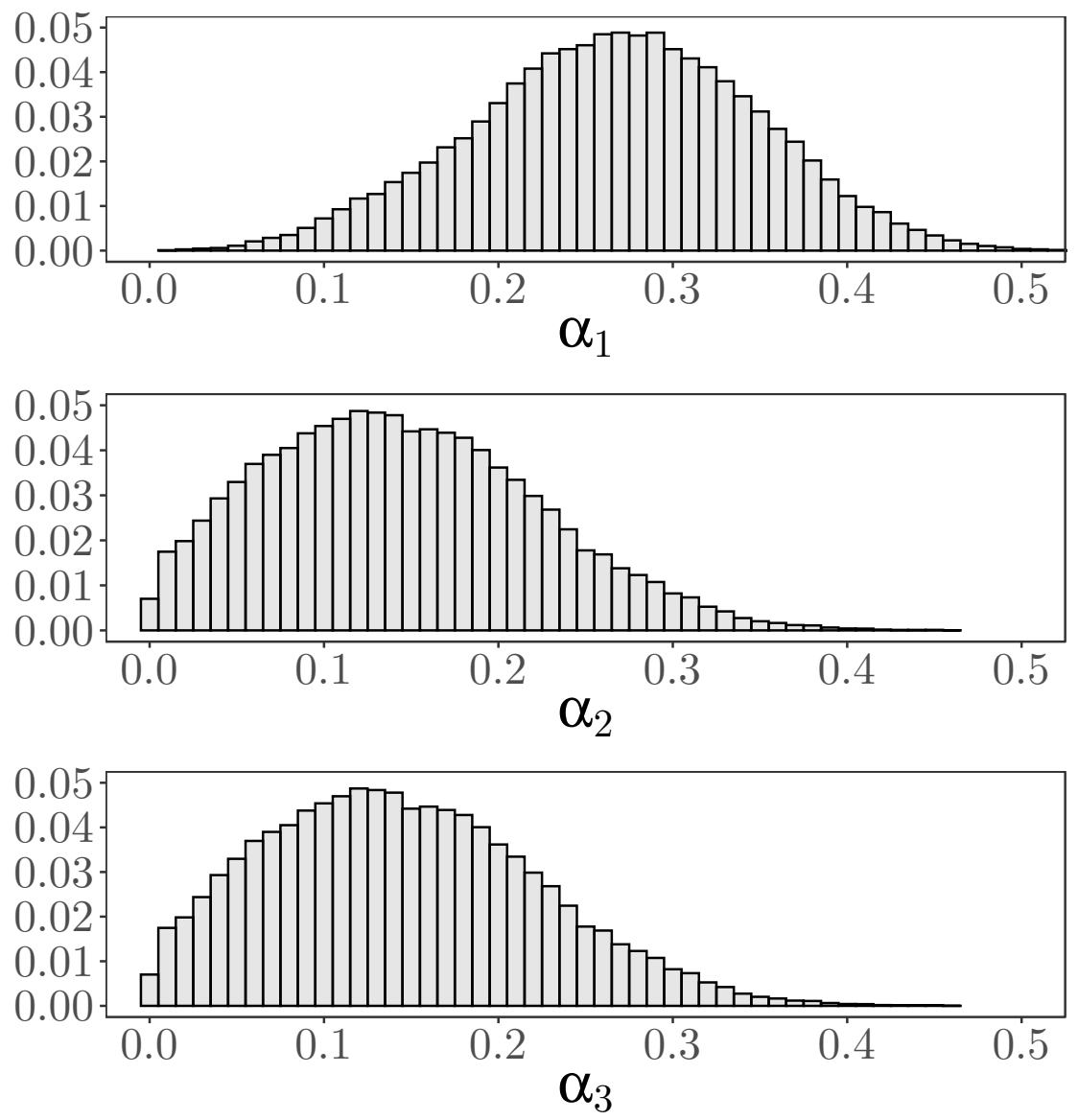

Figure 4.9: Marginal posterior distributions of the PY-INAR(3) model parameters $\alpha_{1}, \alpha_{2}$ and $\alpha_{3}$ for the earthquake data set. 


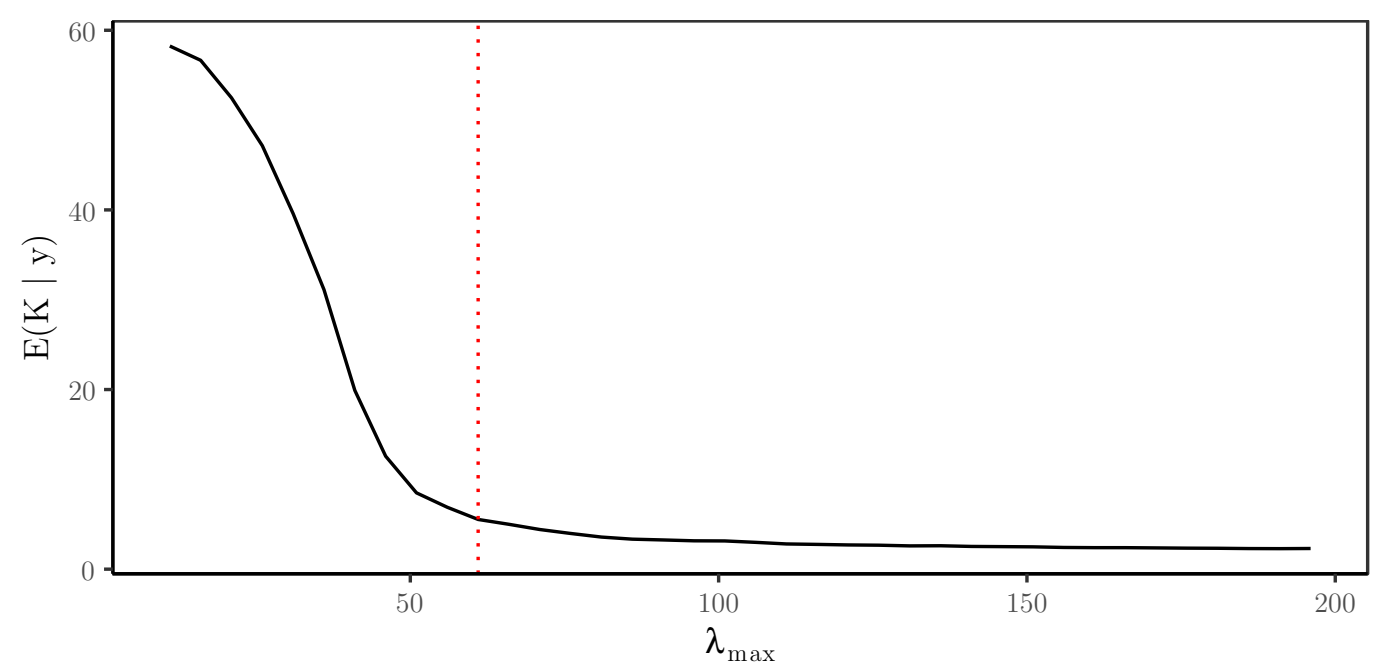

Figure 4.10: Elbow for the earthquake data set with $\sigma=0.75$ and $k_{0}=30$.
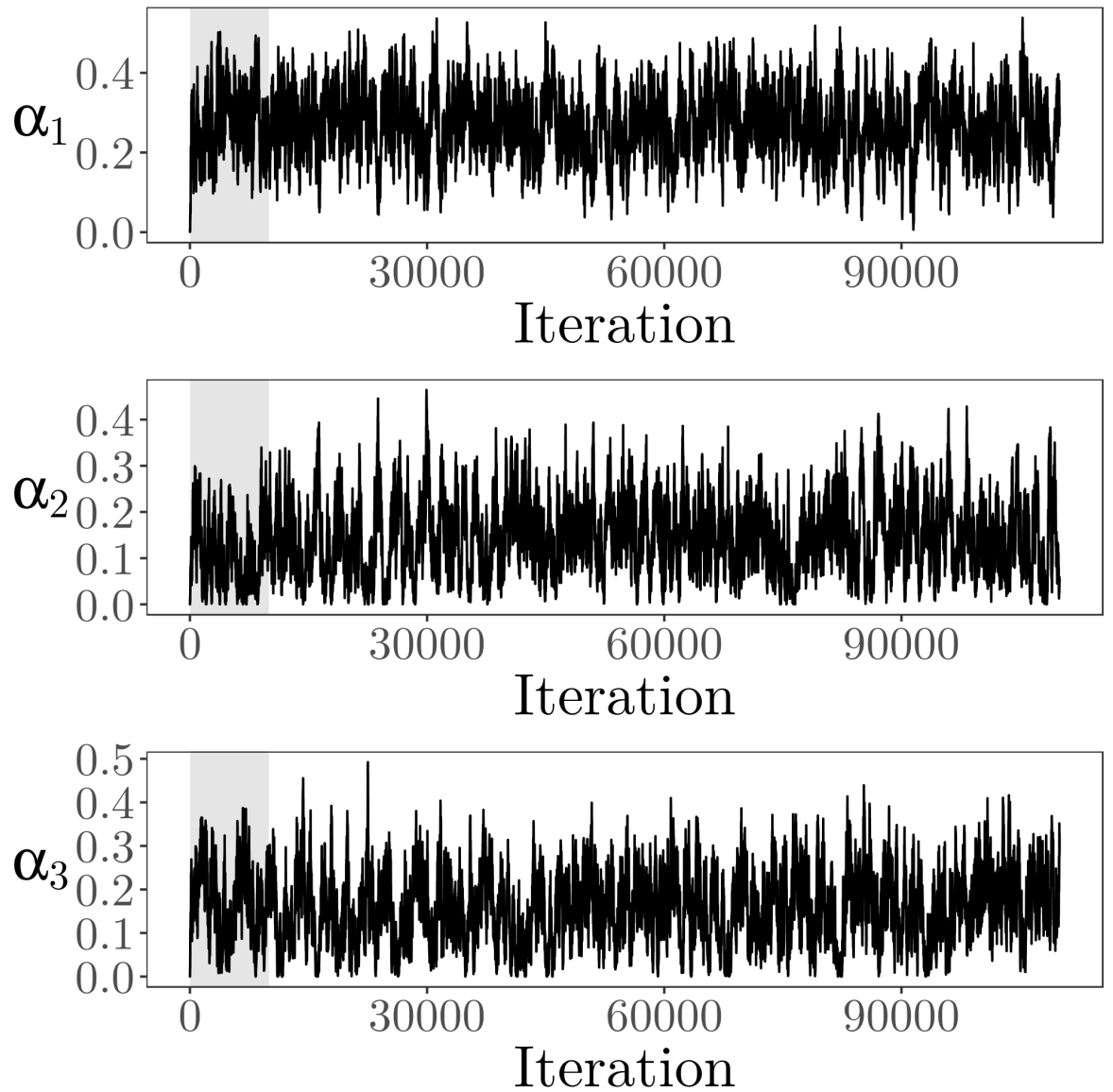

Figure 4.11: Markov chains associated with the PY-INAR(3) model marginal posterior distributions of parameters $\alpha_{1}, \alpha_{2}, \alpha_{3}$ for the earthquake data set. The gray rectangles indicate the burn-in periods. 
We perform the cross-validation procedure 3.6 for both the $\operatorname{INAR}(p)$ and $\operatorname{PY} \operatorname{INAR}(p)$ models, for $p \leq 3$. Using this cross-validation procedure, we trained the $\operatorname{INAR}(p)$ and the $\operatorname{PY} \operatorname{INAR}(p)$ models with orders $p=1,2$, and 3 , and made one-step-ahead predictions. Table 4.2 shows the out-of-sample mean absolute errors (MAE) for the $\operatorname{INAR}(p)$ and the PY-INAR $(p)$ models. In this table, the MAE's are computed predicting the counts for the last 36 months. For the three model orders, the PY-INAR $(p)$ model yields a smaller MAE than the original $\operatorname{INAR}(p)$ model.

\begin{tabular}{cccccccc}
\hline & INAR & AdINAR & DP-INAR & PY-INAR & $\Delta_{\text {Ad-INAR }}$ & $\Delta_{\text {DP-INAR }}$ & $\Delta_{\text {PY-INAR }}$ \\
\hline$p=1$ & 3.861 & 3.917 & 3.889 & 3.583 & -0.015 & 0.007 & -0.072 \\
$p=2$ & 3.583 & 3.583 & 3.444 & 3.417 & 0.000 & -0.039 & -0.046 \\
$p=3$ & 3.972 & 3.917 & 3.472 & 3.305 & -0.014 & -0.126 & -0.202 \\
\hline
\end{tabular}

Table 4.2: Out-of-sample MAE's for the INAR(p) and the PY-INAR(p) models, with orders $p=$ 1,2 , and 3. The last column shows the relative variations of the MAE's for the PY-INAR(p) models with respect to the corresponding MAE's for the INAR(p) models. 


\section{Chapter 5}

\section{Conclusions}

\subsection{Final considerations}

In this thesis, we proposed Bayesian generalizations of the INAR(1) model of [McKenzie, 1985] and [Al-Osh and Alzaid, 1988]. Our starting point was the AdINAR(1) model which accounts for overdispersion in the time series using a Geometric-Poisson mixture as the marginal distribution of the innovation process. Second, the DP-INAR(1) model is capable of learning a latent pattern of heterogeneity in the distribution of the innovation rates by means of a Dirichlet process placed at the top of the model hierarchy. Under this approach, we showed a proposition that allows us to obtain the predictive distribution of future steps of the process. Finally, we generalized the autoregressive order by using a Pitman-Yor process in the distribution of the innovation rates, which entails robust results for specific choices of the process hyperparameters. For all models, we devised data augmentation schemes from which we derive full conditional distributions in simple analytical forms. Simulations of the posterior distributions through Gibbs sampling, and a predictive cross-validation procedure, give evidence of good forecasting performance in the analysis of two datasets. The first one comprises times series of burglary events in Pittsburgh, USA, while the second dataset shows Earthquake occurences worldwide. In both applications, the proposed models outperformed the benchmark models for discrete time series. Furthermore, we developed an open source $\mathrm{R}$ package with the proposed models for training and prediction, which is available at: https://github.com/heltongraziadei/BayesINAR. 


\subsection{Suggestions for future research}

Several extensions of our models could be investigated, such as:

1. Generalize the proposed models for multivariate time series through Hierarchical Dirichlet Processes as well as investigating the sensitivity of hyperparameters under this approach.

2. Incorporate covariates in the model by using a link function on the innovation rates in both the parametric and semi-parametric models.

3. Investigate alternatives to incorporate trend and seasonality into the model hierarchy.

4. Parallelize the cross-validation procedure to accelerate the model performance assessment.

5. Investigate Bayesian methods to learn the autoregressive order. 


\section{Appendix A}

\section{BayesINAR: An R package for Bayesian modeling time series of counts}

The BayesINAR package was developed for training and predicting from the INAR, AdINAR, DP-INAR and PY-INAR models. In this appendix, we give a brief summary on how to install and use this package for analyzing time series of counts. The full package is available at http://www.github.com/heltongraziadei/BayesINAR. Essentially, we coded the Gibbs samplers proposed in Chapters 2, 3 and 4 with the help of the Rcpp package [Eddelbuettel, 2013] as a means to speed up computations, mainly the full conditionals calculations.

Initially, it is necessary to install the package by loading the devtools package and using the command install_github to download the repository from Github, as follows:

1 library (devtools)

2 install_github ("heltongraziadei/BayesINAR")

Listing A.1: Installing the BayesINAR package

Now that the BayesINAR package is successfully installed, we illustrate how to use it for a specific time series of burglary cases of Pittsburgh, USA, which was explored in Section 3.7.

1 library (Bayes INAR)

2 data(Pittsburgh)

3 y $<-$ Pittsburgh\$Area_58 
Listing A.2: Loading the package and the time series of burglary cases

We use function inar to train the INAR model, specifying the vector of the time series and the autoregressive order of the corresponding model. The functions returns an object of the class inar. After we have this object, we may call the summary and the predictive distribution for the $h$-step-ahead. The default hyperparameters of the model corresponds to weak informative prior choices, but it may be specified by the user as well, through the list model. The parameters of the inar function are:

- time_series: corresponding time series of counts.

- $\mathrm{p}$ : autoregressive order of the model

- prior: a list with the following arguments:

- a_alpha: Hyperparameters for the vector of thinning parameters.

- a_lambda and b_lambda: Hyperparameters of the Gamma prior for the homogeneous Poisson innovation rate.

- burn_in: Number of iterations for the "burn-in" period which are discarded in the chain.

- chain_length: Number of iterations of the chain.

- verbose: If TRUE log information is provided.

Consequently, the function inar is used do train the corresponding INAR model, creating an object of class inar with methods summary and predict, as follows:

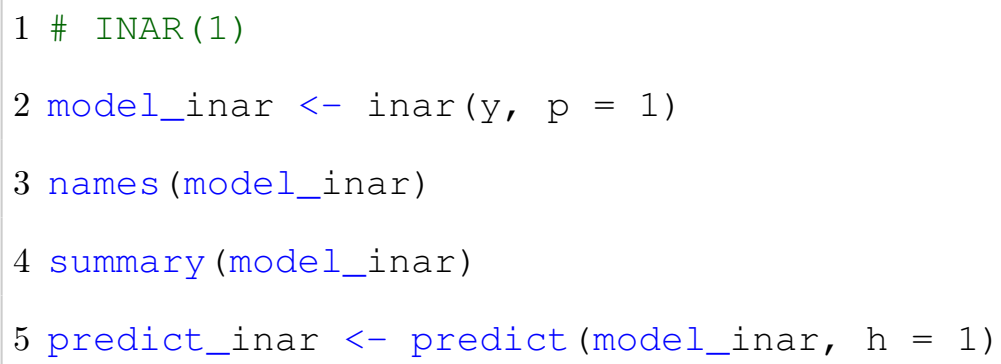


Similarly, the AdINAR, DP-INAR and PY-INAR models are trained through the adinar, dpinar and pyinar functions, respectively. The only difference in the parameters of these functions is in the prior list with varies depending on which model is trained. The complete list of prior hyperparameters is available in the package documentation.

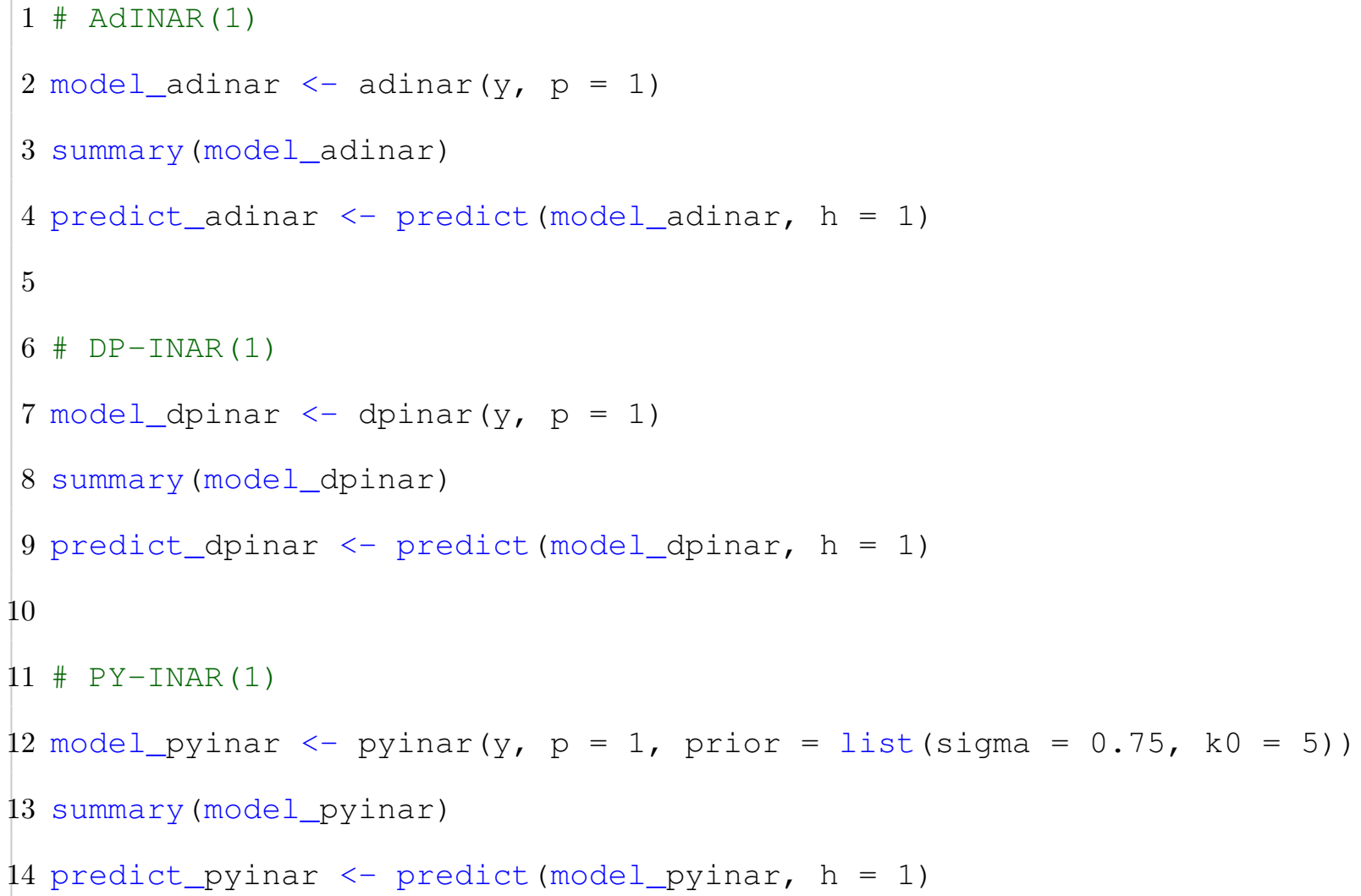

Finally, the cross-validation procedure described in Figure 3.2 may be performed with the help of the function cross_validate. The parameters of this function is the time series of counts, the autoregressive order and the model desired to cross-validate. It returns the predictions on the test set and the out-of-sample mean absolute errors (MAEs).

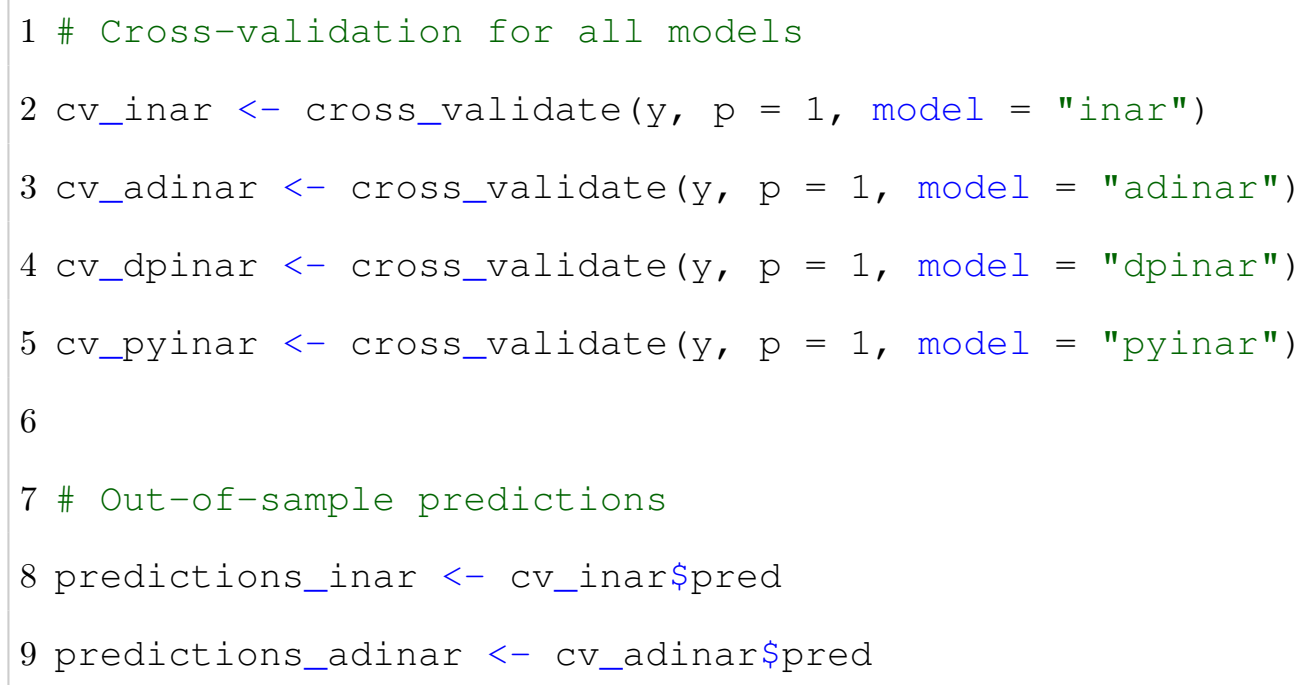




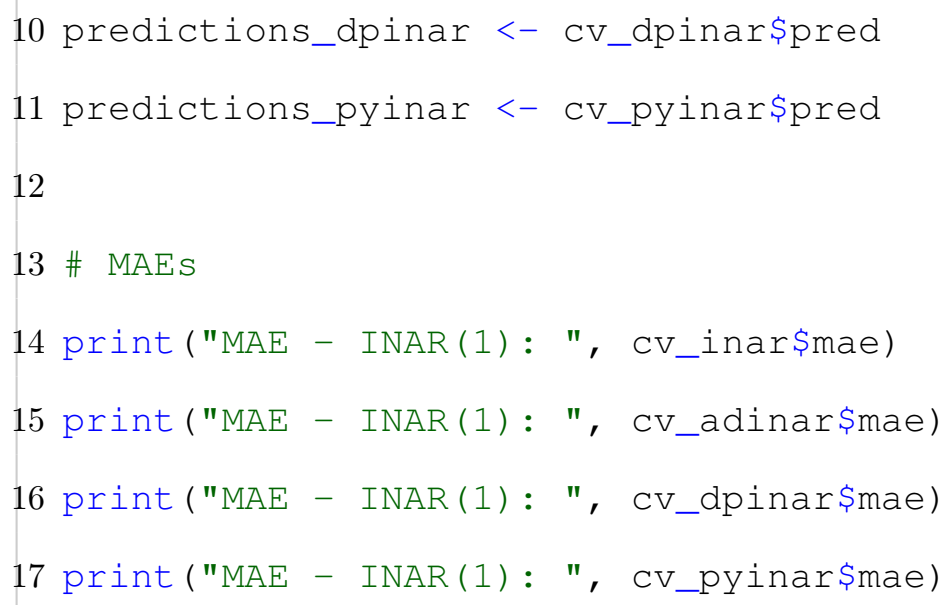

Listing A.4: Cross-validation procedure 


\section{References}

Al-Osh, M. and Alzaid, A. (1988). First-order integer-valued autoregressive (INAR(1)) process: distributional and regression properties. Statistica Neerlandica, 42:53-61. 1, 5, 15,57

Antoniak, C. (1974). Mixtures of Dirichlet processes with applications to Bayesian nonparametric problems. The Annals of Statistics, 2(6):1152-1174. 19

Blackwell, D. and MacQueen, J. (1973). Ferguson distributions via Pólya urn schemes. The Annals of Statistics, 1(2):353-355. 19

Bourguignon, M. and Weiß, C. H. (2017). An inar (1) process for modeling count time series with equidispersion, underdispersion and overdispersion. Test, 26(4):847-868. 1

Canale, A. and Prünster, I. (2017). Robustifying bayesian nonparametric mixtures for count data. Biometrics, 73(1):174-184. 3, 44

Davis, R. A., Holan, S. H., Lund, R., and Ravishanker, N. (2016). Handbook of discretevalued time series. CRC Press. 2

Dion, J., Gauthier, G., and Latour, A. (1995). Branching processes with immigration and integer-valued time series. Serdica Mathematical Journal, 21(2):123-136. 38

Dorazio, R. (2009). On selecting a prior for the precision parameter of Dirichlet process mixture models. Journal of Statistical Planning and Inference, 139(9):3384-3390. 3, 23

Du, J.-G. and Li, Y. (1991). The integer-valued autoregressive (INAR(p)) model. Journal of Time Series Analysis, 12:129-142. 37, 42

Eddelbuettel, D. (2013). Seamless $R$ and $C++$ integration with Rcpp. Springer. 59 
Escobar, M. and West, M. (1998). Computing nonparametric hierarchical models. In Dey, D., Müller, P., and Sinha, D., editors, Practical nonparametric and semiparametric Bayesian statistics, chapter 1, pages 1-22. Springer-Verlag. 22

Ferguson, T. (1973). A Bayesian analysis of some nonparametric problems. The Annals of Statistics, 1(2):209-230. 2, 18, 40

Freeland, K. (1998). Statistical analysis of discrete time series with application to the analysis of workers' compensation data. PhD thesis, University of British Columbia, Vancouver. 1

Friedman, J., Hastie, T., and Tibshirani, R. (2009). The elements of statistical learning. Springer Series in Statistics. 46

Gamerman, D. and Lopes, H. (2006). Markov chain Monte Carlo: stochastic simulation for Bayesian inference. Chapman \& Hall / CRC. 11, 22, 43

Hamilton, J. (1994). Time series analysis, volume 2. Princeton university Press. 42

Hjort, N., Holmes, C., Müller, P., and Walker, S. (2010). Bayesian nonparametrics, volume 28. Cambridge University Press. 40

Jazi, M., Jones, G., and Lai, C.-D. (2012). Integer valued ar (1) with geometric innovations. Journal of the Iranian Statistical Society, 11(2):173-190. 1

Jordan, M. (2004). Graphical models. Statistical Science, 19(1):140-155. 9, 20

Lijoi, A., Mena, R. H., and Prünster, I. (2007). Bayesian nonparametric estimation of the probability of discovering new species. Biometrika, 94(4):769-786. 41

McKenzie, E. (1985). Some simple models for discrete variate time series. Journal of the American Water Resources Association, 21(4):645-650. 1, 5, 15, 57

Neal, P. and Kypraios, T. (2015). Exact bayesian inference via data augmentation. Statistics and Computing, 25(2):333-347. 2, 42

Pitman, J. et al. (2002). Combinatorial stochastic processes. Technical report, Technical Report 621, Dept. Statistics, UC Berkeley, 2002. Lecture notes. 40, 45 
Robert, C. and Casella, G. (2013). Monte Carlo statistical methods. Springer Science \& Business Media. 11

Schervish, M. J. (1995). Theory of statistics. Springer Series in Statistics. 40

Silva, I., Silva, M. E., Pereira, I., and Silva, N. (2005). Replicated INAR(1) processes. Methodology and Computing in applied Probability, 7(4):517-542. 1, 2

Silva, N., Pereira, I., and Silva, M. E. (2009). Forecasting in INAR(1) model. REVSTATStatistical Journal, 7(1):119-134. 1

Sudderth, E. B. (2006). Graphical models for visual object recognition and tracking. PhD thesis, Massachusetts Institute of Technology. 3

Tanner, M. and Wong, W. (1987). The calculation of posterior distributions by data augmentation. Journal of the American Statistical Association, 82(398):528-540. 9, 17, 39

Van Dyk, D. and Meng, X.-L. (2001). The art of data augmentation. Journal of Computational and Graphical Statistics, 10(1):1-50. 9, 39

Weiß, C. (2018). An introduction to discrete-valued time series. John Wiley \& Sons. 1, 2, 5

Weiß, C. H. (2008). Thinning operations for modeling time series of counts - - a survey. AStA Advances in Statistical Analysis, 92(3):319. 1

West, M. (1992). Hyperparameter estimation in Dirichlet process mixture models. Duke University ISDS discussion paper \#92-A03. 22 